\title{
Ka-band Single-ended 3-Stack Power Amplifier in 45-nm RF-SOI
}

by

\section{Minho Jung, B.ASc.}

A thesis submitted to the Faculty of Graduate and Postdoctoral Affairs in partial fulfillment of the requirements for the degree of

\section{Master of Applied Science}

in

\section{Electrical and Computer Engineering}

Ottawa-Carleton Institute for Electrical and Computer Engineering

Department of Electronics

Carleton University

Ottawa, Ontario, Canada

December 2019

Copyright (C) 2019

Minho Jung 


\section{Abstract}

With the allocation of Ka-band as one of the frequency bands for the upcoming $5 \mathrm{G} \mathrm{mmWave}$ wireless communication, it is anticipated that there will be a massive increase in the demand for cost-effective mmWave power amplifiers (PAs) that can potentially be integrated in the RF front-end modules (RF-FEMs) of billions of 5G handsets and small-cell radios.

RF-SOI (Silicon-On-Insulator) process has emerged as a strong contender to III-V processes (e.g., GaAs, InP, and $\mathrm{GaN}$ ) in terms of a candidate process for $5 \mathrm{G} \mathrm{mmWave} \mathrm{PAs.} \mathrm{RF-}$ SOI retains most of the advantages of the bulk CMOS (i.e., high integration capability, low power consumption, and evident economies of scale), yet also offers features that are tailored for RF/mmWave circuits (i.e., RF-dedicated devices, high-resistivity (HR) substrate, and back end of line (BEOL) metal stack that includes thick copper and aluminum layers). Furthermore, the floating-body partially depleted (PD) devices offered in the RF-SOI process facilitate transistor stacking, which allows the PA to achieve higher RF output power while overcoming the high knee voltage and low breakdown voltage limitations of the advanced node RF-SOI devices.

This thesis presents the design and implementation of a fully integrated $28 \mathrm{GHz} 3$-stack linear PA in 45-nm RF-SOI process. The research work focuses on the design and optimization of transistor cell layout, transistor stacking technique, and layout-driven PA design method - all of which are critical to achieving high RF performance at mmWave frequencies. The implemented PA operates from a 3.3V power supply, and under a continuous wave (CW) RF input, it delivers a saturated output power of $19.5 \mathrm{dBm}$. Furthermore, the PA achieves a peak power-added efficiency (PAE) and a power gain of $31.8 \%$ and $14.1 \mathrm{~dB}$, respectively. The PA chip occupies $0.66 \mathrm{~mm}^{2}(950 \mu \mathrm{m}$ by $700 \mu \mathrm{m})$ of die area, including the pads. 


\section{Acknowledgements}

I would like to take this opportunity to express my profound gratitude to my thesis supervisor, Dr. Rony Amaya, for his support and encouragement throughout my graduate studies.

I would also like to express my sincere appreciation to Skyworks Solutions Inc. and Greg Babcock for providing me with a research opportunity at Skyworks Solutions Inc. and chip fabrication. Furthermore, I would like to express my sincere thanks to Sanjeev Jain, Hassan Sarbishaei, John Nisbet, and Yasser Soliman for sharing the invaluable knowledge they have acquired over many years of working in the field. My research would not have been possible without their mentorship.

Many thanks to my Carleton friends, Paulyn, Behzad, Joseph, Rene, and Wenyu. I am very grateful for meeting you guys. Thanks to my Skyworks friends Kai and Nasen, for helping me out and making my days at Skyworks enjoyable.

Lastly, I would like to thank my parents and sisters for believing in me, supporting me, and motivating me during the course of this journey. 


\section{Table of Contents}

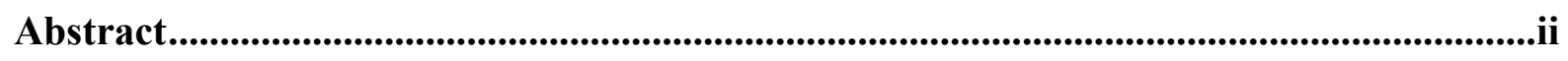

Acknowledgements ................................................................................................................ iii

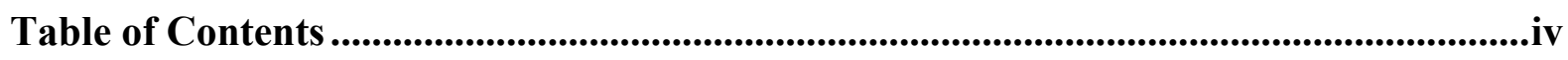

List of Tables ...................................................................................................................................vi

List of Figures.........................................................................................................................................vii

List of Acronyms and Symbols ........................................................................................................

Chapter 1: Introduction .............................................................................................................15

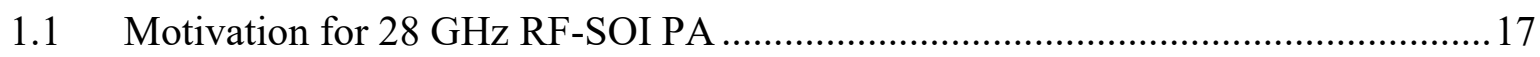

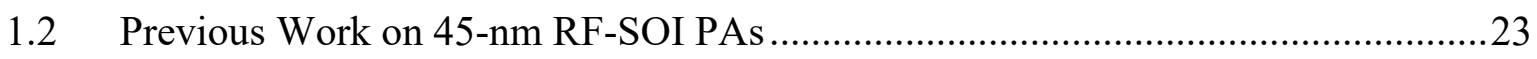

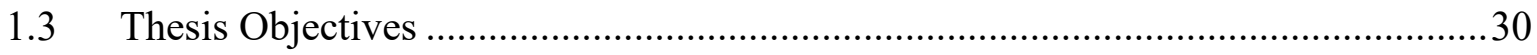

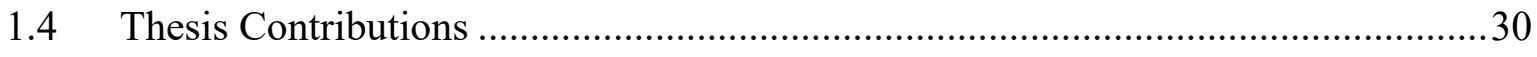

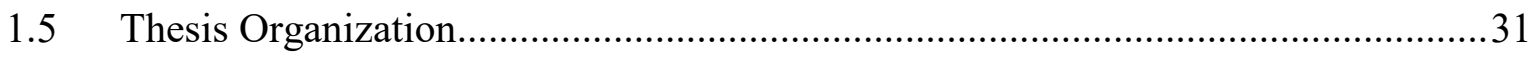

Chapter 2: Principles of Power Amplifiers ...................................................................................32

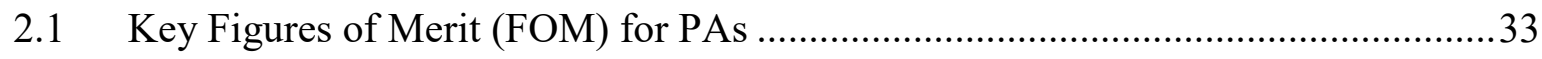

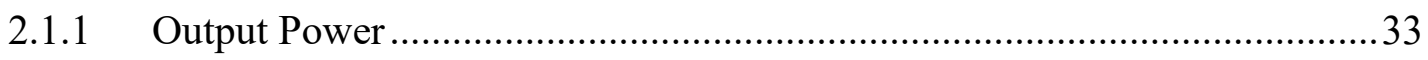

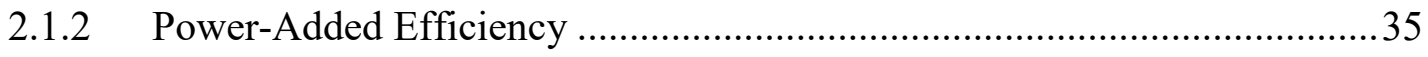

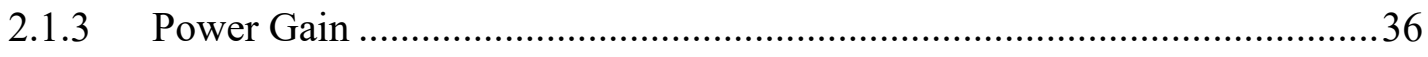

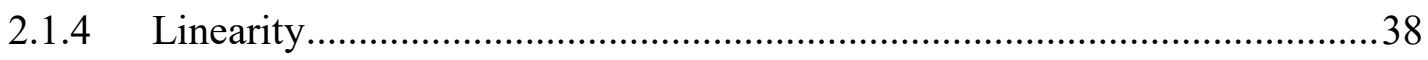

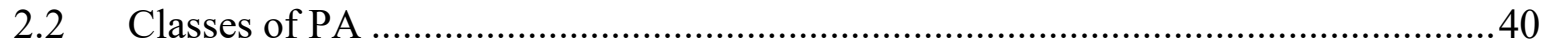

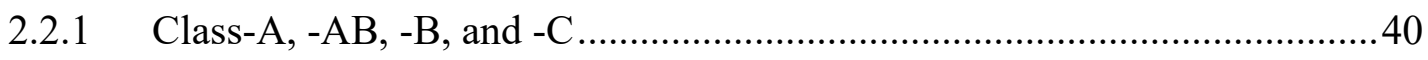

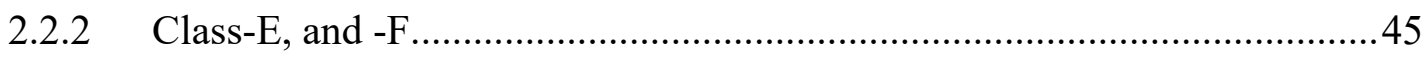

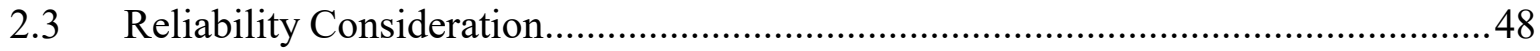

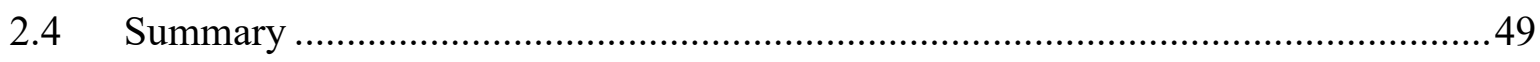

Chapter 3: RF-SOI Technology .....................................................................................................50

$3.1 \quad$ RF-SOI Process Overview …………………………………………………...51

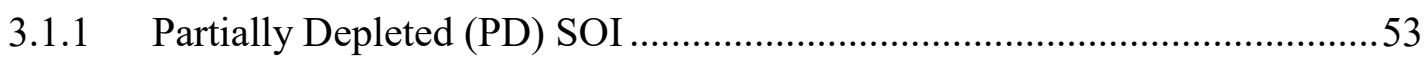

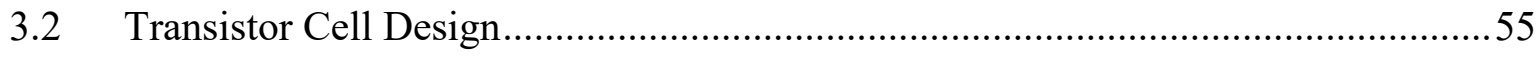

3.2.1 Key FOM for Active Device (relevant to PA Design) .................................56 


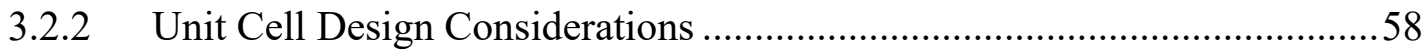

3.2.3 Unit Cell Layout Optimization ...................................................................6

3.2.4 Power Transistor Cell Layout ......................................................................65

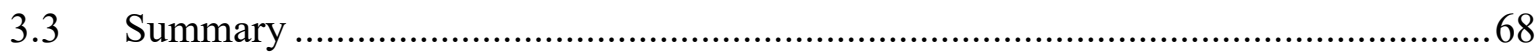

Chapter 4: 28 GHz 3-Stack Power Amplifier in 45-nm RF-SOI...........................................70

$4.1 \quad$ Transistor Stacking Overview ..........................................................................

4.1.1 Determination of Gate Capacitance ............................................................73

4.1.2 Intermediate Node Matching .................................................................

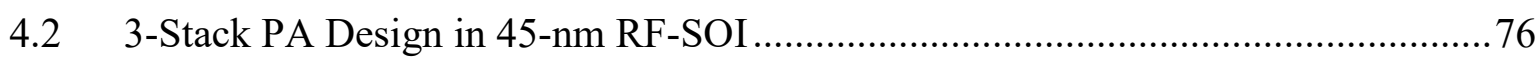

4.2.1 Layout-driven Design and Implementation .................................................77

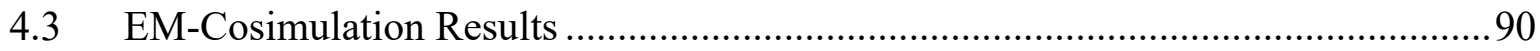

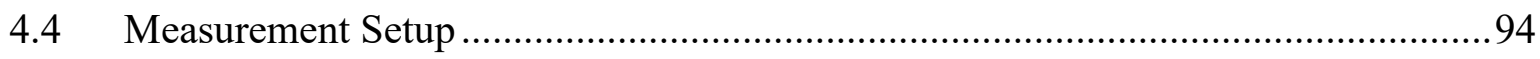

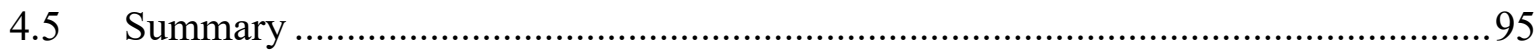

Chapter 5: Conclusions and Future Work .........................................................................96

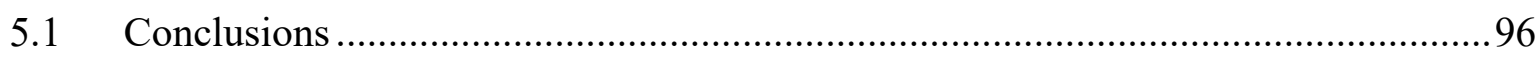

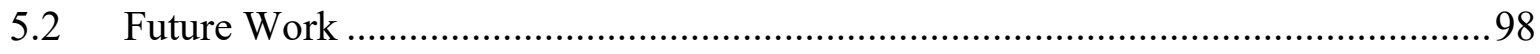

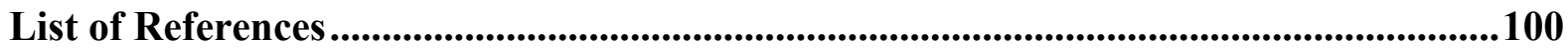




\section{List of Tables}

Table 1.1: Required RF output power from base station and per PA element for different cell

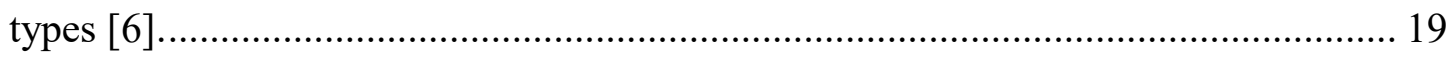

Table 1.2: Previously reported Ka-band $N$-stack PAs in 45-nm RF-SOI. .................................... 29

Table 2.1: Performance comparison of the different classes of conventional PAs. ...................... 44

Table 3.1: Computed small-signal equivalent circuit parameters (at $28 \mathrm{GHz}$ ) of the

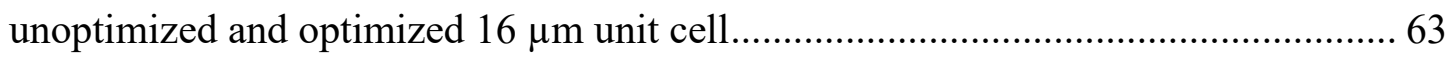

Table 3.2: Computed small-signal equivalent circuit parameters (at $28 \mathrm{GHz}$ ) of the $256 \mu \mathrm{m}$

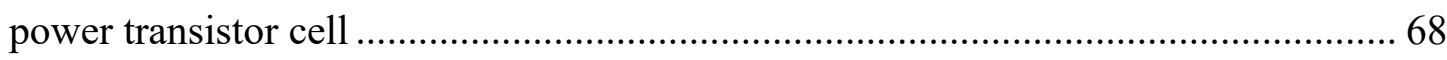

Table 4.1: Computed and optimized values for the external gate capacitor.................................. 79

Table 4.2: Inductance, quality factor, and self-resonant frequency of the input and output

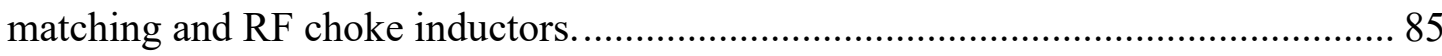

Table 4.3: Performance summary of the 3-stack PA. ………………………………………........ 94 


\section{List of Figures}

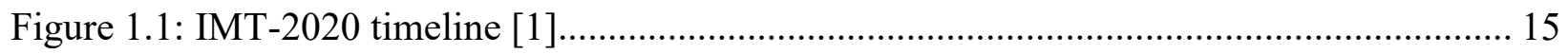

Figure 1.2: Sub-6 GHz and mmWave candidate spectrums for 5G [2] ................................. 17

Figure 1.3: Hybrid beamforming transceiver for 5G mmWave wireless communication [9]...... 20

Figure 1.4: Integration of RF-FEM and an antenna substrate [10]........................................ 17

Figure 1.5: $f_{\max }$ of bulk CMOS versus technology nodes, and $f_{\max }$ of 45-nm RF-SOI [8]............ 22

Figure 1.6: 2-stack PA, which exploits the coupled-inductor neutralization technique to

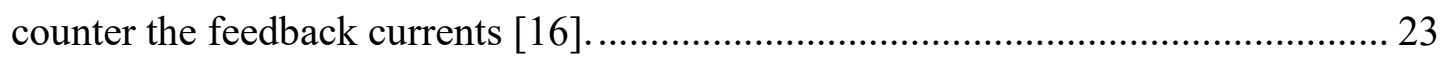

Figure 1.7: 3-stack PA, which exploits transistor sizing to eliminate the output matching

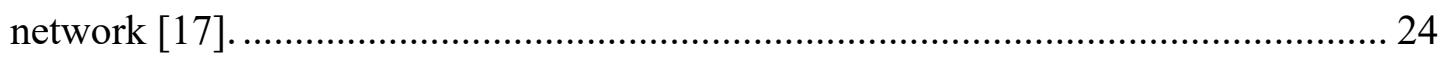

Figure 1.8: 2-stack PA implemented with PMOS devices [18] ................................................ 25

Figure 1.9: Doherty PA, which employs 2-stack PAs for the main and auxiliary amplifiers

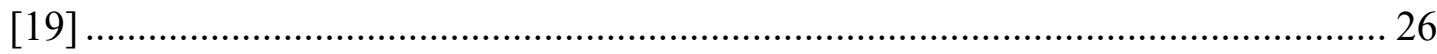

Figure 1.10: 4-stack transistors realized using multigate-cell layout [21] ................................... 27

Figure 1.11: 4-stack PA, which employs multi-gate cell layout [21]........................................ 28

Figure 2.1: Optimum load line and quiescent point that results in maximum output voltage and current excursions (for ideal class-A operation). ............................................... 34

Figure 2.2: Output power, drain efficiency, and power-added efficiency versus input power..... 35

Figure 2.3: Basic concepts of the small-signal conjugate match (top) and large-signal power match (bottom).

Figure 2.4: Output power of a PA under two different matching conditions, small-signal conjugate match (red) and large-signal power match (blue)...................................... 37

Figure 2.5: Gain compression and phase shift in the output signal. ............................................. 39

Figure 2.6: Load lines for different classes of operation and corresponding output voltage

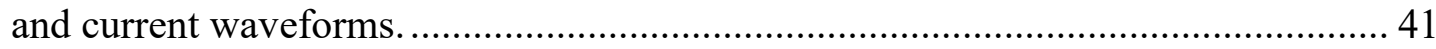

Figure 2.7: Normalized maximum output power versus conduction angle .................................. 43

Figure 2.8: Fourier analysis of reduced conduction angle current waveforms.............................. 44

Figure 2.9: Basic configuration of class-E PA and corresponding drain voltage and current waveforms. 
Figure 2.10: Basic configuration of class-F PA and corresponding drain voltage and current waveforms.

Figure 3.1: RF/mmWave-optimized BEOL metal stack.................................................. 52

Figure 3.2: Cross-section of a floating-body PD-SOI device. ............................................... 54

Figure 3.3: Peak intrinsic $f_{\max }$ versus number of gate fingers for $16 \mu \mathrm{m}$ unit cell ..................... 59

Figure 3.4: Unoptimized (top) and optimized (bottom) $16 \mu \mathrm{m}$ unit cell layout. ........................ 59

Figure 3.5: Staircase routing to minimize source-drain capacitive coupling [37].................... 62

Figure 3.6: High-frequency small-signal equivalent circuit of a transistor ............................. 63

Figure 3.7: Extrinsic $f_{\max }$ versus drain current density for optimized and unoptimized $16 \mu \mathrm{m}$ unit cell.

Figure 3.8: Different styles of power transistor cell layout; in-line (left), round-table (middle), zipper (right) $[11,38,39]$. 65

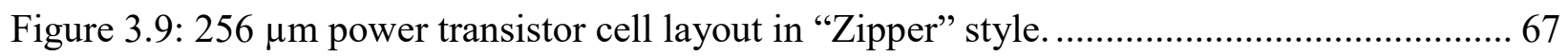

Figure 3.10: Extrinsic $f_{\max }$ versus drain current density for $256 \mu \mathrm{m}$ power transistor cell. ......... 68

Figure 4.1: Basic configuration of an $N$-stack PA. 71

Figure 4.2: Achievable increase in the saturated output power from a common-source PA versus number of stacked devices [40]. 73

Figure 4.3: Intermediate note matching techniques: (a) shunt capacitor (b) series inductor (c) shunt inductor. 73

Figure 4.4: EM-cosimulation test bench of the 3-stack PA. 75

Figure 4.5: Floor plan of the PA chip with bias lines and no input and output matching networks 76

Figure 4.6: Global ground plane on LD layer 77

Figure 4.7: Placement of edge ports to capture the effects of the interconnects and via inductances, as well as the coupling between the interconnects (OB down to UA)

Figure 4.8: Realization of the external gate capacitor per stack using two identical capacitors of value $0.5 C_{k}$. 71

Figure 4.9: VNCAP made with four consecutive metal layers (M1 to C1).... 80

Figure 4.10: On-chip double-layer spiral inductor made with spirals on OA and OB layer 84 
Figure 4.11: Impedance looking towards the RF input pad $\left(Z_{\text {in_RFin_PAD }}\right)$ and impedance looking into the input of the PA $\left(Z_{\text {in_Gate }}\right)$.

Figure 4.12: Load tuner connection point and the impedance looking towards the RF output $\operatorname{pad}\left(Z_{\text {in_RFout } P A D}\right)$.

Figure 4.13: Sampled load points and PAE contours in $0.5 \%$ steps for $19.5 \mathrm{dBm}$ output power.

Figure 4.14: Impedance transformation from $Z_{\text {in_RFout } P A D}$ to $Z_{\text {opt }}$ at $28 \mathrm{GHz}$ (loaded $Q=$ $0.68)$. 88

Figure 4.15: Designed single-ended 3-stack PA, $V_{D D}=3.3 \mathrm{~V}, V_{G 1}=0.55 \mathrm{~V}, V_{G 2}=1.65 \mathrm{~V}, V_{G 3}$ $=2.75 \mathrm{~V}$. 89

Figure 4.16: Layout implementation of the single-ended 3-stack PA $(950 \mu \mathrm{m} \times 700 \mu \mathrm{m})$........ 89

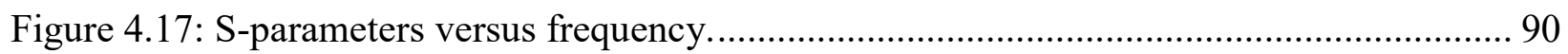

Figure 4.18: Gain and power-added efficiency versus output power at $28 \mathrm{GHz} . \ldots \ldots \ldots \ldots \ldots \ldots \ldots . . . . . . . . . .91$

Figure 4.19: Power-added efficiency and saturated output power across $20 \%$ fractional

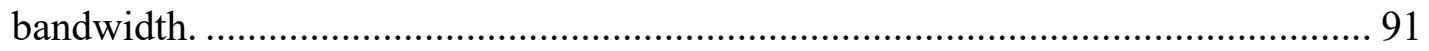

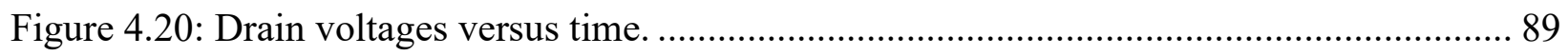

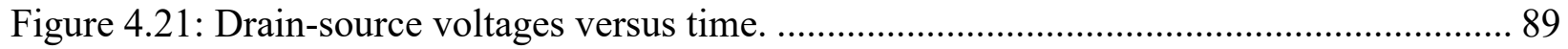

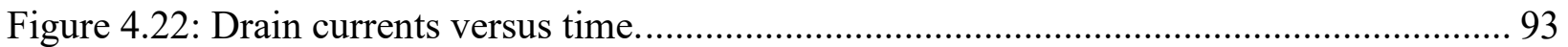

Figure 4.23: AM-AM and AM-PM conversion at $28 \mathrm{GHz}$.............................................. 93

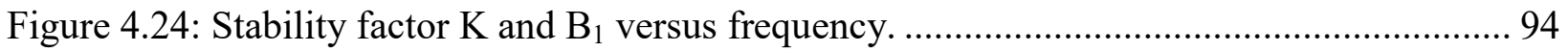

Figure 4.25: Measurement setup for the fabricated chip [40] ........................................... 95 


\section{List of Acronyms and Symbols}

\section{Acronyms}

\begin{tabular}{|c|c|}
\hline Acronym & Definition \\
\hline $5 \mathrm{G}$ & Fifth Generation Wireless System \\
\hline $\mathrm{AC}$ & Alternating Current \\
\hline ACPR & Adjacent Channel Power Ratio \\
\hline BEOL & Back End of Line \\
\hline $\mathrm{BOX}$ & Buried Oxide \\
\hline BVDSS & Drain-source Breakdown Voltage \\
\hline $\mathrm{BW}$ & Bandwidth \\
\hline $\mathrm{CG}$ & Common-Gate \\
\hline CMOS & Complementary Metal Oxide Semiconductor \\
\hline CS & Common-Source \\
\hline $\mathrm{CW}$ & Continuous Wave \\
\hline $\mathrm{DC}$ & Direct Current \\
\hline DPD & Digital Pre-Distortion \\
\hline DSP & Digital Signal Processing \\
\hline DUT & Device Under Test \\
\hline EM & Electromagnetic \\
\hline eMBB & Enhanced Mobile Broadband \\
\hline EER & Envelope Elimination and Restoration \\
\hline ET & Envelope Tracking \\
\hline
\end{tabular}




\begin{tabular}{|l|l|}
\hline EVM & Error Vector Magnitude \\
FET & Field Effect Transistor \\
GaAs & Figure of Merit \\
GaN & Gallium Arsenide \\
HBT & Gallium Nitride \\
HCI & Heterojunction Bipolar Transistor \\
HEMT & Hot Carrier Injection \\
HR & High-Electron-Mobility Transistor \\
IC & High-Resistivity \\
ILD & Integrated Circuit \\
InP & Inter Level Dielectric \\
IP3 & Indium Phosphide \\
LDD & Third-Order Intercept Point \\
LNA & Lightly Doped Drain \\
LOS & Low Noise Amplifier \\
MAG & Line-of-Sight \\
MIM & Maximum Available Gain \\
MIMO & Metal-Insulator-Metal \\
MOF & Multiple Input and Multiple Output \\
\hline
\end{tabular}




\begin{tabular}{|l|l|}
\hline PA & Power Amplifier \\
PAE & Power-Added Efficiency \\
PEX & Peak-to-Average Power Ratio \\
PD & Parasitic Extraction \\
QAM & Partially Depleted \\
RF & Quadrature Amplitude Modulation \\
RF-FEM & Radio Frequency \\
SCE & RF Front-End Modules \\
SiGe & Short-Channel Effects \\
SiO & Silicon Germanium \\
SNR & Silicon Dioxide \\
SOI & Signal-to-Noise Ratio \\
SOLT & Silicon-On-Insulator \\
SRF & Short-Open-Load-Thru \\
STI & Self-Resonant Frequency \\
TDDB & Shallow Trench Isolation \\
TRL & Time-Dependent Dielectric Breakdown \\
UGW & Thru-Reflect-Load \\
uRLLC & Unit Gate Width \\
VGA & Ultra-Reliable and Low Latency Communication \\
\hline
\end{tabular}




\section{Symbols}

\begin{tabular}{|c|c|}
\hline Acronym & Definition \\
\hline$\eta_{\text {drain }}$ & Drain Efficiency [\%] \\
\hline$\Gamma_{\text {opt }}$ & Optimum Load Reflection Coefficient \\
\hline$\lambda$ & Wavelength $[\mathrm{m}]$ \\
\hline$\theta$ & Phase $\left[{ }^{\circ}\right]$ \\
\hline$C_{d s}$ & Drain-Source Capacitance [F] \\
\hline$C_{g d}$ & Gate-Drain Capacitance $[\mathrm{F}]$ \\
\hline$C_{g s}$ & Gate-Source Capacitance [F] \\
\hline$f_{\max }$ & Maximum Oscillation Frequency $[\mathrm{Hz}]$ \\
\hline$f_{o}$ & Operating Frequency [Hz] \\
\hline$f_{T}$ & Transit Frequency $[\mathrm{Hz}]$ \\
\hline$G$ & Power Gain $[\mathrm{dB}]$ \\
\hline$g_{d s}$ & Output Conductance $\left[\Omega^{-1}\right]$ \\
\hline$g_{m}$ & Transconductance $[\mathrm{S}]$ \\
\hline$I_{\max } / I_{\min }$ & Maximum/Minimum Output Current [A] \\
\hline$P_{1 d B}$ & 1-dB Compression Point [W] \\
\hline$P_{D C}$ & DC Power [W] \\
\hline$P_{R F_{-} \text {out }}$ & RF Output Power [W] \\
\hline$P_{\text {sat }}$ & Saturated Output Power [W] \\
\hline$Q$ & Quality Factor \\
\hline$r_{d s}$ & Drain-Source Resistance [ $\Omega]$ \\
\hline$R_{L}$ & Load Resistance $[\Omega]$ \\
\hline
\end{tabular}




\begin{tabular}{|l|l|}
\hline$R_{o p t}$ & $\begin{array}{l}\text { Optimum Load Resistance }[\Omega] \\
V_{B S}\end{array}$ \\
$V_{D D}$ & Body-Source Voltage [V] \\
$V_{k n e e}$ & KC Power Supply Voltage [V] \\
$V_{t}$ & Threshold Voltage [V] \\
$Z_{\text {opt }}$ & Optimum Load Impedance $[\Omega]$ \\
\hline
\end{tabular}




\section{Chapter 1}

\section{Introduction}

With the standardization process for International Mobile Telecommunications-2020 (IMT-2020) rapidly approaching its completion [1], the fifth-generation wireless communication $(5 \mathrm{G})$ is slowly but surely becoming a reality. It is forecasted that $5 \mathrm{G}$ ecosystem will be comprised of three main use cases, namely, enhanced mobile broadband (eMBB); ultra-reliable, and low latency communication (uRLLC); and massive machine-type communications (mMTC); where these use cases are expected to drive $5 \mathrm{G}$ extremely data-centric by nature [2]. Hence, in order to cope with the explosive growth of data traffic, $5 \mathrm{G}$ aims to achieve a 1000 -fold increase in system capacity (per $\mathrm{km}^{2}$ ), greater than $10 \mathrm{Gbps}$ peak data rates (downlink), and sub-1ms latency $[2,3]$.

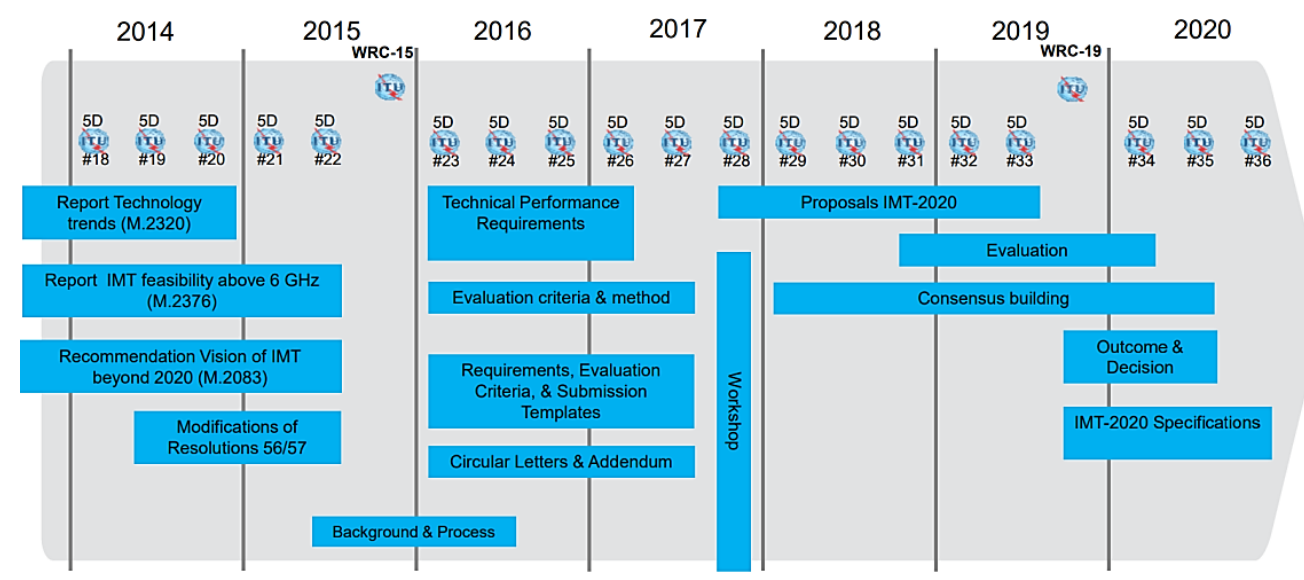

Figure 1.1: IMT-2020 timeline [1]. 
Along with the use of spectrally efficient high-order modulation schemes such as 256 or 1024 quadrature amplitude modulation (QAM), the key technologies that will enable 5G have been identified to be the adoption of millimeter-wave (mmWave) spectrum; small cell densification; massive multiple-input and multiple-output (MIMO); and electronic beam forming and steering using phased-array antennas [2]. Each of these technologies contributes to enhancing the individual parameters in the well-known Shannon-Hartley's channel capacity theorem, thereby enabling higher channel capacity. Shannon-Hartley's channel capacity theorem can be expressed as shown in (1.1) where $C$ is the channel capacity in bps, $M$ is the MIMO order, $B W$ is the bandwidth in $\mathrm{Hz}$, and $S N R$ is the signal-to-noise ratio.

$$
C=M \cdot B W \cdot \log _{2}(1+S N R)
$$

Among the aforementioned key technologies for $5 \mathrm{G}$, the adoption of mmWave spectrum for commercial $5 \mathrm{G}$ services is considered particularly remarkable and groundbreaking. It is anticipated that the large bandwidths available in the mmWave spectrum will alleviate the spectrum scarcity problem currently existing in the sub-6 GHz spectrum while playing a key role in achieving the target peak data rates [3].

While mmWave wireless communication sounds fascinating and promising, it has several known drawbacks, such as higher path losses due to oxygen absorption and lower received SNR due to small antenna aperture [11]. Consequently, these drawbacks necessitate short-range (100$200 \mathrm{~m}$ ) and point-to-point line-of-sight (LOS) communication at mmWave frequencies. To this end, small cell densification and steerable phased array antennas have been proposed as viable solutions $[4,5]$. Another drawback of mmWave wireless communication is that the battery life of battery-powered devices is expected to be reduced considerably as more current will be drawn 
from the battery. Consequently, the use of low power consumption ICs has become increasingly important.

\subsection{Motivation for 28 GHz RF-SOI PA}

It has been announced that the initial deployment of $5 \mathrm{G}$ network will be in the sub- $6 \mathrm{GHz}$ bands (phase 1), followed by mmWave bands (phase 2) [1-3]. Figure 1.2 shows the sub-6 GHz, and mmWave candidate spectrums for $5 \mathrm{G}$, and their associated bandwidths for different countries. It can be seen that in the mmWave spectrum, Ka-band $(26.5-40 \mathrm{GHz})$, and particularly $28 \mathrm{GHz}$, has drawn keen attention from all countries (except China); the reason for such high interest in $28 \mathrm{GHz}$ is attributed to the fact that the propagation losses at $28 \mathrm{GHz}$ are moderate compared to other higher mmWave bands $[4,5]$. Consequently, it is anticipated that there will be an exponential increase in the number of handsets and small-cell radios that can support $28 \mathrm{GHz}$ band, which, in turn, translates to an increase in the demand for cost-effective mmWave power amplifiers (PAs).

Sub-6 GHz Spectrum (MHz) Millimeter Wave Spectrum (GHz)

\begin{tabular}{|c|c|c|c|c|c|c|c|c|c|c|c|}
\hline \multirow[b]{2}{*}{ Region } & \multicolumn{2}{|c|}{ New } & \multicolumn{3}{|c|}{ Existing } & \multirow{2}{*}{\multicolumn{2}{|c|}{ Total }} & \multirow[b]{2}{*}{$F_{\text {HIGH }}$} & \multirow[b]{2}{*}{ Band } & \\
\hline & $\mathbf{F}_{\text {Low }}$ & $F_{\text {HIGH }}$ & $F_{\text {Low }}$ & $\mathrm{F}_{\text {HIGH }}$ & Band & & BW & & & BW & BW \\
\hline Korea & 3400 & 3700 & & & & 300 & 300 & 26.50 & 29.50 & 3.00 & 3.00 \\
\hline EU & 3400 & 3800 & $\begin{array}{l}2570 \\
3400\end{array}$ & $\begin{array}{l}2620 \\
3800\end{array}$ & $\begin{array}{c}38 \\
42+43\end{array}$ & $\begin{array}{c}50 \\
400\end{array}$ & \multirow{4}{*}{1494} & \multirow{2}{*}{$\begin{array}{l}24.25 \\
31.80 \\
40.50\end{array}$} & \multirow{2}{*}{$\begin{array}{l}27.35 \\
33.40 \\
43.50\end{array}$} & \multirow{2}{*}{$\begin{array}{l}3.10 \\
1.60 \\
3.00 \\
\end{array}$} & \multirow[t]{2}{*}{7.70} \\
\hline \multirow{3}{*}{ Japan } & \multirow{3}{*}{$\begin{array}{l}3600 \\
4400\end{array}$} & \multirow{3}{*}{$\begin{array}{l}4200 \\
4900\end{array}$} & \multirow{3}{*}{$\begin{array}{l}2496 \\
3400\end{array}$} & \multirow{3}{*}{$\begin{array}{l}2690 \\
3600\end{array}$} & \multirow{3}{*}{$\begin{array}{l}41 \\
42\end{array}$} & \multirow{3}{*}{$\begin{array}{l}194 \\
800 \\
500\end{array}$} & & & & & \\
\hline & & & & & & & & 27.50 & 29.50 & 2.00 & 2.00 \\
\hline & & & & & & & & 27.50 & 28.35 & 0.85 & \multirow{4}{*}{10.85} \\
\hline \multirow{2}{*}{ U.S. } & & & 2496 & 2690 & 41 & 194 & \multirow{2}{*}{344} & \multirow{3}{*}{$\begin{array}{l}37.00 \\
38.60 \\
64.00\end{array}$} & \multirow{3}{*}{$\begin{array}{l}38.60 \\
40.00 \\
71.00\end{array}$} & \multirow{3}{*}{$\begin{array}{l}1.60 \\
1.40 \\
7.00\end{array}$} & \\
\hline & & & 3550 & 3700 & 48 & 150 & & & & & \\
\hline \multirow{4}{*}{ China } & & & 2300 & 2400 & 40 & 100 & & & & & \\
\hline & & & 2555 & 2655 & 41B & 100 & & & & & \\
\hline & 3300 & 3600 & 3400 & 3600 & 42 & 300 & 790 & \multirow{2}{*}{\multicolumn{4}{|c|}{$\begin{array}{l}\text { Bold values indicate newly introduc } \\
\text { frequency ranges with worldwide }\end{array}$}} \\
\hline & $\begin{array}{l}4400 \\
4800\end{array}$ & $\begin{array}{l}4500 \\
4990\end{array}$ & & & & $\begin{array}{l}100 \\
190\end{array}$ & & & & & \\
\hline
\end{tabular}

Figure 1.2: Sub-6 GHz and mmWave candidate spectrums for 5G [2]. 
Traditionally, III-V processes such as Gallium Arsenide (GaAs), Indium Phosphide (InP), and Gallium Nitride $(\mathrm{GaN})$ have been favored over complementary metal-oxide semiconductor (CMOS) for implementing mmWave PAs; this is because III-V processes offer devices with higher voltage handling capability and the high resistivity III-V substrates allow the realization of low-loss transmission lines and high-quality factor $(Q)$ on-chip passives $[6,7]$. These features have allowed the III-V PAs to achieve RF performance (i.e., output power, efficiency, linearity, and bandwidth) superior to that of CMOS counterparts. Nevertheless, while RF performance is a critical factor when it comes to deciding on a process for implementing mmWave PAs, the capability of a process to offer a high level of integration, low power consumption, and economies of scale become equally as critical concerning 5G mmWave PAs.

CMOS has been the process of choice for digital, analog, and mixed-signal circuits for many years. However, despite the high transit frequency $f_{T}$ and maximum oscillation frequency $f_{\max }\left(f_{\max }>250 \mathrm{GHz}[8]\right)$ attained from continuous technology scaling (which has been driven by the advancement in the high-performance digital ICs), CMOS has not been the primary choice of process for implementing mmWave PAs; this is because CMOS devices have inherently low voltage handling capability, and hence extracting RF output power comparable to that of III-V is rather difficult or requires the use of very sophisticated circuit techniques and/or topologies. Furthermore, the lossy transmission lines and on-chip passives (primarily the integrated inductors) resulting from the low resistivity silicon substrate $(\sim 10 \Omega-\mathrm{cm}[11])$ degrades the RF performance of the PA [7]. It is indisputable that III-V processes have clear advantages over CMOS in terms of having the capability to achieve the desired RF performance more readily; however, for reasons that will become clear shortly, CMOS turns out to be a more attractive process for mmWave PAs that are to be used in 5G handsets and small-cell radios. 
Studies show that with the small cell densification and the use of phased-array antennas, the RF output power requirement per PA element from a small-cell radio or a handset is expected to drop to 50-300 $\mathrm{mW}$ (or 17-24 $\mathrm{dBm}$ ) range [6]. It can be seen from Table 1.1 that the $\mathrm{RF}$ output power per PA element for femto and picocells (which are the two cell types that correspond to a small cell) is less than $20 \mathrm{dBm}$ (or $100 \mathrm{~mW}$ ). The drop in the RF output power level works in favor of CMOS, since power is at a premium for CMOS.

Table 1.1: Required RF output power from base station and per PA element for different cell types [6].

\begin{tabular}{|c|c|c|}
\hline $\begin{array}{c}\text { Cell } \\
\text { Type }\end{array}$ & $\begin{array}{c}\text { RF power from base station } \\
{[\mathbf{d B m}]}\end{array}$ & $\begin{array}{c}\text { RF output power per PA element } \\
\text { [dBm] }\end{array}$ \\
\hline Femto & $<24$ & $<20$ \\
\hline Pico & $24-30$ & $<20$ \\
\hline Micro & $30-40$ & $<27$ \\
\hline Macro & $40-47$ & $>27$ \\
\hline
\end{tabular}

The RF-FEMs in the traditional mmWave transceivers have a low level of integration as each active component in the RF-FEM (i.e., PA, LNA, switch, phase shifter, and VGA) is implemented in a process that results in the best performance for that specific component (e.g., GaAs or InP HBT/HEMT, SiGe HBT, CMOS, and others) [9]. Consequently, this results in RFFEMs with a large form factor and high costs. For 5G mmWave transceivers, which will most likely adopt the hybrid beamforming architecture shown in Figure 1.3, it is required to minimize the size of RF-FEM so that the massive MIMO system can be realized compactly. To this end, the high integration capability of CMOS can be exploited to integrate all the active components of an RF-FEM into a single die while simultaneously incorporating digital logic and digital signal processing (DSP) features; this allows the realization of a small form factor and low-cost RF-FEM, which, in turn, allows the realization of a compact MIMO system. 


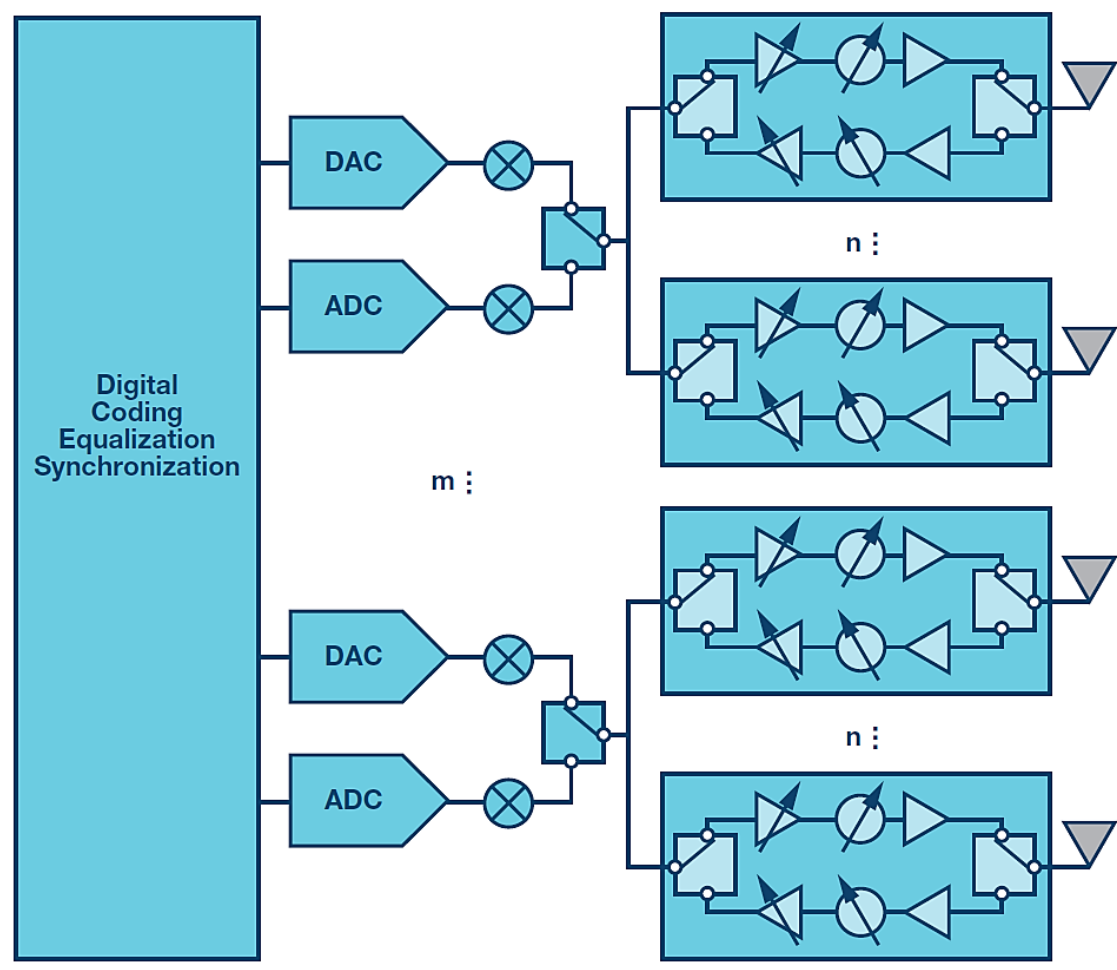

Figure 1.3: Hybrid beamforming transceiver for 5G mmWave wireless communication [9].

In order to take the level of integration even further, it has been proposed to integrate an RF-FEM with an antenna substrate [10] as shown in Figure 1.4. The integration of RF-FEM and antenna substrate becomes feasible at mmWave frequencies, since the physical size of the antenna array and the half-wavelength $(\lambda / 2)$ spacing between the antenna elements becomes small. The wavelength at $28 \mathrm{GHz}$ in free space and $\mathrm{SiO}_{2}$ is $\sim 10.7 \mathrm{~mm}$ and $5.1 \mathrm{~mm}$, respectively. By integrating the RF-FEM with the antenna substrate, the off-chip routing losses, which can be siginificant at mmWave frequencies, can be reduced. Consequently, this leads to improvement in the overall system efficiency.

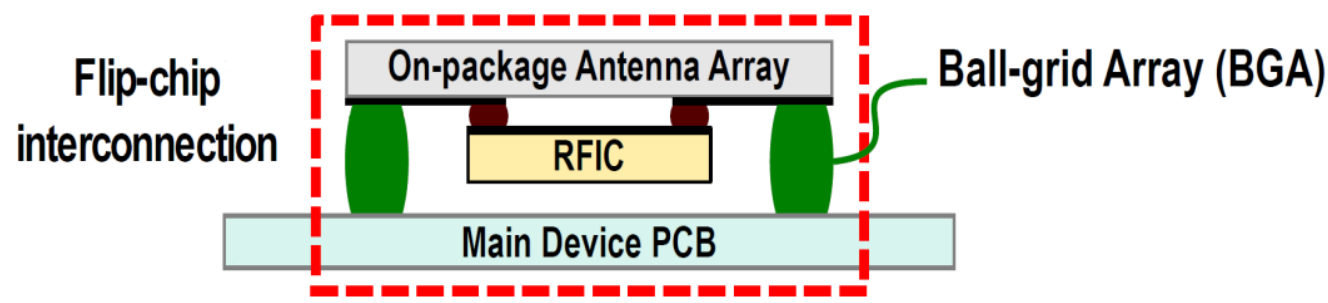

Figure 1.4: Integration of RF-FEM and an antenna substrate [10]. 
When it comes to manufacturing cost, CMOS holds a dominant position over III-V. Being a mature mainstream technology for high-density digital, analog, and mixed signal ICs, it offers excellent yield and clear economies of scale; hence, it is capable of meeting the highvolume demands [11].

Based on what has been described so far, CMOS seems to be capable of meeting the majority of requirements for $5 \mathrm{G}$ mmWave PA. Nevertheless, due to the limited power supply voltage, extracting an adequate amount of RF power from advanced node CMOS devices remains a challenge. In order to overcome this challenge, CMOS PAs typically employ on-chip passive combining (e.g., transformer-based and Wilkinson) to achieve higher RF output power [12]. However, with the on-chip passive combining, compromises on the achievable RF performance have to be made as the losses of the on-chip output combiner often cause degradation of gain and PAE and also limits the bandwidth of the PA. Furthermore, in the case of Wilkinson power combiner, there is an area penalty as the structure consumes a large portion of the die area.

Relatively recently, a technology called RF Silicon-On-Insulator (RF-SOI) has been introduced and is becoming widely adopted in RF/mmWave circuits. RF-SOI retains most of the advantages of the bulk CMOS, yet also offers features that are tailored for RF/mmWave circuits (e.g., high $f_{T} / f_{\text {max }}$ devices and RF/mmWave-optimized substrate and BEOL metal stacks [8, 1315]). Consequently, the problem of lossy transmission lines and on-chip passives that persisted in the bulk CMOS is largely mitigated in RF-SOI. Figure 1.5 shows $f_{\max }$ of bulk CMOS as a function of technology nodes, and $f_{\max }$ of 45 -nm RF-SOI. It can be seen that at the equivalent technology node, $f_{\max }$ of RF-SOI is higher than that of the bulk CMOS as a result of reduced source/drain (S/D) to the substrate parasitic capacitances. It can also be seen that $f_{\max }$ of bulk 
CMOS peaks at 65-nm node and declines for more advanced nodes due to the higher layout parasitics that are added to the intrinsic device core (i.e., higher $R_{g} \times C_{g d}$ product) [8].

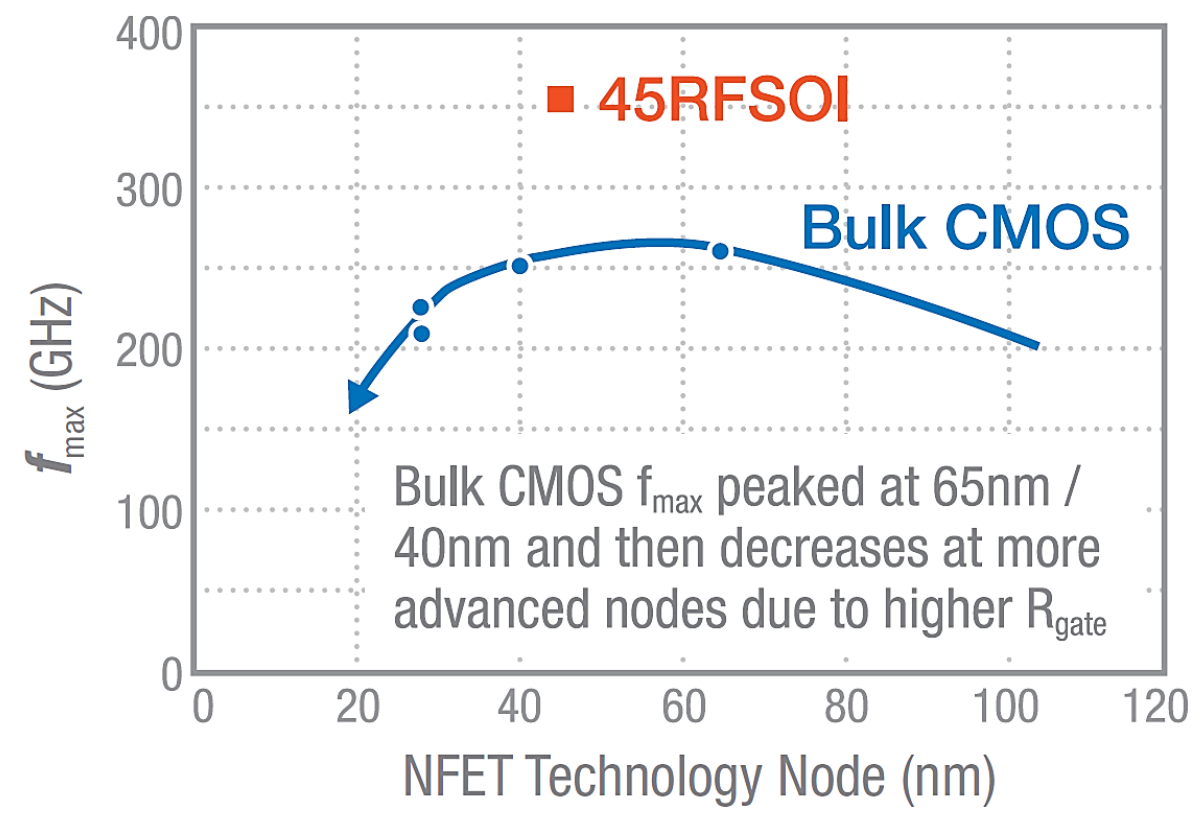

Figure 1.5: $f_{\max }$ of bulk CMOS versus technology nodes, and $f_{\max }$ of 45-nm RF-SOI [8].

In RF-SOI, the presence of a thin buried-oxide (BOX) layer essentially eliminates the body effect and hence facilitates transistor stacking [8, 13-15]. Transistor stacking is another way of boosting the RF output power of a PA, which has not been favored by CMOS PAs, since CMOS devices suffer from the body effect (which is due to the substrate being a common node for all devices). As the body effect degrades the transconductance of the devices considerably and is exacerbated with a higher number of devices in the stack [12], transistor stacking is not an ideal technique to be used with CMOS PAs. On the contrary, since RF-SOI devices do not suffer from the body effect, transistor stacking can be readily used to achieve higher RF output power. Transistor stacking allows higher power supply voltage to be used for the PA as the power supply voltage is distributed equally among the individual transistors in the stack. Furthermore, the voltage swings across the individual transistors in the stack add constructively to result in a 
higher voltage swing at the drain node of the top transistor. Compared to the on-chip passive combining, transistor stacking results in higher PAE and is more area-efficient as the large onchip output combiner can be eliminated. The subject of transistor stacking will be covered in more detail in Chapter 4.

\subsection{Previous Work on 45-nm RF-SOI PAs}

In the last few years, a number of fully integrated Ka-band $N$-stack PAs implemented in 45 -nm RF-SOI have been reported. The reported PAs are targeting the upcoming $5 \mathrm{G} \mathrm{mmWave} \mathrm{wireless}$ communication, where cost-effective PAs are expected to be in high demand.

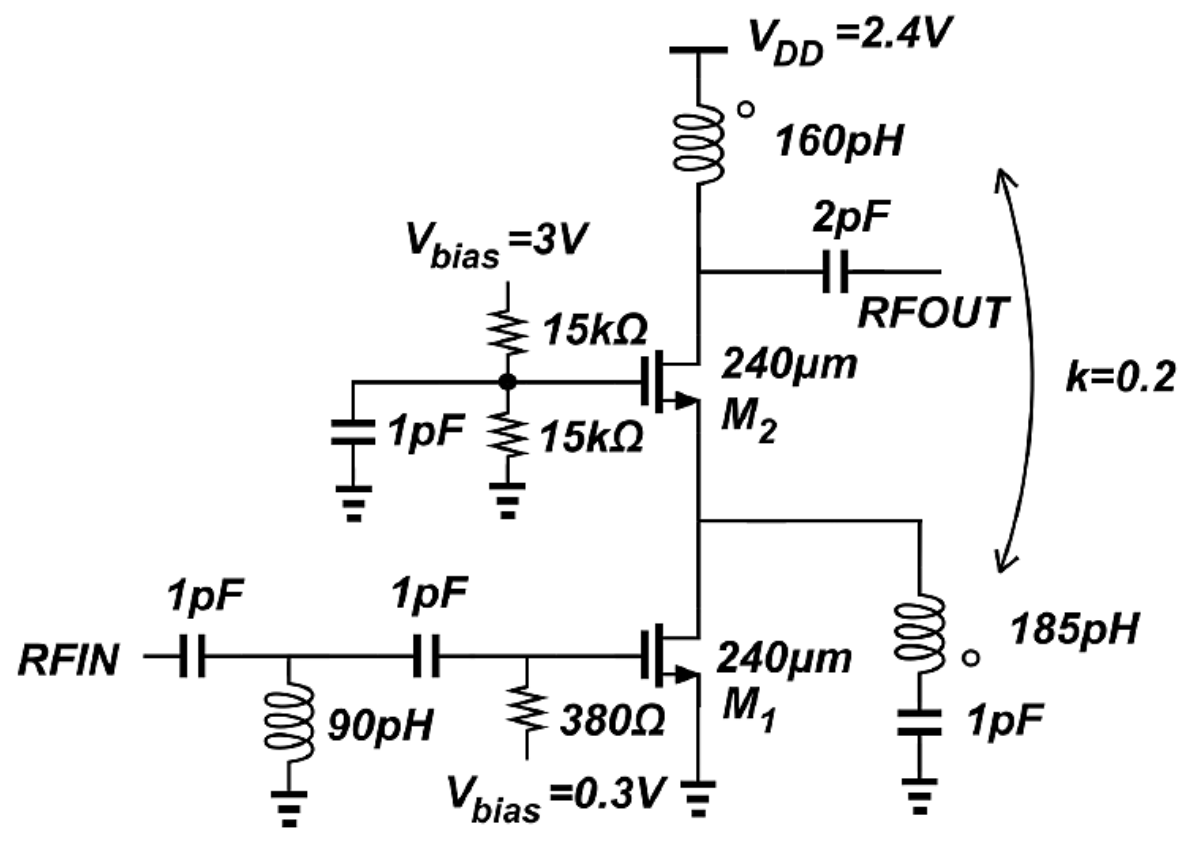

Figure 1.6: 2-stack PA, which exploits the coupled-inductor neutralization technique to counter the feedback currents [16].

A $28 \mathrm{GHz}$, single-stage 2-stack PA, which exploits the coupled-inductor neutralization technique to neutralize the effect of feedback capacitance $C_{g d}$ is reported in [16]. At high frequencies, the feedback current through the parasitic gate-drain capacitance $C_{g d}$ causes the 
drain current $i_{D}$ and the drain-source voltage $v_{D S}$ to be not $180^{\circ}$ out-of-phase; this results in higher power dissipation in the devices, which, in turn, degrades the PAE of a PA. Hence, in order to counter the feedback current, the proposed technique utilizes the shunt inductor, which is originally used for intermediate node matching. First, the shunt inductor is intentionally made to couple to the load inductor. Then, by selecting a proper coupling coefficient, the mutual currents that are $180^{\circ}$ out-of-phase with the feedback currents are induced on the drain nodes of each transistor, which cancels out the feedback currents. The use of this technique resulted in higher PAE for the PA as the power dissipation in the devices is reduced. Furthermore, the use of this technique resulted in higher gain and improved stability of the PA.

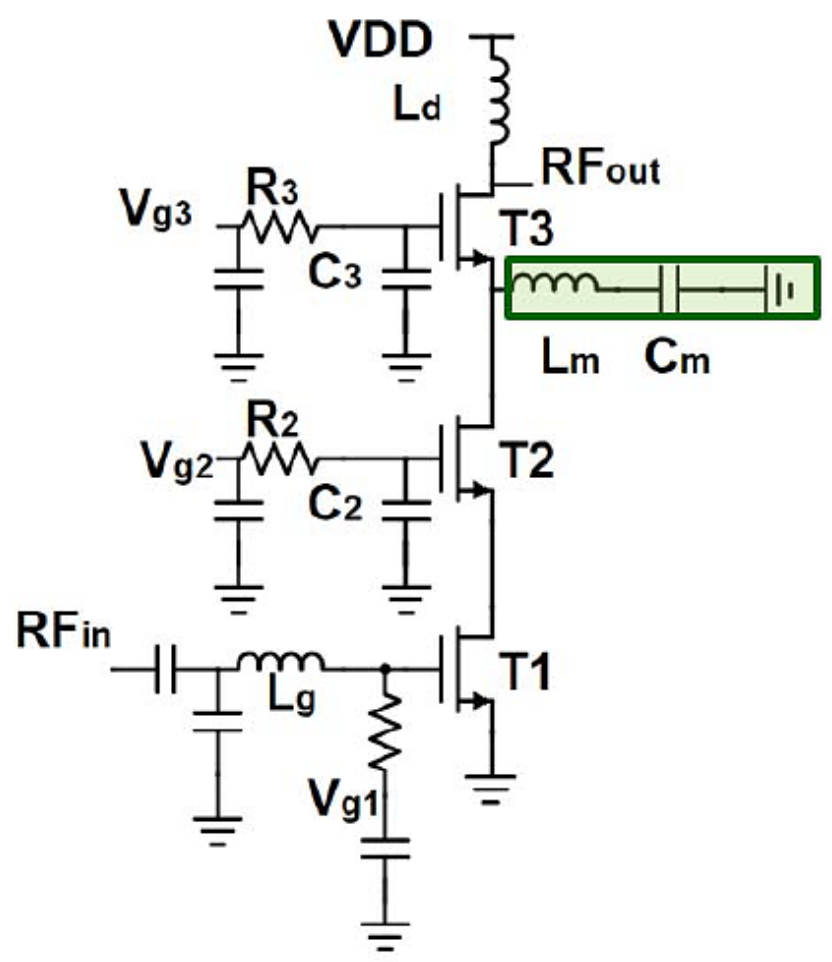

Figure 1.7: 3-stack PA, which exploits transistor sizing and stack height to eliminate the output matching network [17].

A $24 \mathrm{GHz}$, single-stage 3-stack PA, which exploits transistor sizing and stack height to eliminate the output matching network, is reported in [17]. The number of transistors to be 
stacked and the size of the transistors are selected, such that the real part of the optimum load impedance for the PA is close to $50 \Omega$, while the imaginary part is resonated out by the RF choke inductor. Typically, an on-chip output matching network takes a bite out of the output power and degrades the PAE of a PA; hence, eliminating the output matching network and connecting the PA output directly to the load leads to a corresponding improvement in the PA's performance. The reported PA also used intermediate node matching between the top and middle transistors to gain further improvement in PAE.

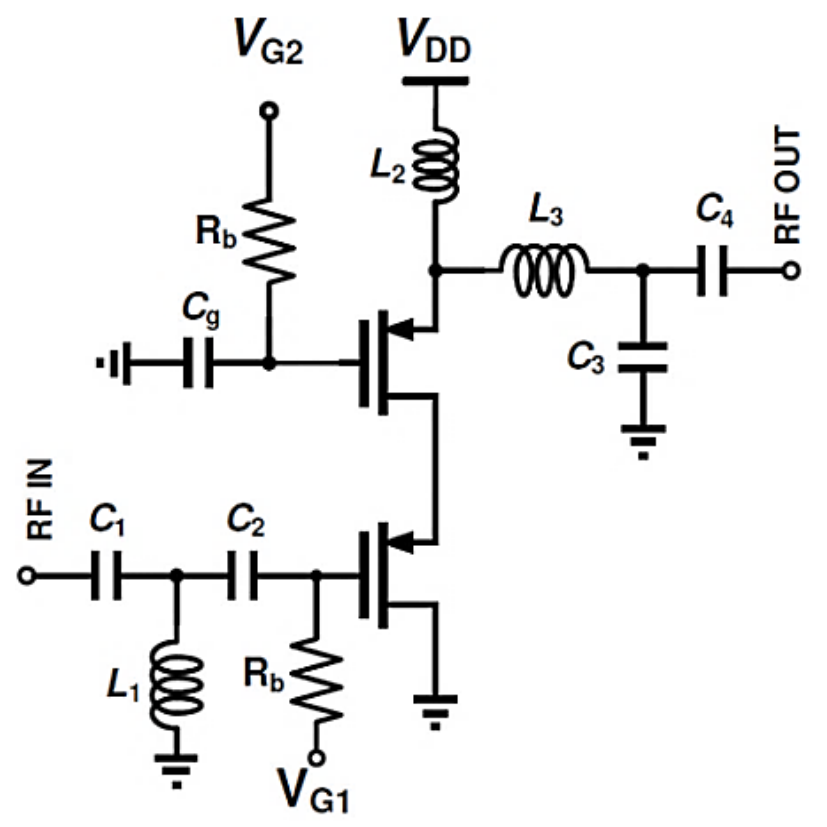

Figure 1.8: 2-stack PA implemented with PMOS devices [18].

A 26.5 GHz, single-stage 2-stack PA implemented using PMOS devices is reported in [18]. Aside from the well-known fact that PMOS devices are slower than the NMOS devices (i.e., lower $\left.f_{T} / f_{\max }\right)$, PMOS devices are less susceptible to hot carrier injection (HCI) and timedependent dielectric breakdown (TDDB). Based on the short-term reliability assessment carried out where the power supply voltage of the PA was varied from $2 \mathrm{~V}$ to $3 \mathrm{~V}$ (in steps $0.2 \mathrm{~V}$ ) while the PA was operated at the saturated output power for 30 minutes at each test voltage, it was 
found that PMOS PAs show less performance degradation compared to its NMOS counterparts. Based on this observation, the author of [18] claims that higher power supply voltage can be used with PMOS PAs to achieve higher output power and efficiency.

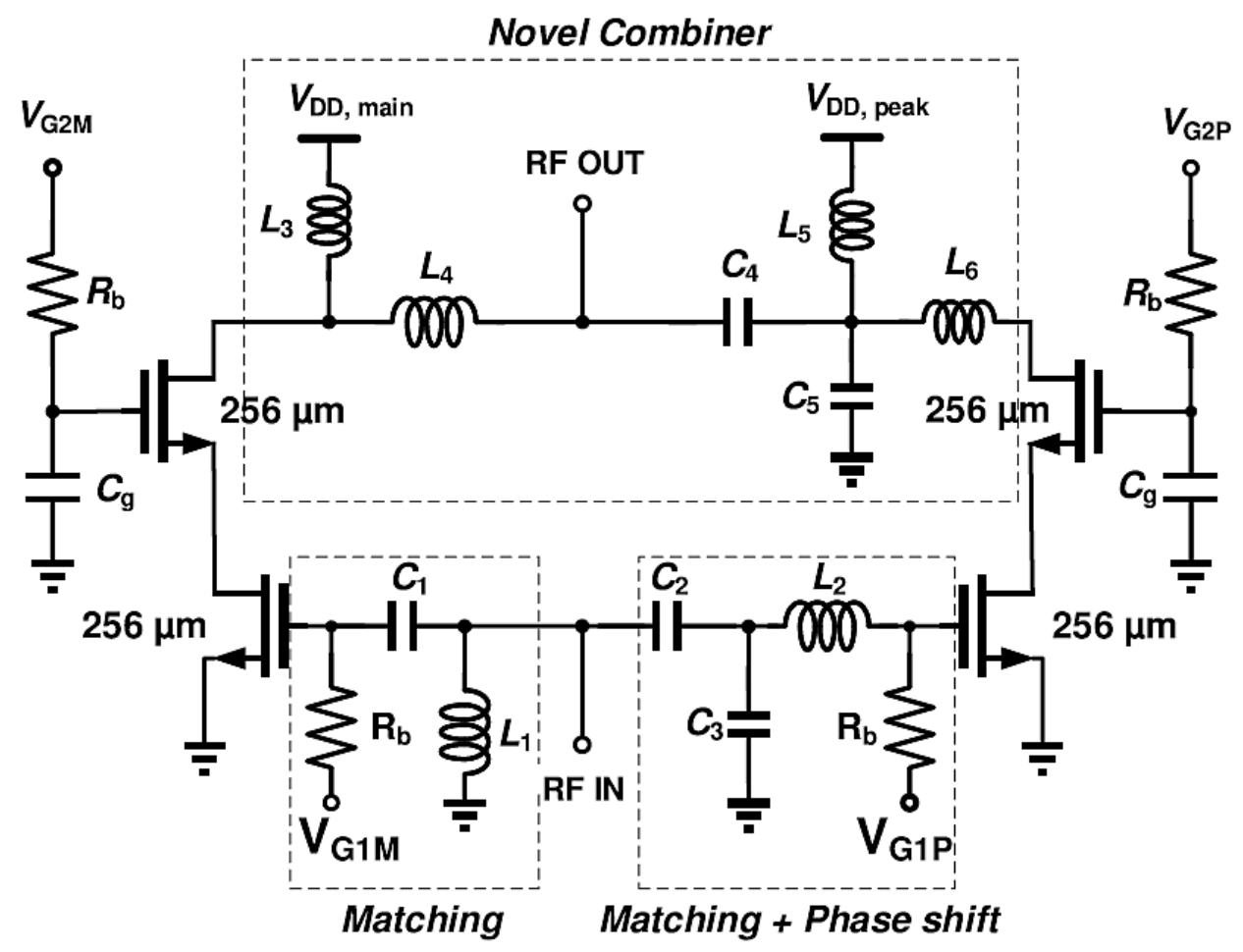

Figure 1.9: Doherty PA, which employs 2-stack PAs for the main and auxiliary amplifiers [19].

A $28 \mathrm{GHz}$, single-stage Doherty PA that employs symmetric 2-stack PAs for the main and auxiliary amplifiers is reported in [19]. Doherty PAs are capable of maintaining high efficiency over a wide signal dynamic range, and hence, they are well-suited for the modern wireless communication system, which uses complex modulated signals with large envelope variations or high peak-to-average power ratio. The Doherty PA reported in [19] uses the lowloss $(0.5 \mathrm{~dB})$ output combiner implemented using the analytical synthesis methodology presented in [20]; the output combiner performs the active load modulation and impedance matching required for the Doherty operation. 

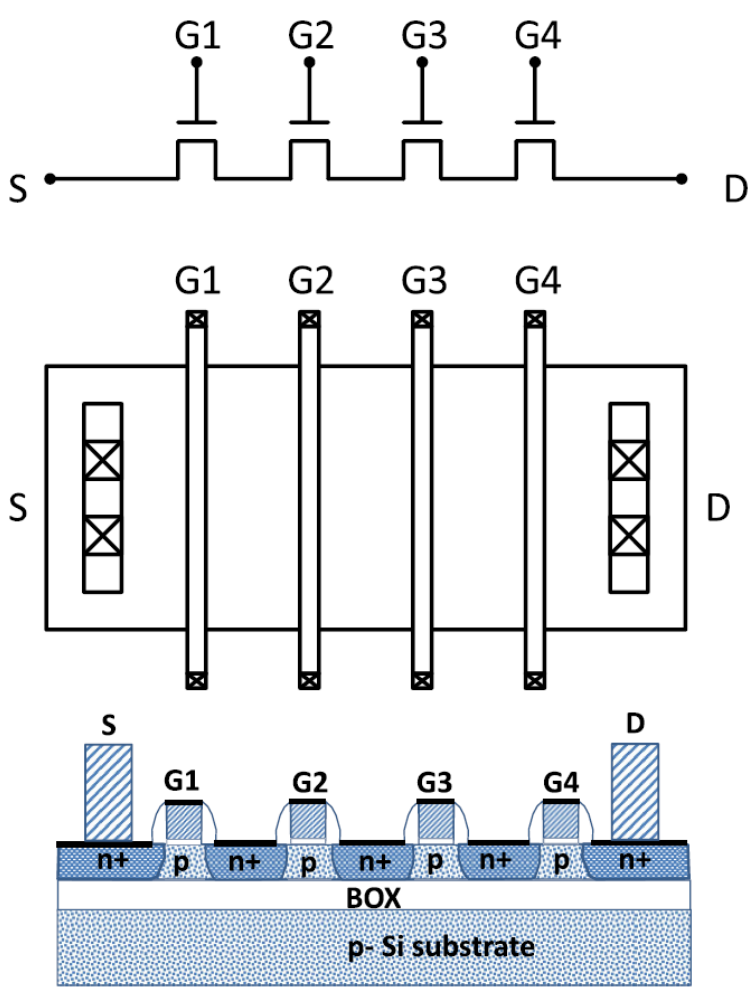

Figure 1.10: 4-stack transistors realized using multigate-cell layout [21].

A $28 \mathrm{GHz}$, single-stage 4-stack PA that employs multigate-cell device layout is reported in [21]. As illustrated in Figure 1.10, the four devices in the stack are implemented as a single device with one source and drain connection, and the four gates sharing a common active area. The advantage of the multigate-cell device layout is that it results in a compact size and has reduced parasitic capacitances and resistances. The large periphery required for the PA is obtained by connecting multiple multigate-cells in parallel, and the external gate capacitors for the upper stack devices are implemented locally within the unit cell. The 4-stack PA implemented using the multigate-cell device layout is broadband due to the absence of tunning elements and is thermally robust. However, a major drawback of employing the multigate-cell layout is that the intermediate node matching cannot be employed to improve the performance of the PA as there is no access to the intermediate nodes. The 4-stack PA, which employs multigate cell layout is shown in Figure 1.11. 


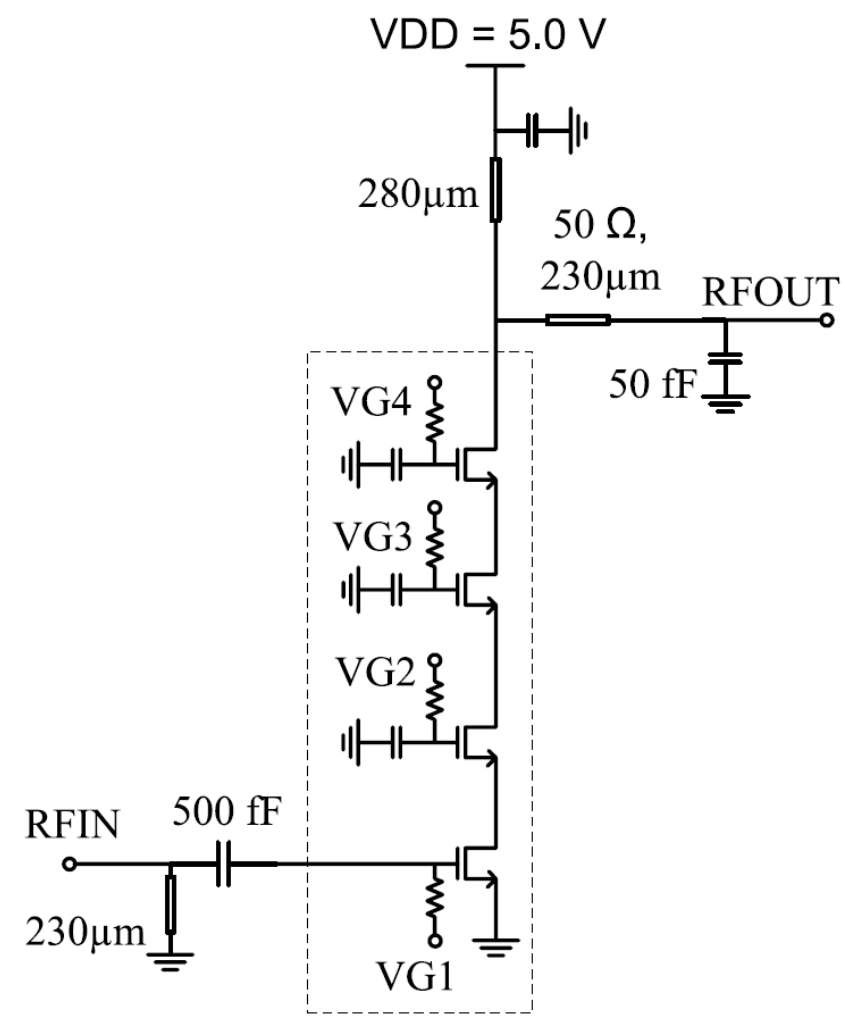

Figure 1.11: 4-stack PA, which employs multi-gate cell layout [21]. 
Table 1.2 below summarizes the performance of the previously reported fully integrated Ka-band $N$-stack PAs implemented in 45-nm RF-SOI.

Table 1.2: Previously reported fully integrated Ka-band $N$-stack PAs in 45-nm RF-SOI.

\begin{tabular}{|c|c|c|c|c|c|c|c|c|c|c|}
\hline Ref \# & Year & $\begin{array}{c}\text { Frequency } \\
\text { [GHz] }\end{array}$ & Topology & $\begin{array}{c}\# \text { of } \\
\text { Stages }\end{array}$ & $\begin{array}{l}\text { Gain } \\
{[\mathrm{dB}]}\end{array}$ & $\begin{array}{c}\mathbf{P}_{1 \mathrm{~dB}} \\
{[\mathbf{d B m}]}\end{array}$ & $\begin{array}{c}\mathbf{P}_{\text {sat }} \\
{[\mathbf{d B m}]}\end{array}$ & $\begin{array}{c}\text { PAE }_{\text {peak }} \\
{[\%]}\end{array}$ & $\begin{array}{c}\text { Area } \\
{\left[\mathbf{m m}^{2}\right]}\end{array}$ & $\begin{array}{c}\text { Results } \\
\text { From } \\
\end{array}$ \\
\hline [16] & 2018 & 28 & 2-Stack & 1 & 13.6 & 16 & 18 & 48.2 & $\begin{array}{c}0.27 \\
\text { (excluding } \\
\text { pads) }\end{array}$ & Measurement \\
\hline$[17]$ & 2017 & 24 & 3-Stack & 1 & 13.1 & 14.2 & 16.2 & 41.5 & 0.41 & Measurement \\
\hline [18] & 2018 & 26.5 & $\begin{array}{l}\text { 2-Stack } \\
\text { PMOS }\end{array}$ & 1 & 10.3 & 16 & 17.8 & 40.7 & 0.37 & Measurement \\
\hline [19] & 2018 & 28 & $\begin{array}{c}\text { 2-Stack } \\
\text { Symmetric } \\
\text { Doherty }\end{array}$ & 1 & 10 & 21.5 & 22.4 & 40 & 0.62 & Measurement \\
\hline [21] & 2016 & 29 & $\begin{array}{c}\text { 4-Stack } \\
\text { Multigate- } \\
\text { cell layout }\end{array}$ & 1 & 13 & 21 & 24.5 & 29 & 0.3 & Measurement \\
\hline $\begin{array}{l}\text { This } \\
\text { work }\end{array}$ & 2019 & 28 & 3-Stack & 1 & 14.1 & 18 & 19.5 & 31.8 & 0.67 & $\begin{array}{c}\text { EM- } \\
\text { cosimulation }\end{array}$ \\
\hline
\end{tabular}




\subsection{Thesis Objectives}

The primary objective of this research work is to assess $45-\mathrm{nm}$ RF-SOI process in terms of its feasibility of implementing 5G mmWave PAs for handsets and small-cell radios. In order to achieve the objective, the research focuses on two main subjects. The first subject is the design and implementation of a unit cell and power transistor cell that offer high power gain at mmWave frequencies. The second subject is the design and implementation of a fully integrated $28 \mathrm{GHz}$ PA that employs transistor stacking as a means of achieving higher RF output power, while overcoming the high knee voltage and low breakdown voltage limitations of advanced node RF-SOI devices. Along with the two main subjects, the layout-driven PA design method will also be presented in this research work.

\subsection{Thesis Contributions}

The major contributions of this research work are as follows. The first is the power transistor cell that achieves a high peak extrinsic $f_{\max }(\sim 280 \mathrm{GHz})$, which translates to a high power gain at mmWave frequencies. Having a high power gain is advantageous not only because it results in a higher PAE of a PA, but also because it relaxes the output power requirement of the driver stage. Furthermore, having a high power gain is also advantageous in terms of die area and overall system power consumption as the number of amplification stages in the transmitter chain can be reduced. Another contribution of this research work is the Ka-band linear PA. Since the use of digital pre-distortion at mmWave frequencies with several hundreds of $\mathrm{MHz}$ to a $\mathrm{GHz}$ bandwidth will be practically difficult, inherently linear PAs are likely to be welcomed and be in high demand. Lastly, the PA implemented in this research work can serve as a building block for a 
fully integrated Doherty PA that can extend the region of high PAE over a wide range of output power.

\subsection{Thesis Organization}

This thesis is organized as follows. Chapter 2 presents the necessary background information on power amplifiers, where various key figures-of-merit (FOM) and the basic concepts of different classes of power amplifiers will be reviewed. This chapter will conclude with reliability considerations of PAs where different types of device breakdown and the ways to prevent them will be reviewed.

Chapter 3 is split into two parts. The first part of the chapter presents an overview of the RF-SOI process where the key RF/mmWave enablement features of the RF-SOI process and the characteristics of PD-SOI devices will be studied. The second part of the chapter presents the design and implementation of transistor cells. The key FOM for active devices that are of importance in PA design will be studied, followed by design considerations of a unit cell. Then, the design and optimization of a unit cell and power transistor cell layout in 45-nm RF-SOI process will be presented.

Chapter 4 begins with an overview of the transistor stacking technique where its principle of operation will be studied. Then, the design and implementation of a fully integrated $28 \mathrm{GHz} 3-$ stack PA will be presented with an emphasis on the layout-driven design method. This chapter will conclude with the EM-cosimulation results of the implemented PA chip and the measurement setup of the chip.

Chapter 5 presents the conclusions of the research work presented in this thesis and suggestions for future work on this topic. 


\section{Chapter 2}

\section{Principles of Power Amplifiers}

A power amplifier (PA) constitutes the last stage of the transmitting system, where its primary role is to drive a load (typically a $50 \Omega$ antenna in wireless communication systems) with the required RF power. PA is typically the most power-consuming component in the whole RF system as it utilizes a substantial amount of DC input power from the power supply in order to produce an amplified replica of the input signal at the output. There are several key figures-ofmerit (FOM) for PA, namely, output power, efficiency, power gain, and linearity. In addition to these classical FOM, wide bandwidth has also become an important design requirement for PAs used in modern wireless communication systems. There are a variety of different classes of PAs, each having its advantages and limitations; hence, their use primarily depends on the type of application the PAs are used or the signal they are subjected to (i.e., type of modulation scheme used). Another important aspect of PA besides the RF performance is the reliability or robustness. Since the devices used in PAs experience high voltage stress, they are more prone to breakdown, and depending on the type of breakdown, the devices may suffer permanent damage or performance degradation over time. 
This chapter presents the necessary background information on PAs, where various key FOM and the basic concepts of different classes of power amplifiers will be reviewed. This chapter concludes with the reliability considerations of PAs where different types of device breakdown and the ways to prevent them will be discussed.

\subsection{Key Figures of Merit (FOM) for PAs}

The key FOM for PAs are output power, efficiency, power gain, and linearity. As will be discussed in this section, some of the FOM are correlated with one another that improvement in one FOM causes degradation in the other. Hence, oftentimes, compromises have to be made on the achievable RF performance.

\subsubsection{Output Power}

The RF output power of a PA can be expressed as shown in (2.1) [23], where $V_{p p}$ and $I_{p p}$ are the peak-to-peak values of the fundamental output voltage and current waveforms, respectively.

$$
P_{R F \_o u t}=\frac{V_{p p} I_{p p}}{8}
$$

Under the premise that the device output capacitance has been resonated out with an output inductor, maximum power can be extracted from the device by presenting an optimum fundamental load resistance $R_{\text {opt }}$ at the device output. The value of $R_{\text {opt }}$ can obtained from (2.2) [23] where $V_{\text {knee }}$ and BVDSS are the knee voltage and drain-source breakdown voltage of the device, respectively while $I_{\max }$ and $I_{\min }$ are the maximum and mimimum drain current, respectively $\left(I_{\min }=0\right)$.

$$
R_{\text {opt }}=\frac{B V D S S-V_{\text {knee }}}{I_{\max }-I_{\min }}
$$


Figure 2.1 shows the optimum load line, which has a slope $-1 / R_{\text {opt }}$. It can be seen that if the quiescent point is selected exactly in the mid-point of the maximum output voltage and current excursion range, and the RF input signal is kept within the cut-off and saturation boundaries, the output voltage and current waveforms can go through their maximum excursions without distortion.

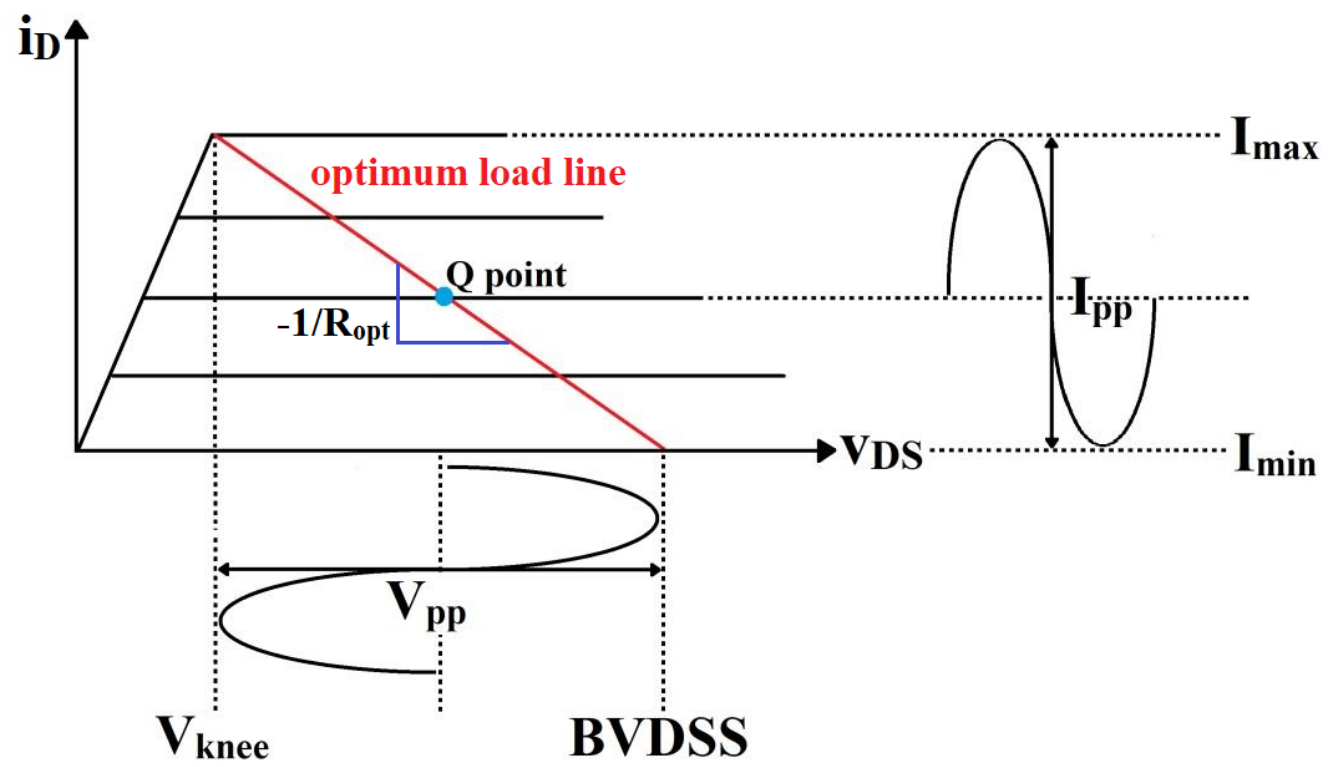

Figure 2.1: Optimum load line and quiescent point that results in maximum output voltage and current excursions (for ideal class-A operation).

Consequently, the maximum RF output power of a PA can be expressed as shown in (2.3) [23].

$$
P_{R F \_o u t, \max }=\frac{\left(B V D S S-V_{\text {knee }}\right)\left(I_{\max }-I_{\min }\right)}{8}
$$

It is important to note that, since the output voltage excursion is strictly limited by $V_{k n e e}$ and BVDSS, which are process parameters, the output current excursion has to be increased in order to maximize the output power of a PA. To this end, PAs employ a large periphery transistor, which results in a small $R_{\text {opt }}$. The small $R_{\text {opt }}$ of a large periphery transistor is advantageous in terms of output power; however, it also limits the achievable performance of a PA. The issues of a large periphery transistor will be considered in some depth in the next chapter. 


\subsubsection{Power-Added Efficiency}

The efficiency of a PA is defined using two metrics, drain efficiency $\eta_{\text {drain }}$ and power-added efficiency (PAE), where the results of both metrics are expressed in percentage terms. Drain efficiency represents how effectively the DC input power drawn from the power supply is converted into RF output power, and it is expressed as the ratio of $P_{R F_{-} \text {out }}$ to $P_{D C}$ as shown in (2.4) [23].

$$
\eta_{\text {drain }}=\frac{P_{R F_{\_} \text {out }}}{P_{D C}}
$$

Drain efficiency, however, is not a very accurate indicator of PA's efficiency as it does not take into account the gain (or, in other words, the RF input power) of a PA. An alternative expression and perhaps a more realistic indicator of PA's efficiency is PAE, which can be expressed as shown in (2.5) [23].

$$
P A E=\frac{P_{R F_{-} \text {out }}-P_{R F_{-} \text {in }}}{P_{D C}}=\eta_{\text {drain }}\left(1-\frac{1}{G}\right)
$$

Contrary to the drain efficiency, PAE takes into account the gain of a PA, and hence reflects the gain compression that occurs at high input drive levels. However, it should be noted that if the gain of a PA is high (i.e., higher than $10 \mathrm{~dB}$ ), PAE is more or less equal to the drain efficiency as the effect of RF input power on efficiency is isolated. Figure 2.2 shows the output power, drain efficiency, and PAE as a function of input power. It can be seen that while drain efficiency keeps on increasing over the entire range of input power, PAE increases for low input power, reaches a peak, then starts to decline at higher input power. The peak PAE typically occurs a few $\mathrm{dB}$ above the 1-dB compression point $P_{1 d B}$. 


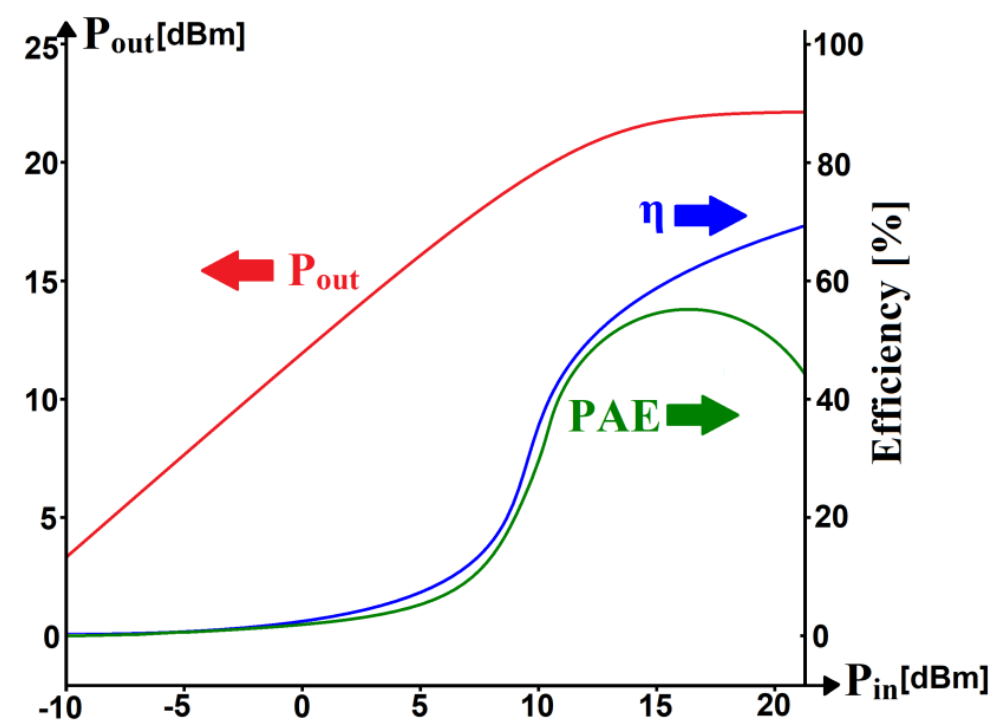

Figure 2.2: Output power, drain efficiency, and power-added efficiency versus input power.

Achieving high efficiency is a crucial task as high efficiency leads to extended battery life for battery-powered devices and less operating costs for base stations (i.e., less costs associated with air-conditioning). Furthermore, maintaining high efficiency over a wide signal dynamic range has become increasingly important in PA design as modern wireless communication uses complex modulated signals that have large envelope variations or high peak-to-average power ratio (PAPR). There are several widespread efficiency enhancement techniques for PAs namely, Doherty, Chireix out-phasing, envelope elimination and restoration (EER), and envelope tracking (ET) [22]. Each of these techniques has its advantages and limitations; hence their use primarily depends on the type of application or transmitter architecture.

\subsubsection{Power Gain}

The power gain of a PA is the ratio of RF output power to RF input power $\left(P_{R F_{-} \text {out }}\right.$ and $P_{R F_{-} \text {in }}$ in watts), and it can be expressed as shown in (2.6) [23]. 


$$
G=\frac{P_{\text {RF_out }}}{P_{\text {RF_in }}}
$$

Contrary to the small-signal amplifiers where the input and output of the amplifier are simultaneously conjugate-matched to the source and load impedances, respectively for maximum gain, PAs typically employ conjugate matching only at the input, while the output is matched to another impedance point referred to as the optimum load impedance $Z_{\text {opt }}$ (or equivalently $\Gamma_{\text {opt }}$ ). $Z_{\text {opt }}$ is an impedance point which, when matched to, results in maximum output power of a PA at high input drive levels; this type matching is known as large-signal power match, and $Z_{\text {opt }}$ is typically found using an iterative search method called load-pull. The basic concepts of the small-signal conjugate match and large-signal power match is graphically illustrated in Figure 2.3 .

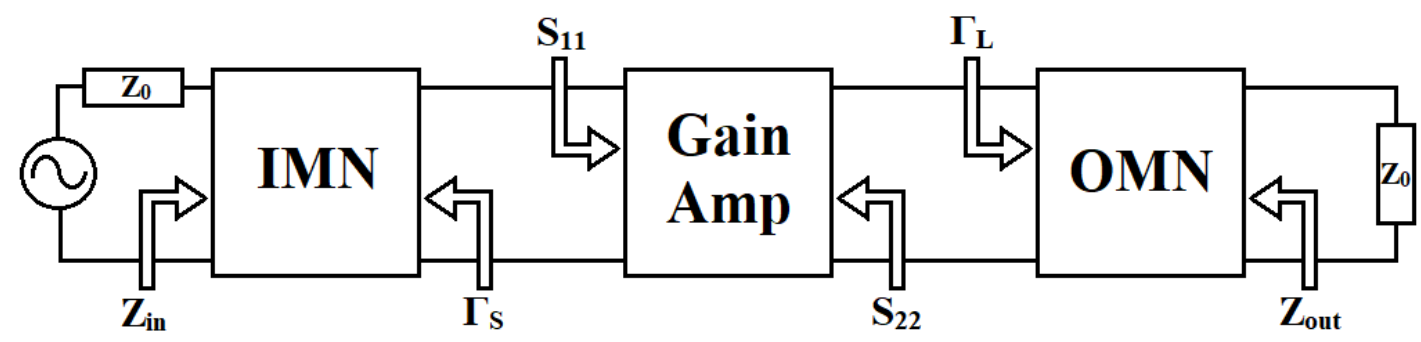

if matched, $Z_{\text {in }}=Z_{0}, \Gamma_{S}=S_{11}{ }^{*}$ and $Z_{\text {out }}=Z_{0}, \Gamma_{L}=S_{22}{ }^{*}$

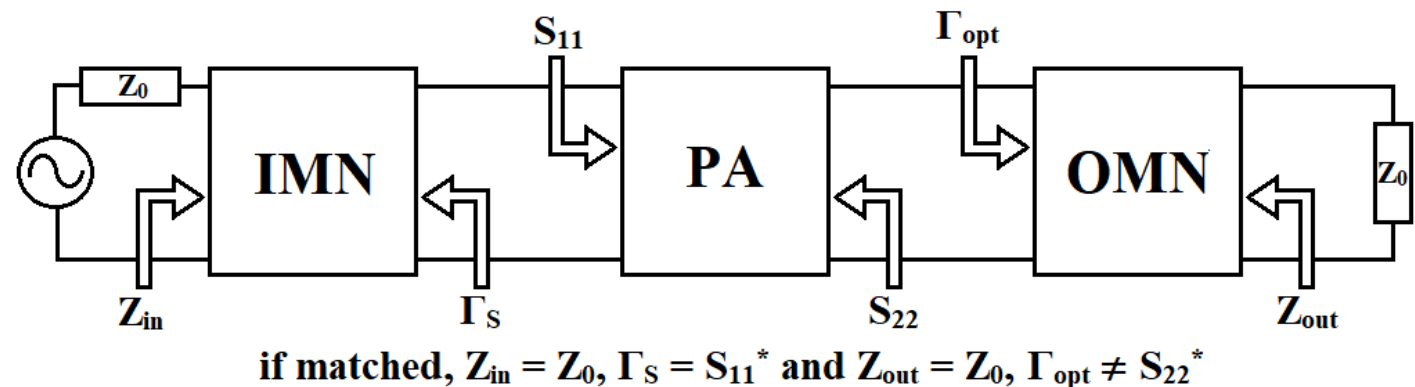

Figure 2.3: Basic concepts of the small-signal conjugate match (top) and large-signal power match (bottom).

The output power of a PA as a function of input power under the two different matching scenarios is shown in Figure 2.4. It can be seen that at high input drive levels, the large-signal 
power match results in a higher output power than the small-signal conjugate match. However, the higher output power from the large-signal power match comes at the expense of losing a bit of small-signal gain; this is, however, an acceptable trade-off as PAs are typically driven into saturation for maximum efficiency.

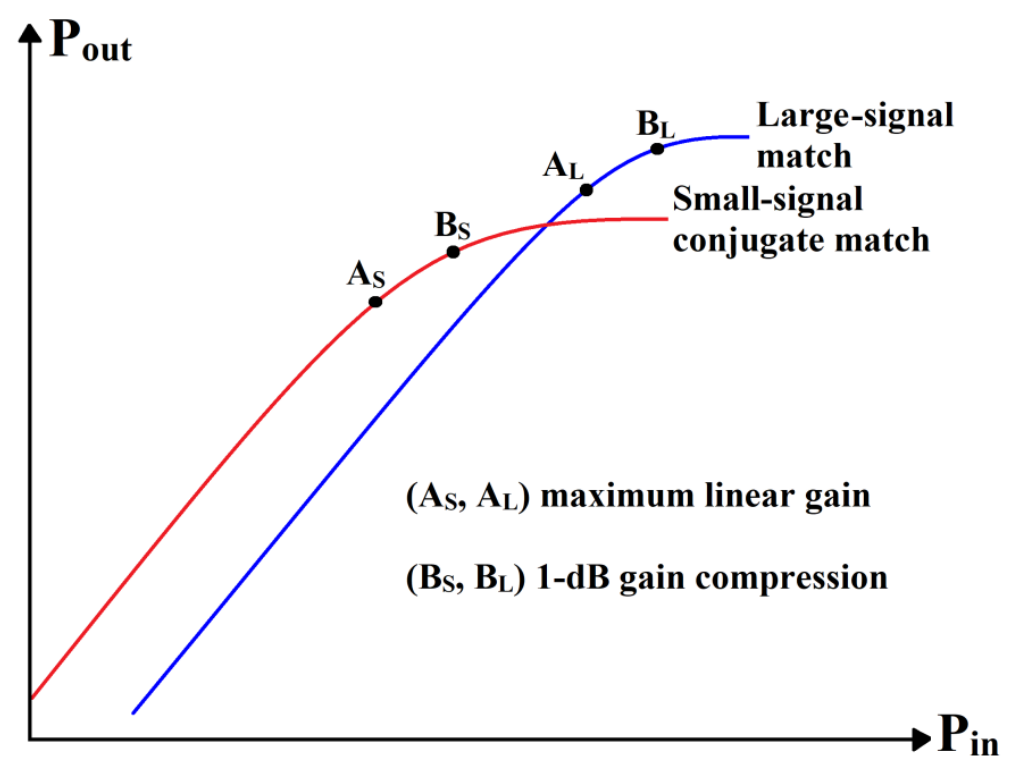

Figure 2.4: Output power of a PA under two different matching conditions, small-signal conjugate match (red) and large-signal power match (blue).

\subsubsection{Linearity}

Linearity is associated with the capability of a PA to amplify the input signal without incurring distortion in the signal waveform. Since PAs are typically operated at or beyond $P_{1 d B}$ where the cutoff and clipping behavior of the devices become prominent, practically all PAs exhibit some degree of nonlinearity. Nevertheless, it is imperative to keep the nonlinearity below an acceptable level.

The nonlinearities of a PA distort both the amplitude and the phase of the signal. The amplitude distortion results in the amplitude of the output signal to differ in shape from the input signal; it is known to be caused by AM-AM conversion, which is associated with gain 
compression and harmonics [23]. The phase distortion, which occurs as the gain of the PA starts to compress, results in an undesired phase shift in the output signal as shown in Figure 2.5; it is known to be caused by AM-PM conversion, which is associated with the voltage-dependent capacitances of the device [24].

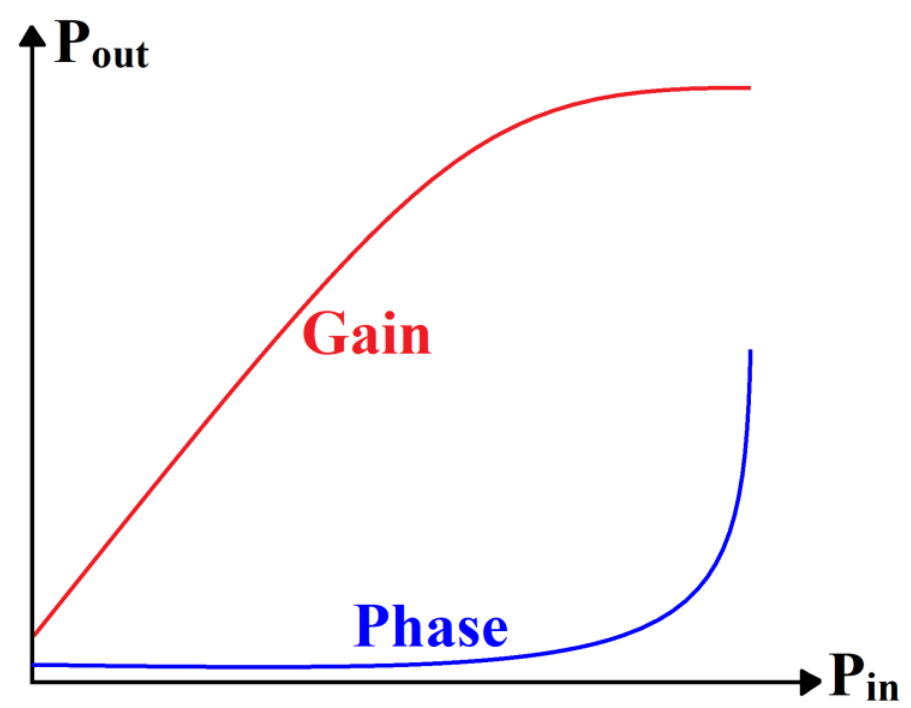

Figure 2.5: Gain compression and phase shift in the output signal.

Modern wireless communication systems impose stringent linearity requirements on PAs as the complex modulated signals require linear amplification. By using more linear PAs, the intermodulation products spreading into the adjacent channels (which is referred to as spectral regrowth) can be reduced, which, in turn, reduces the adverse impact on the adjacent channel's signal quality.

There are several measures that indicate the linearity of a PA, namely, 1-dB compression point $P_{l d B}$, third-order intercept point (IP3), adjacent channel power ratio (ACPR), and error vector magnitude (EVM). $P_{I d B}$ and IP3 are measures of linearity under a single-tone and twotone excitation, respectively, whereas ACPR is a measure of linearity under a modulated signal condition. EVM, although not a direct measure of PA's linearity, is an important linearity measure for digital transmitters whose linearity is largely determined by the linearity of a PA. 
There are several ways to improve the linearity of a PA. One of the easiest ways is to operate the PA at a backed-off power (from the saturated output power) so that the cutoff and clipping behavior of the devices can be avoided. Another way to improve the linearity of a PA is to operate the PA at a higher conduction angle by reducing the quiescent current. The improvement in linearity achieved with these two methods, however, comes at the expense of efficiency degradation. If high linearity is required without compromising the efficiency, external circuits can be employed. The most widespread linearization circuits are feedback, feedforward, and predistortion [23].

\subsection{Classes of PA}

PAs are broadly categorized as either conventional or switch-mode PAs. Conventional PAs are further classified into class-A, $-\mathrm{AB},-\mathrm{B}$, and $-\mathrm{C}$ PAs based on the conduction angle of the PAs, while the switch-mode PAs are further classified into class-E and -F based on the circuit topology. In this section, the basic concepts of different classes of PAs will be presented. It should be noted that there are other classes of PAs in addition to the ones covered in this section; they have been deliberately omitted to limit the scope of this work.

\subsubsection{Class-A, -AB, -B, and -C}

In conventional PAs, a device operates as a voltage-dependent current source (i.e., output current determined by the input voltage); hence, the PAs are classified based on the conduction angle of the device, where the conduction angle is defined as the fraction of a full cycle (in degrees) for which the device conducts currents. Figure 2.6 shows DC-IV curves of a simple Common- 
Source (CS) topology PA on which the load lines for class-A, -AB, -B, and -C operations are overlaid, and also their corresponding output voltage and current waveforms.

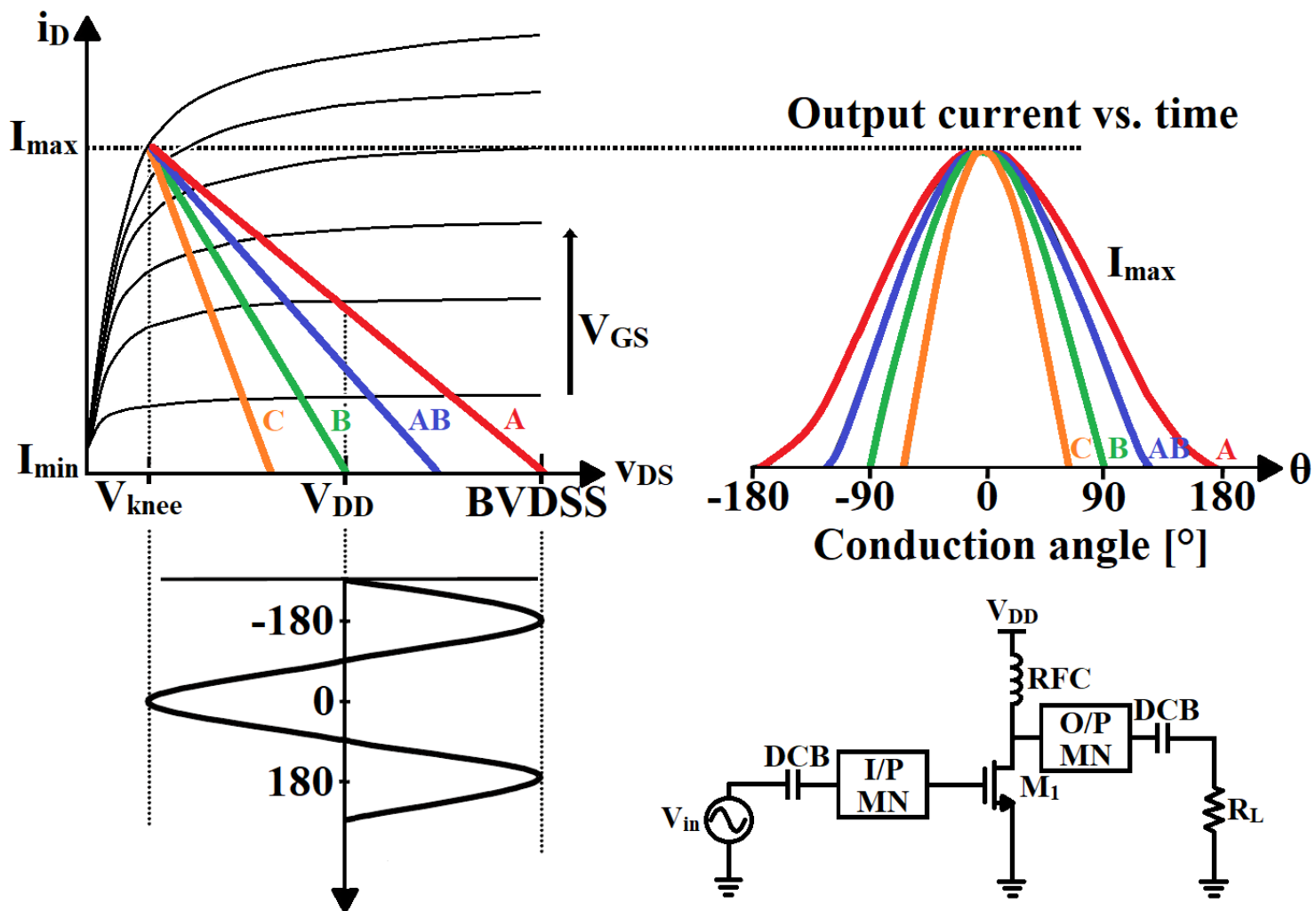

Output voltage vs. time

Figure 2.6: Load lines for different classes of operation and corresponding output voltage and current waveforms.

It can be seen that different classes of operation can be obtained by simply altering the input bias voltage $V_{G S}$ while the output bias voltage is fixed at the power supply voltage $V_{D D}$. In other words, by varying the quiescent current $I_{D}$ in the device, the operating class of the PA can be varied.

For class-A operation, $V_{D S}$ is set to $V_{D D}$, and $V_{G S}$ is set to a value that results in $I_{D}$ to be in the mid-point between $I_{\max }$ and $I_{\min }$. Operating the device at such a quiescent point allows the output current and voltage waveforms to go through their maximum excursions without distortion. Under the simplifying assumption that $V_{\text {knee }}$ is $0 \mathrm{~V}$ and BVDSS is $2 V_{D D}$, the output 
voltage can swing from 0 to $2 V_{D D}$, and the output current can swing from 0 to $I_{\max }$. In class-A operation, the device conducts current for the full cycle of the signal, and hence the conduction angle is $360^{\circ}$. The theoretical maximum drain efficiency that can be achieved in class-A operation is $50 \%$.

Class-AB, -B, and -C operations can be obtained by lowering $V_{G S}$ while $V_{D S}$ is kept at $V_{D D}$. For class-AB operation, $V_{G S}$ is set to any value between class-A operation and device cutoff $\left(V_{G S}>V_{t}\right)$, while for class-B and -C operation, $V_{G S}$ is set to $V_{t}$, and below $V_{t}$, respectively. As $V_{G S}$ is lowered, the device conducts current for a shorter fraction of the full cycle, translating to a smaller conduction angle. However, in principle, the output current can still swing up to $I_{\max }$ even for lower conduction angles. The theoretical maximum drain efficiency that can be achieved in class-AB, -B, and $-\mathrm{C}$ operations are 50 to $78.5 \%, 78.5 \%$, and 78.5 to $100 \%$, respectively.

Figure 2.7 shows the normalized maximum output power of a PA as a function of conduction angle, where the maximum output power of a PA is normalized to the maximum output power of class-A operation. In principle, class-A to class-B operation results in nearly the same maximum output power while the theoretical maximum drain efficiency is higher for lower conduction angles; however, in practice, the achievable output power is lower for lower conduction angles. Class-C operation, on the other hand, results in the highest theoretical maximum drain efficiency, but the maximum output power declines rapidly as the conduction angle decreases. In addition to the output power and efficiency, the gain of a PA reduces for lower conduction angles; this is due to the fact that as the conduction angle becomes smaller, the PA requires a higher input drive for the same output power. 


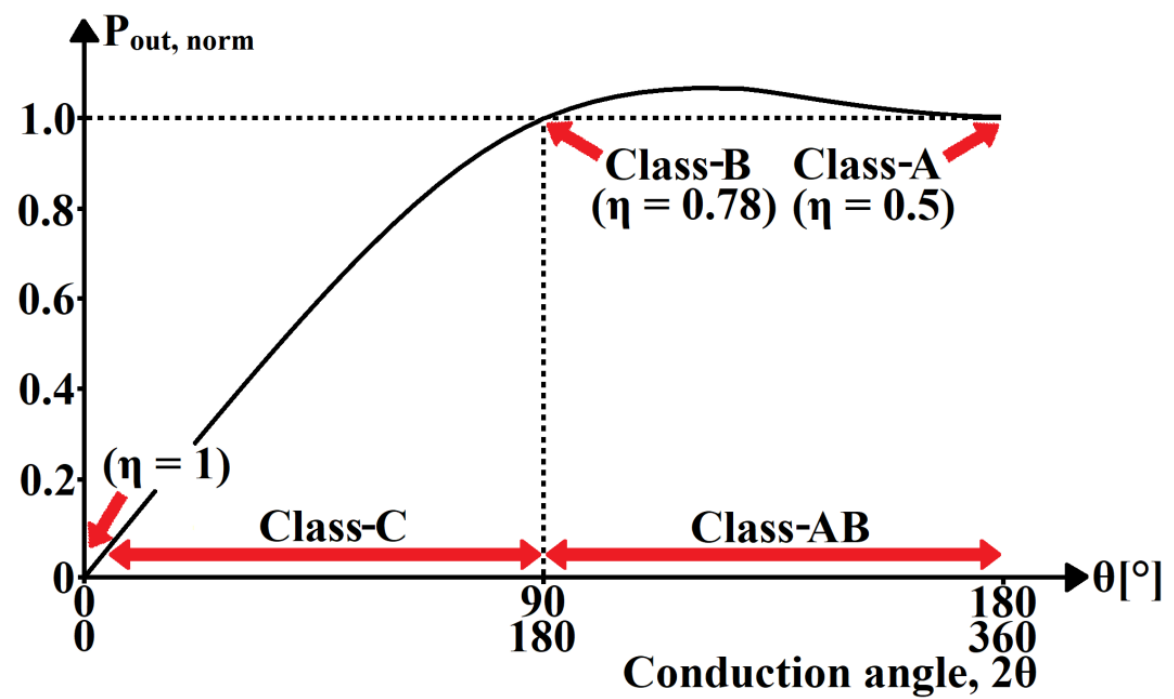

Figure 2.7: Normalized maximum output power versus conduction angle.

The amount of harmonic components present in the drain current is an important indicator of PA's linearity. Figure 2.8 shows the normalized current components as a function of conduction angle where the current components $(\mathrm{n}=1,2,3,4$, and 5) are normalized to the maximum current swing in the device (i.e., $I_{\max }-I_{\min }$ ). It can be seen that for class-A operation, there are no harmonic components in the drain current. However, as the conduction angle becomes smaller, more harmonic components appear in the drain current, indicating the degradation of linearity. Nevertheless, these harmonic components can be prevented from reaching the load by placing a low-pass output matching network. It was described in Section 2.1.2 that the efficiency of a PA is inversely proportional to the conduction angle; this shows that there is a clear trade-off between efficiency and linearity.

Table 2.1 below summarizes the performance of different classes of conventional PAs. While class-C PA offers the highest efficiency, due to its low output power and poor linearity characteristics, it is typically not used as a stand-alone PA in modern wireless communication systems. Class-A PA, on the other hand, is well-suited to work with complex modulated signals due to its excellent linearity; however, with class-A PA, efficiency has to be compromised. 


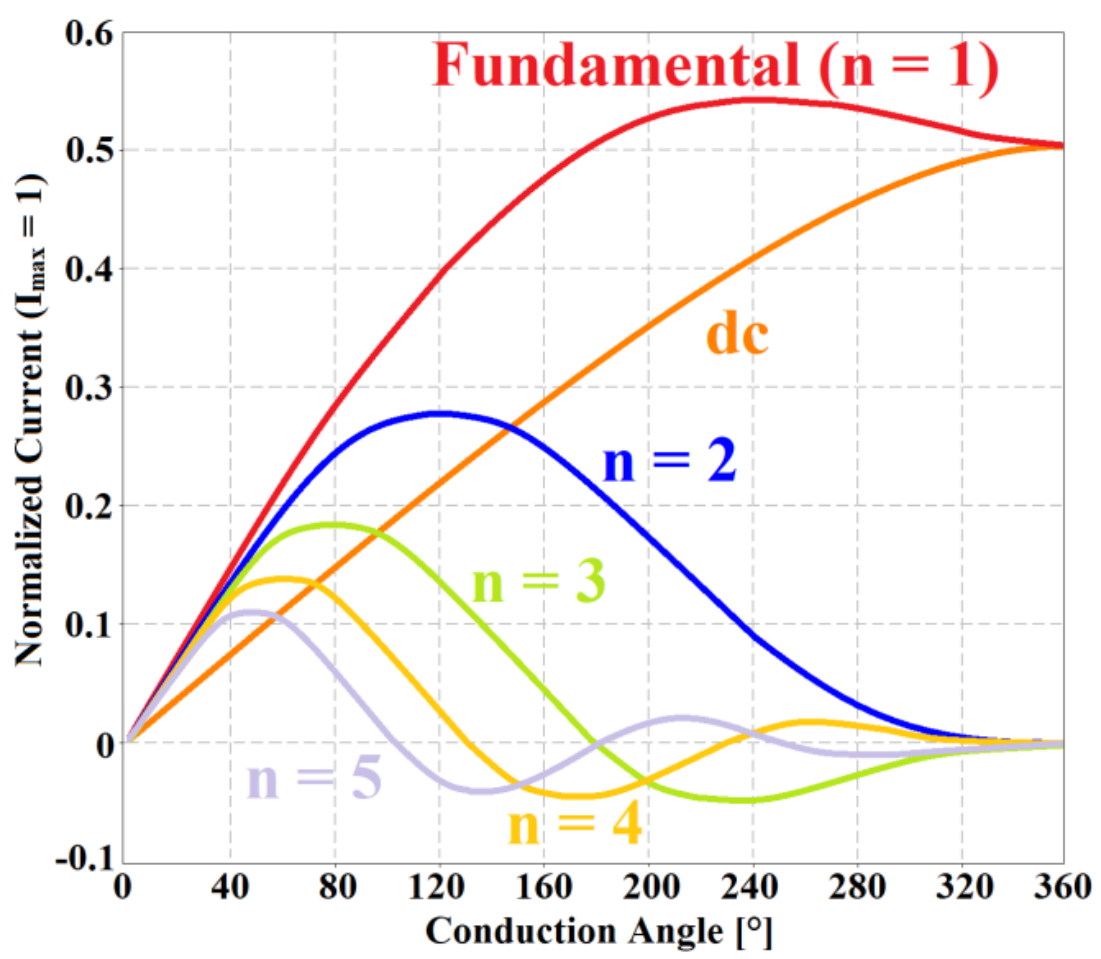

Figure 2.8: Fourier analysis of reduced conduction angle current waveforms.

Table 2.1: Performance comparison of the different classes of conventional PAs.

\begin{tabular}{|c|c|c|c|c|c|}
\hline Class & $\begin{array}{c}\text { Conduction } \\
\text { Angle } \\
{\left[{ }^{\circ}\right]}\end{array}$ & $\begin{array}{c}\text { Normalized } \\
\text { Output Power }\end{array}$ & Gain & $\begin{array}{c}\text { Theoretical } \\
\text { Maximum } \\
\text { Efficiency } \\
{[\%]}\end{array}$ & Linearity \\
\hline A & 360 & 1 & Excellent & 50 & Excellent \\
\hline AB & $360-180$ & $\sim 1$ & Good & $50-78.5$ & Good \\
\hline B & 180 & 1 & Moderate & 78.5 & Moderate \\
\hline C & $180-0$ & $\begin{array}{c}1 \text { at } 180^{\circ}, 0 \text { at } \\
0^{\circ}\end{array}$ & Poor & $78.5-100$ & Poor \\
\hline
\end{tabular}

In practical PAs, the output voltage swing is less than the ideal case ( $0 \mathrm{~V}$ to $\left.2 V_{D D}\right)$ and not sinusoidal due to the finite $V_{\text {knee }}$, and BVDSS. Also, the output capacitance of the device causes the output current to swing below zero. Consequently, the output power of practical PAs is lower than the ideal case. Furthermore, since the matching networks contribute to additional losses in the output power, the efficiency of the practical PAs is much lower than the theoretical maximum. 


\subsubsection{Class-E, and -F}

The efficiency of a PA is fundamentally limited by the power dissipation in the device, which is equal to the product of its drain voltage and current waveforms. Class-E and -F PAs, which belong to the category of switch-mode PAs, achieve very high efficiency (theoretically 100\%) by shaping the drain voltage and current waveforms such that their overlap in time is minimized. Such waveform shaping is carried out by operating the device as an ON-OFF switch (i.e., operating the device either in saturation region or cut-off) and presenting a proper load network at the output of the device. A major drawback of switch-mode PAs is that they are highly nonlinear; hence, unless a substantial linearization is applied, they are unsuited to work with complex modulated signals [10].

Figure 2.9 shows a basic configuration of class-E PA. It is comprised of a device $M_{1}$; an RF choke inductor $L_{R F C}$; a shunt capacitor to ground $C_{1}$; a series $\mathrm{LC}$ resonator formed with $L_{1}$ and $C_{2}$; and a load resistor $R_{L}$. In the load network, the drain-source parasitic capacitance $C_{d s}$ is typically absorbed into $C_{1}$, and the series $\mathrm{LC}$ resonator is tuned to the fundamental frequency so that only the fundamental component reaches the load. Under the following simplifying assumptions:

1) $L_{R F C}$ has a high impedance at the frequency of operation

2) Load network has infinite $Q$

3) $M_{l}$ operates as an ideal switch the values of $C_{1}, C_{2}, L_{1}$, and $R_{L}$ can be chosen such that the drain voltage $v_{D}$ satisfies the ZeroVoltage Switching (ZVS) and Zero-Derivative Switching (ZDS) conditions shown in (2.7) and (2.8) [25].

$$
v_{D}(T / 2)=0
$$




$$
\left.\frac{d v_{D}}{d t}\right|_{T / 2}=0
$$

Satisfying (2.7) and (2.8) ensures that when the switch is $\mathrm{ON}, v_{D}$ is $0 \mathrm{~V}$ while $i_{D}$ is finite, and when the switch is OFF, $i_{D}$ is zero while $v_{D}$ is finite. As illustrated in Figure 2.9, this mechanism results in non-overlapping $v_{D}$ and $i_{D}$ waveforms, which translates to zero power dissipation in the device.
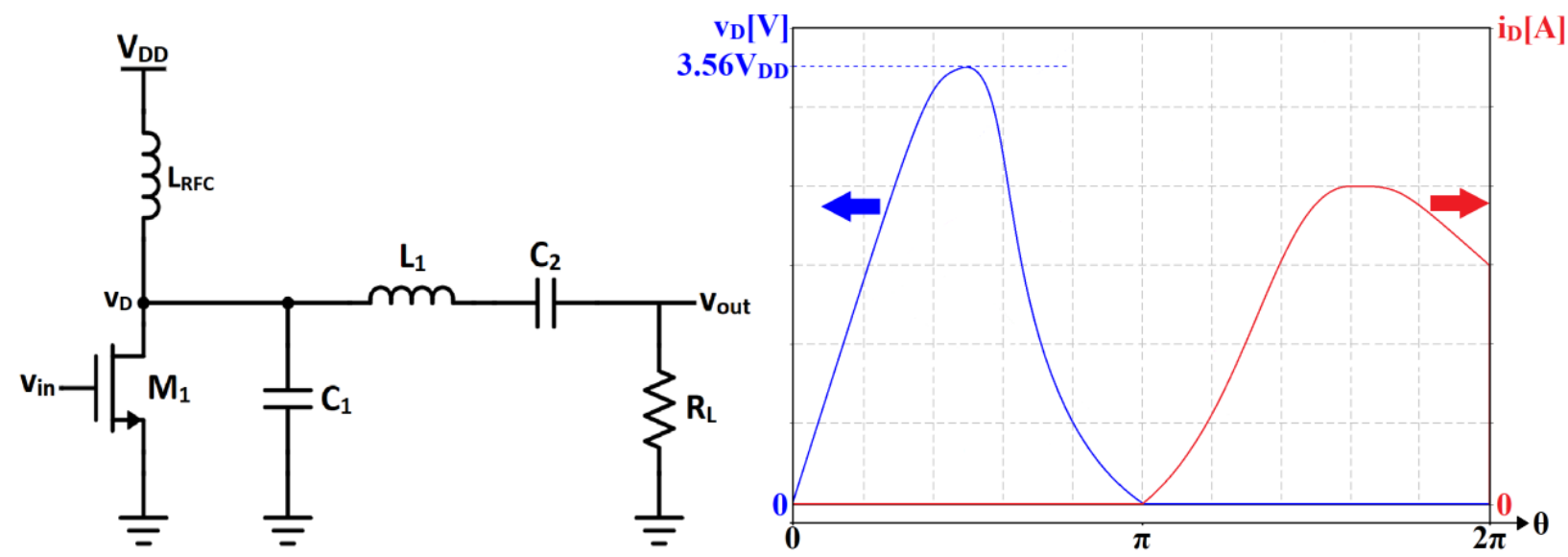

Figure 2.9: Basic configuration of class-E PA and corresponding drain voltage and current waveforms.

In principle, class-E PA can achieve 100\% efficiency while the maximum RF output power is around $78 \%$ of that of a single-ended class-B PA. Nevertheless, in practice, the nonidealities of the actives and passives (i.e., knee voltage $V_{\text {knee }}$, finite on resistance $R_{o n}$, device parasitics, finite inductor $Q$, and finite impedance RF choke inductor) limit the maximum efficiency to $80 \%$ range [23]. A major drawback of class-E PA is that the devices experience a large peak drain voltage of approximately $3.56 V_{D D}$ in the OFF state, which increases the likelihood of device breakdown substantially; hence, the reliability of the PA suffers [26].

Figure 2.10 shows a basic configuration of class-F PA. It is comprised of a device $M_{1}$; an RF choke inductor $L_{R F C}$; a DC blocking capacitor $C_{D C B}$; two parallel LC resonators; and a load 
resistor $R_{L}$. The parallel resonator consisting of $L_{l}$ and $C_{1}$ is typically tuned to the third harmonic frequency in order to present high termination impedance to the third harmonic component; this allows the third harmonic component to be added to the fundamental drain voltage, such that the fundamental drain voltage waveform has a flattened shape, as shown by the blue line in Figure 2.10. Since the drain current $i_{D}$ of class-E PA is a half-wave rectified sinusoid, the overlap between the drain voltage and current waveforms is minimized. Lastly, the resonator consisting of $L_{2}$ and $C_{2}$ is tuned to the fundamental frequency to ensure the output voltage $v_{o}$ is sinusoidal.
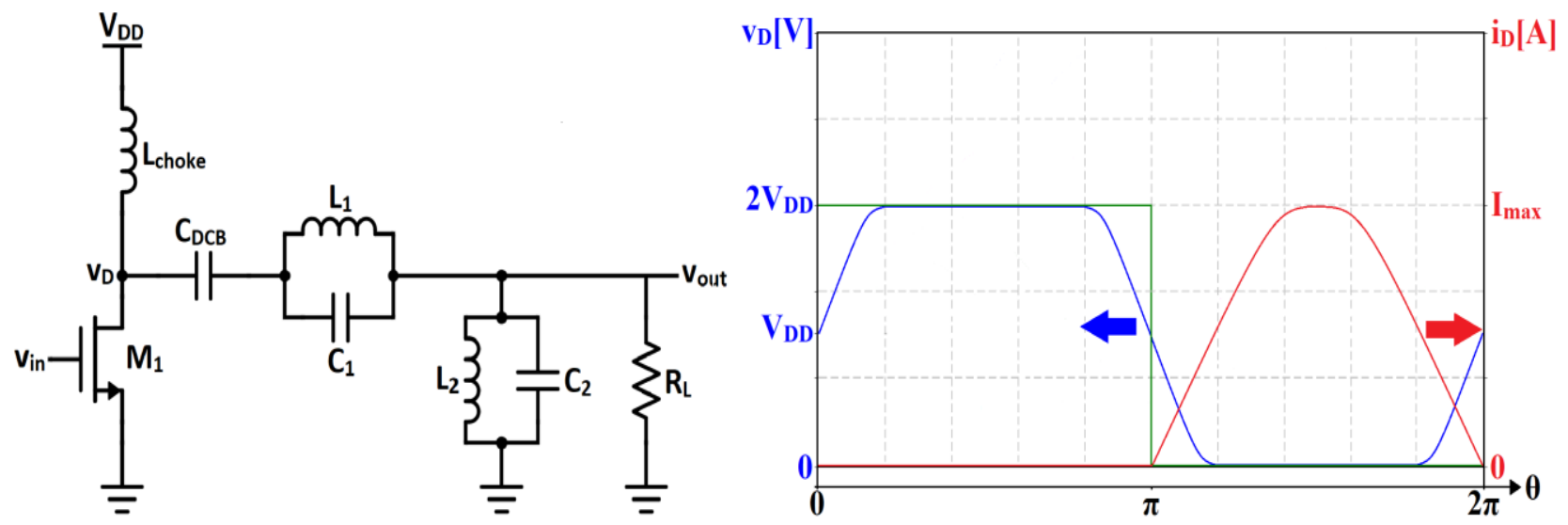

Figure 2.10: Basic configuration of class-F PA and corresponding drain voltage and current waveforms.

In principle, class-F PA produces RF output power and efficiency higher than that of a single-ended class-B PA (output power higher by $27 \%$, and efficiency higher by $10 \%$ ), where the maximum efficiency of class-F PA with third-harmonic peaking is equal to $88 \%$ [23]. In order to improve the efficiency even further, more parallel resonators can be added at the output of the device, or the resonators can be replaced with a quarter-wave transformer; the addition of more resonators results in higher-order odd harmonics components (i.e., $\left.3^{\text {rd }}, 5^{\text {th }}, 7^{\text {th }}, \ldots\right)$ to be added to the fundamental drain voltage, and as a result shape the drain voltage waveform more like a square wave, as shown by the green line in Figure 2.10. If a quarter-wave transformer is used 
instead of the lumped-element resonators, the drain voltage waveform consists of the fundamental component and all odd harmonic components, while the drain current waveform consists of the fundamental component and all even harmonic components; this results in zero voltage and current waveform overlap, leading to $100 \%$ efficiency. However, as in the case of class-E PA, the non-idealities of the actives and passives limit the practical efficiency to lower than the ideal case. Furthermore, the large die area consumption of the additional resonators or the quarter-wave transformer poses a challenge to practical implementation.

\subsection{Reliability Consideration}

Devices used in PAs experience high voltage stress, and as a result they are more prone to breakdown. There are several different types of breakdowns, each having different implications on the device's performance. The gate-oxide breakdown (also called time-dependent dielectric breakdown TDDB), which occurs when the device is subjected to a large $V_{G S}$ or $V_{D S}$, results in instantaneous device failure and may cause permanent damage to the device. On the other hand, the hot carrier injection, which occurs when the device is subjected to simultaneously large $V_{D S}$ and $I_{D}$, results in a degradation of performance over time (e.g., shift in the threshold voltage $V_{t}$ and reduction in the transconductance $\left.g_{m}[43]\right)$. Lastly, the drain-source punch-through, which occurs when a large $V_{D S}$ is applied to the device that is in cutoff $\left(V_{G S}<V_{t}\right)$, causes the gate to lose control of the depletion charge under the channel and results in a rapid increase in $I_{D}$. The drain-source punch-through also results in a degradation of performance over time.

In order to ensure the reliability of a PA, device breakdown must be avoided. There are several ways to prevent device breakdown. For instance, the power supply voltage can be reduced to limit the voltage swing across the device; however, the improvement in reliability 
with this approach comes at the expense of performance degradation (i.e., lower output power, and PAE). Another way to prevent device breakdown is to interconnect or stack several devices such that the voltage across each device is kept within the breakdown voltage limit of a single device. Lastly, thick-oxide devices with higher breakdown voltage can be used; this approach, however, requires compromise on the speed of the device (i.e., lower $f_{T} / f_{\max }$ ).

\subsection{Summary}

In this chapter, various key FOM for PAs and the basic concepts of different classes of power amplifiers were reviewed. The importance of presenting optimum load resistance $R_{\text {opt }}$ at the device output and employing large-signal power match to achieve the maximum RF output power was discussed. In the review of the conventional PAs, it was highlighted that the efficiency of a PA is inversely proportional to the conduction angle of a device, while the linearity of a PA is proportional to the conduction angle of a device. In the review of the switchmode PAs, it was highlighted that very high efficiency can be achieved by operating a device as an ON-OFF switch and presenting a proper load network at the output of the device (such that the overlap of the drain voltage and current waveform is minimized). Lastly, different types of device breakdown were discussed in relation to the reliability of a PA. It was highlighted that while the gate-oxide breakdown results in instantaneous device failure, the hot carrier injection and punch-through results in long-term degradation in the device's performance. This chapter concluded with a brief discussion of techniques that can be used to prevent the device breakdown such as power supply voltage reduction, device stacking, and use of thick oxide devices. 


\section{Chapter 3}

\section{RF-SOI Technology}

As the planar bulk CMOS (hereinafter referred to as "CMOS") technology continued to scale to more advanced nodes, the short-channel effects (SCEs), such as drain-induced barrier lowering, hot carrier effects, punch-through, mobility degradation, and others became severe and harder to control. Hence, in order to control, or to mitigate the SCEs, a combination of various device fabrication techniques (e.g., shallow S/D extension, lightly doped drain (LDD), halo doping, strain engineering, and others) was adopted and has been found to be effective to a certain extent [27]. Nevertheless, the diminishing effectiveness of these techniques with ever-decreasing channel length necessitated a more novel approach to control the SCEs, which has led to the advent of Silicon-on-Insulator (SOI) technology. While SOI has been proven to be effective in mitigating the SCEs, the use of SOI for RF/mmWave ICs was, however, limited as the process was primarily intended for digital ICs; despite the availability of high $f_{T} / f_{\max }$ devices (higher than CMOS), the realization of low-loss transmission lines and high- $Q$ on-chip passives were still challenging due to the low resistivity substrate and digital-optimized BEOL metal stack. However, with persistent efforts, foundries were able to retrofit the SOI process with $\mathrm{RF} / \mathrm{mm}$ Wave enablement features and introduce a variant of the SOI process called RF-SOI. 
This chapter is split into two parts. In the first part of the chapter, an overview of the RFSOI process will be presented where the key $\mathrm{RF} / \mathrm{mmWave}$ enablement features and the characteristics of PD-SOI devices will be studied. In the second part of the chapter, the design and implementation of transistor cells will be discussed. The key FOM for active devices that are of importance in PA design will be studied, followed by design considerations of a unit cell. Then, the design and optimization of a unit cell and power transistor cell layout in 45-nm RFSOI process will be presented.

\subsection{RF-SOI Process Overview}

RF-SOI, being a planar process, leverages the existing CMOS fabrication toolsets and does not require any peculiar processing steps; hence, this leads to substantial savings in both manufacturing time and costs [45]. The fabrication process starts with a high-resistivity (HR) silicon substrate as the base (also called a back-gate) where the resistivity of the substrate is made higher than $1 \mathrm{k} \Omega-\mathrm{cm}$. On top of the HR-substrate, a layer called trap-rich is formed to minimize any conduction at the buried oxide (which is a layer above the trap-rich layer) interface and to suppress the generation of harmonics from the silicon substrate. It has been demonstrated that the presence of the trap-rich layer does not have a significant impact on the DC and RF characteristics of the SOI devices [28]. The combination of the HR-substrate and trap-rich layer leads to significantly lower substrate losses, better noise isolation, higher linearity, and less phase imbalances in the voltage swing [13]. On top of the trap-rich layer, a uniform insulating layer of $\mathrm{SiO}_{2}$, also known as the buried oxide (BOX) layer, is formed to isolate each device electrically from the base substrate (i.e., devices are floating); the presence of the BOX layer essentially 
eliminates the body effect. Finally, the devices are fabricated in an ultra-thin crystalline silicon film on top of the BOX layer.

RF-SOI offers RF/mmWave-optimized BEOL metal stack, which includes thick aluminum and copper top layers. Figure 3.1 shows an example of RF/mmWave-optimized metal stack where the top layer is thick aluminum, and the subsequent two layers are thick copper. The $\mathrm{RF} / \mathrm{mmWave-optimized} \mathrm{metal} \mathrm{stack} \mathrm{also} \mathrm{has} \mathrm{a} \mathrm{larger} \mathrm{distance} \mathrm{to} \mathrm{the} \mathrm{substrate} \mathrm{(so-called} \mathrm{raised}$ metal layers) compared to the digital-optimized metal stack, due to the use of thicker inter-level dielectrics (ILDs); the thicker ILDs are used for reducing the capacitive coupling between the layers and to the substrate $[11,13]$. The presence of HR-substrate and the thick top metal layers allows the realization of low-loss transmission lines and high- $Q$ on-chip passives, making RFSOI a competitive process for $\mathrm{RF} / \mathrm{mm} W a v e$ circuit implementation.

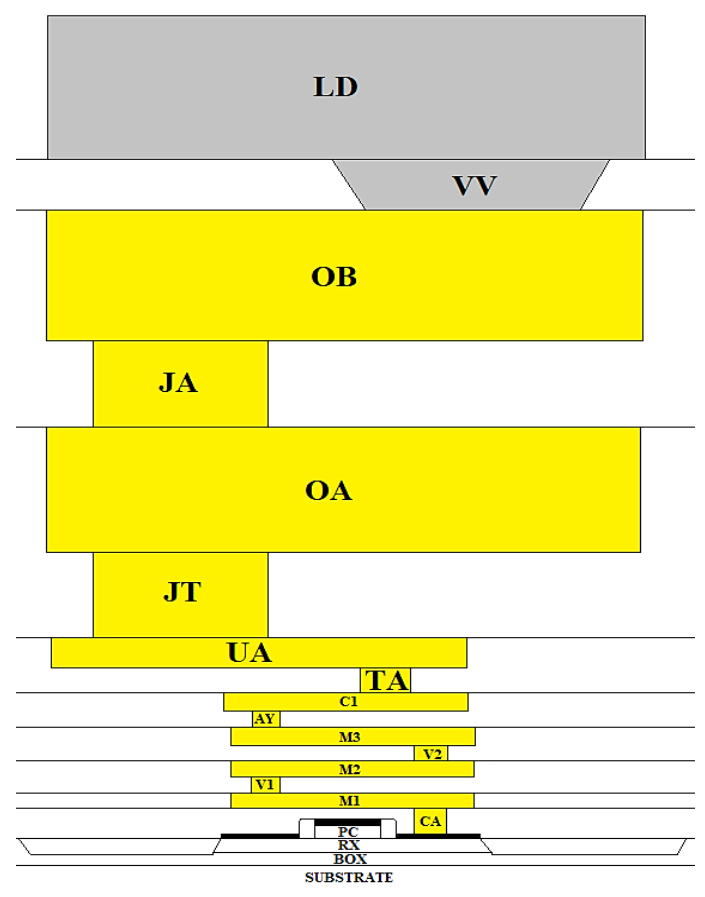

Figure 3.1: RF/mmWave-optimized BEOL metal stack.

RF-SOI offers devices with high $f_{T} / f_{\max }$ as the vast majority of S/D to the substrate parasitic capacitances are effectively reduced by the presence of the BOX layer. Furthermore, the 
enhanced charge carrier mobility achieved with strain engineering also contributes to high $f_{T} / f_{\max }$. Typically, for strain engineering, tensile liner with stress memorization is used with n-type devices, and compressive liner with embedded Silicon Germanium (eSiGe) is used with p-type devices [11].

While RF-SOI offers numerous advantages over CMOS, it also has some drawbacks. Besides the higher wafer cost compared to the CMOS, an undesirable phenomenon called “floating body effect," which alters the device's DC-IV characteristics occurs in RF-SOI devices; the subject of floating body effect will be considered in detail in the next section. Another key drawback of RF-SOI is that it suffers from poor heat dissipation due to the low thermal conductivity of the SOI substrate. In SOI, the devices are isolated laterally by shallow trench isolation (STI), and above and below by ILD and BOX layers, respectively (i.e., devices are enclosed in insulators). Consequently, the devices can heat up substantially with continuous current (e.g., DC or increased leakage currents), and cause what is known as the "self-heating effect (SHE)." SHE is undesirable as it degrades the performance of the device over time (i.e., threshold voltage shift, and reduction in the charge carrier mobility and output current [30]) and can eventually shorten the life span of the device. For the case of PAs, it has been reported that SHE adversely affects the linearity of the PAs and worsens the memory effects [31].

\subsubsection{Partially Depleted (PD) SOI}

RF-SOI offers partially depleted (PD) devices that have SOI film thickness (which is equal to the thickness of the body) of between 50 and 90-nm [32]. The name "partially depleted" comes from the fact that when a channel is created in the device, the body gets partially depleted of the majority carriers as the width of the body is thicker than the width of the depletion region; this 
results in a quasi-neutral region in the body or, simply, a floating body as illustrated in Figure 3.2. When large $V_{D S}$ is applied to the PD-SOI device, the floating body causes a phenomenon called "floating body effect," in which the holes generated from impact ionization near the drain flow to the floating body and get accumulated over time. Consequently, the charged body results in an increased body-source voltage $V_{B S}$, which, in turn, lowers the threshold voltage $V_{t}$. The manifestation of the floating body effect can be seen as a kink and/or increase in the device's output conductance $g_{d s}$ on the DC-IV characteristic curves of the device. The kink effect is undesirable as it degrades the voltage gain and causes excess $1 /$ f noise near the kink region [27]. However, despite the floating body effect, the floating-body PD-SOI devices are still favored for implementing $\mathrm{RF} / \mathrm{mmWave}$ circuits as the kink effect does not significantly degrade the RF performance of the devices, and, in fact, the floating-body PD SOI devices offer the greatest RF performance benefit from the SOI technology.

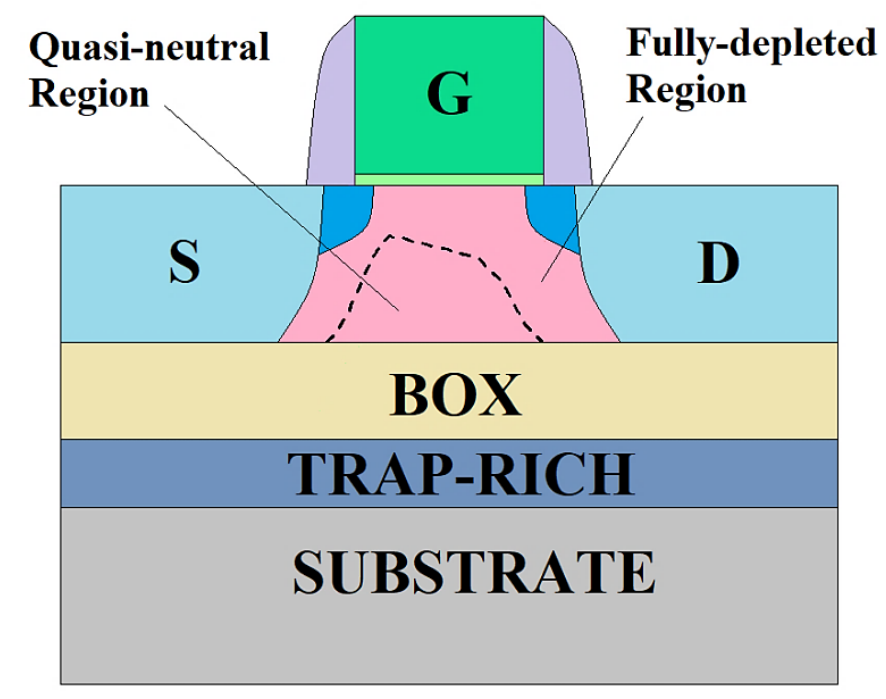

Figure 3.2: Cross-section of a floating-body PD-SOI device.

The floating body effect can be essentially eliminated by forming an electrical body contact. The body-contacted PD-SOI devices offer a better control over the device's DC-IV characteristics, which results in higher transconductance $g_{m}$ and lower $g_{d s}[11]$. Furthermore, the 
body-contacts PD SOI devices offer minimum mismatch between the adjacent devices that arise due to process, voltage, and temperature (PVT) variations; hence, they are well-suited for use in analog circuits (e.g., current mirrors, sense circuits, PLL, comparators). The body-contacted PD SOI devices, however, results in additional wiring capacitances and resistances, which cause degradation of $f_{T} / f_{\max }$ and noise figure of the device. Consequently, the RF performance benefits offered by the SOI technology diminishes with the body-contacted PD-SOI devices. Furthermore, there is also an area penalty with the body-contacted configuration as it consumes more area than the floating-body configuration.

\subsection{Transistor Cell Design}

PAs use transistors whose periphery is substantially larger than those used for other RF active circuits (i.e., LNA, mixer, oscillator, and others). The large periphery transistor, which is generally referred to as a "power transistor," is used so that the transistor has the capability to handle the large current required for producing high output power. A power transistor, however, often limits the achievable RF performance of a PA. For instance, a power transistor has low $f_{\max }$, which translates to less obtainable high-frequency power gain from the device; the low $f_{\max }$ of a power transistor results from the substantial amount of parasitics that arise from its large physical layout. A power transistor also results in a small $R_{\text {opt }}$, which translates to a high impedance transformation ratio up to $50 \Omega$; the high impedance transformation ratio leads to a narrowband output matching unless a multi-section matching network is employed (which is costly due to its large area consumption). Yet another problem with small $R_{\text {opt }}$ is that the matching network becomes physically large, primarily due to the size of the on-chip inductors, and the corresponding high matching losses results in degradation of output power and PAE of a PA. 
Lastly, thermal issues may arise as the power transistor heats up at high output power. A power transistor cell is typically custom-laid out where its large periphery is obtained by increasing the multiplicity of a smaller transistor cell called a "unit cell," and wiring these unit cells together; this process is commonly referred to as scaling. Many of the aforementioned issues of the power transistor can be mitigated by optimizing the unit cell layout, and the way theses unit cells are wired together.

This section will present the design and optimization of transistor cells. The key FOM for active devices that are of importance in PA design will be studied, followed by design considerations of a unit cell. Then, the design and optimization of a unit cell layout in 45-nm RFSOI will be presented. In order to demonstrate the importance of optimizing the transistor cell layout, a performance comparison is made between an unoptimized and optimized unit cell layout. Lastly, the design of a power transistor cell layout, which uses the optimized unit cell layout, will be presented.

\subsubsection{Key FOM for Active Device (relevant to PA Design)}

In RF/Microwave, the two essential FOM that is used to represent the speed of a device is the transit frequency $f_{T}$ and maximum oscillation frequency $f_{\max }$. As a result of continuous technology scaling, devices with very high $f_{T}$ and $f_{\max }$ became available, and these devices have enabled the implementation of high performance mmWave ICs.

The transit frequency is defined as the frequency at which the short-circuit current gain $H_{21}$ falls to unity or $0 \mathrm{~dB}$. As shown in (3.1) [23], it is associated with the transconductance of the device $g_{m}$, the gate-source capacitance $C_{g s}$, and the gate-drain capacitance $C_{g d}$. (3.1) shows that in order to maximize $f_{T}, g_{m}$ has to be maximized while $C_{g s}$ and $C_{g d}$ have to be minimized. 


$$
f_{T}=\frac{g_{m}}{2 \pi\left(C_{g s}+C_{g d}\right)}
$$

Typically, $f_{T}$ is considered an intrinsic device parameter that is optimized during device fabrication stage (i.e., with technology scaling) by reducing the gate length, boosting the effective carrier velocity in the channel, and lowering the series S/D resistances [33].

Maximum oscillation frequency is another parameter used to represent the speed of a device, and it is defined as the frequency at which the maximum available gain (MAG) falls to unity or $0 \mathrm{~dB}$. As shown in (3.2) [23], $f_{\max }$ is associated with $f_{T}$, the gate resistance $R_{g}$, and $C_{g d}$.

$$
f_{\text {max }}=\sqrt{\frac{f_{T}}{8 \pi R_{g} C_{g d}}}
$$

$f_{\max }$ takes into account the extrinsic layout parasitics (which arise from interconnects, contacts, vias, and others) that are added to the intrinsic device core. For PA design, $f_{\max }$ is a more meaningful parameter than $f_{T}$, since power gain is of more concern than current gain. Furthermore, it is important to maximize $f_{\max }$, since the desired RF performance can be achieved more readily with devices that have high $f_{\max }$. In nano-scale devices, however, $f_{\max }$ is susceptible to degradation as the polysilicon gates of the nano-scale devices are very resistive. Also, at $\mathrm{RF} / \mathrm{mmW}$ ave frequencies, the effects of layout parasitics become more severe, and contribute significantly to the degradation of $f_{\max }$. Hence, in order to maximize $f_{\max }$ of a device, the unwanted layout parasitics (primarily $R_{g}$, and to a lesser extent, $C_{g d}$ ) have to be minimized.

As a side note, an interesting finding with respect to $f_{T}$ and $f_{\max }$ is that although different technology nodes have distinct peak $f_{T}$ and $f_{\max }$ values, due to constant-field scaling, the current densities at which peak $f_{T}$ and $f_{\max }$ occur are the same irrespective of the technology nodes. It has been demonstrated across the different technology nodes that peak $f_{T}$ and $f_{\max }$ occur at $\sim 0.3$ $\mathrm{mA} / \mu \mathrm{m}$ and $\sim 0.2 \mathrm{~mA} / \mu \mathrm{m}$, respectively [11]. 


\subsubsection{Unit Cell Design Considerations}

The first thing to consider when designing a unit cell with a fixed width is the gate resistance. As shown by (3.2), in order to maximize $f_{\max }$, the gate resistance $R_{g}$ has to be minimized. $R_{g}$ is comprised of intrinsic and extrinsic components where the intrinsic component is determined by the polysilicon gate of the intrinsic device core, and the extrinsic component is determined by the metal wiring added to the intrinsic device core. For a device that has one gate finger of width $W_{F}$, the intrinsic gate resistance $R_{g_{\text {_in }}}$, which accounts for the distributed $R C$ nature of the polysilicon gate can be expressed as shown in (3.3) [11], where $R_{\text {poly }}$ is the polysilicon sheet resistance, $y=1$, 2 is the number of gate contacts, and $L$ is the channel length.

$$
R_{g_{-} i n t}=\frac{R_{p o l y} W_{F}}{3 y^{2} L}
$$

In order to reduce $R_{g \text { int }}$ while keeping the total gate width the same, the single large finger can be split into $n$ smaller fingers and connected in parallel (this is equivalent to connecting $n$ identical devices in parallel); this results in a reduction of $R_{g_{-} \text {int }}$ by a factor of $n^{2}$. Furthermore, by using double gate contacts $(y=2), R_{g_{-} \text {int }}$ can be further reduced by a factor of 4 . The use of double gate contacts results in an increase in the gate capacitance $\left(C_{g}=C_{g s}+C_{g d}\right)$; however, since the reduction in the intrinsic gate resistance is more dominant than the increase in the gate capacitance, improvement in $f_{\max }$ can be achieved [34]. Figure 3.3 shows peak intrinsic $f_{\max }$ as a function of number of gate fingers for a $16 \mu \mathrm{m}$ unit cell realized with 40-nm floating-body PDSOI devices available in the $45-\mathrm{nm}$ RF-SOI process. It should be mentioned that the $16 \mu \mathrm{m}$ unit cell was realized with a parameterized cell provided in the 45-nm RF-SOI PDK to simply demonstrate the effect number of gate fingers has on $f_{\max }$; hence the extrinsic $R_{g}$ component, which would normally exist in an actual unit cell layout was not taken into account in the simulation. It can be seen from Figure 3.3 that as the number of gate fingers increases from 8 to 
32 (or equivalently, as the unit gate width (UGW) decreases from $2 \mu \mathrm{m}$ to $0.5 \mu \mathrm{m}$ ), the peak intrinsic $f_{\max }$ increases from $235.4 \mathrm{GHz}$ to $418 \mathrm{GHz}$, which translates to $77.5 \%$ improvement; this clearly shows that by having a higher number of gate fingers (or equivalently, using a smaller UGW) for a fixed width unit cell, the intrinsic $f_{\max }$ can be improved significantly as a result of substantial reduction in $R_{g_{-} \text {int }}$.

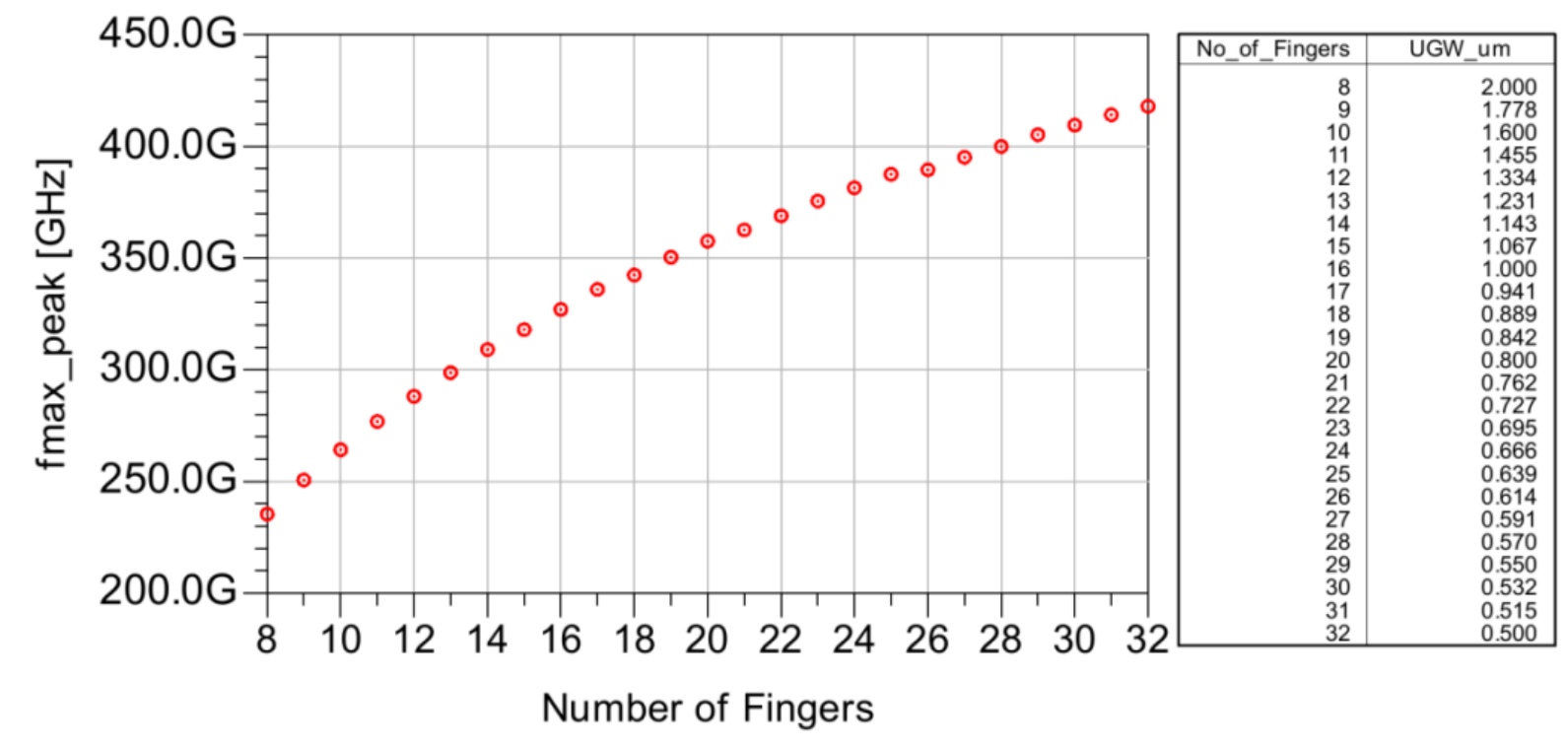

Figure 3.3: Peak intrinsic $f_{\max }$ versus number of gate fingers for $16 \mu \mathrm{m}$ unit cell.

From (3.3) and Figure 3.3, it may seem that using as many gate fingers as possible for a fixedwidth unit cell is advantageous for reducing $R_{g}$. However, in reality, there is a hard limit on the minimum and the maximum number of gate fingers a unit cell can have as the UGW cannot be set to a value outside the $\min / \max$ range allowed by the process. Furthermore, in an actual unit cell layout, increasing the number of gate fingers beyond a certain value results in an increase in $R_{g}$ rather than a reduction; this is because the extrinsic component of the gate resistance $R_{g_{-} \text {ext }}$, which arises from wiring all the gate fingers in parallel increases more dominantly than the decrease in the intrinsic gate resistance [35]. In addition to the effect on the gate resistance, the metal wiring also results in an increase in the parasitic capacitances (i.e., gate to S/D contacts 
capacitances), which contribute to $f_{\max }$ degradation. Consequently, it is crucial to optimize the transistor cell layout such that these unwanted parasitics are minimized. For the unit cell layouts that will be presented in the next section, $1 \mu \mathrm{m}$ was chosen as the UGW for the following three reasons.

1) Intrinsic $f_{\max }$ is considerably higher than $10 \times f_{o}$ (i.e., $>280 \mathrm{GHz}$ ).

2) Intrinsic device core has a width to height ratio of 1:6, and hence the rotational error is small.

3) Being a whole number rather than a rational number minimizes the errors in the actual fabricated width.

\subsubsection{Unit Cell Layout Optimization}

In order to demonstrate the importance of optimizing the transistor cell layout, two different versions of $16 \mu \mathrm{m}$ unit cell layout were created using 40-nm floating-body PD-SOI devices available in the 45-nm RF-SOI process. The top layout in Figure 3.4 shows the default unoptimized unit cell, and the bottom layout shows the custom-designed optimized unit cell (the view of the unoptimized unit cell layout was zoomed in compared to the view of the optimized unit cell in Figure 3.4). UGW of $1 \mu \mathrm{m}$ and double gate contacts were used for both unit cells. Furthermore, two dummy gates were added at the top and bottom of the unit cells to mitigate the boundary dependent etching. 


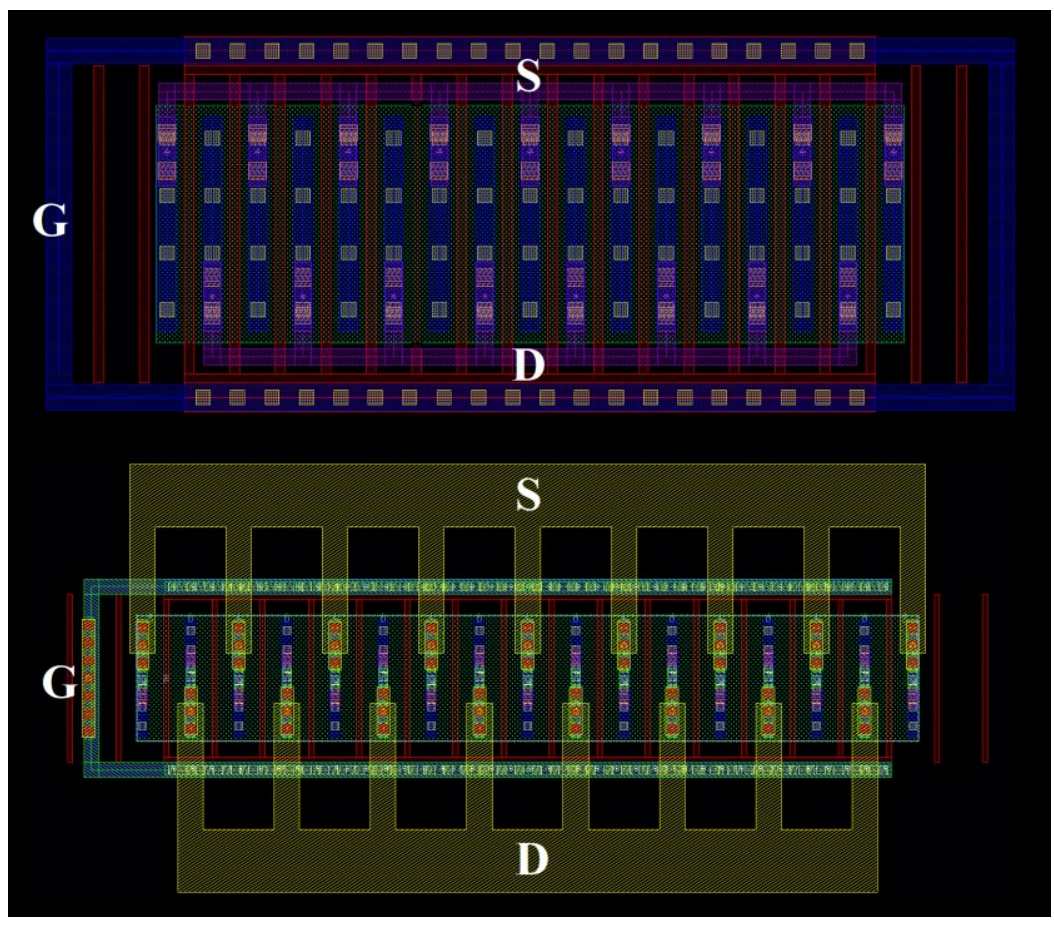

Figure 3.4: Unoptimized (top) and optimized (bottom) $16 \mu \mathrm{m}$ unit cell layout.

In the unoptimized unit cell layout, the gate poly pitch in the intrinsic device core was set to a regular pitch, and the wiring of the gates, sources, and the drains were done using the lowest two metal layers, M1 and M2. In advanced node RF-SOI (or CMOS) process, the lower metal layers allow very small line width and fine wire pitch at the expense of higher sheet resistance; hence, they are typically used for very short wiring within or near the device. In the optimized unit cell layout, the gate poly pitch in the intrinsic device core was set to a relaxed pitch as it has been demonstrated that the relaxed gate poly pitch results in an improved $f_{T}$ due to lower gate to $\mathrm{S} / \mathrm{D}$ contact capacitances, and higher $g_{m}$ from enhanced stress response of the device [36]; however, the improvement in $f_{T}$ comes at the expense of more area consumption. While the thermal aspects of the transistor cell will not be discussed in this work, it is important to note that the number of gate fingers and the gate finger pitch play an important role in determining the maximum temperature rise in the transistor. In addition to using a ring-shaped polysilicon gate 
with double gate contacts, M1-M3 stacked metal was used for the gate in order to reduce the extrinsic gate resistance that arise from wiring. For the sources and drains, the staircase routing technique [37] was employed to minimize the capacitive coupling between the source and drain metals on the same layer, thereby reducing the drain-source capacitance $C_{d s}$. Finally, the sources and drains were wired together using metal strips on $\mathrm{C} 1$ layer, which has relatively lower sheet resistance than the bottom three layers (M1 to M3).
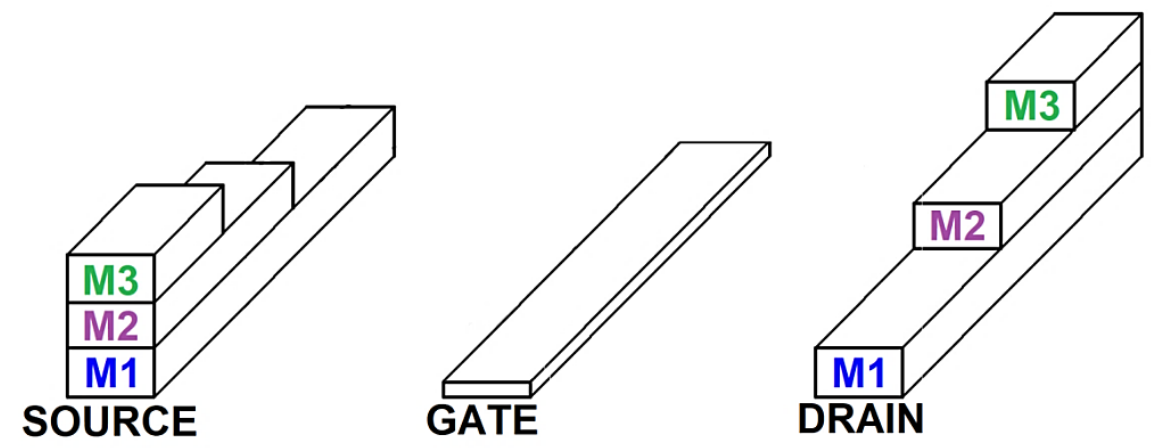

Figure 3.5: Staircase routing to minimize source-drain capacitive coupling [37].

In order to demonstrate the effectiveness of layout optimization, $R C$ parasitic extraction (PEX) simulation was run on both unit cell layouts using Mentor Graphic's Calibre xRC tool embedded within the Cadence Virtuoso environment. The PEX simulation creates an instance that contains the parasitic resistances and capacitances extracted from the physical layout of the unit cell (from polysilicon layer up to $\mathrm{C} 1$ layer); the created PEX unit cell instance can then be imported into a schematic test bench and be used just like an ordinary schematic instance. Using the PEX unit cell instance, S-parameter simulation was run, and the resulting S-parameters were converted into Y-parameters. Then, using (3.4) to (3.9) [34], the high-frequency small-signal equivalent circuit parameters shown in Figure 3.6 were computed where the results are summarized in Table 3.1. The computed small-signal equivalent circuit parameters account for both intrinsic and extrinsic components of the unit cell layout. Nevertheless, it is important to 
note that the high frequency small-signal equivalent circuit model shown in Figure 3.6 is a simplified model, which does not include all the high-frequency elements that are present in the transistor.

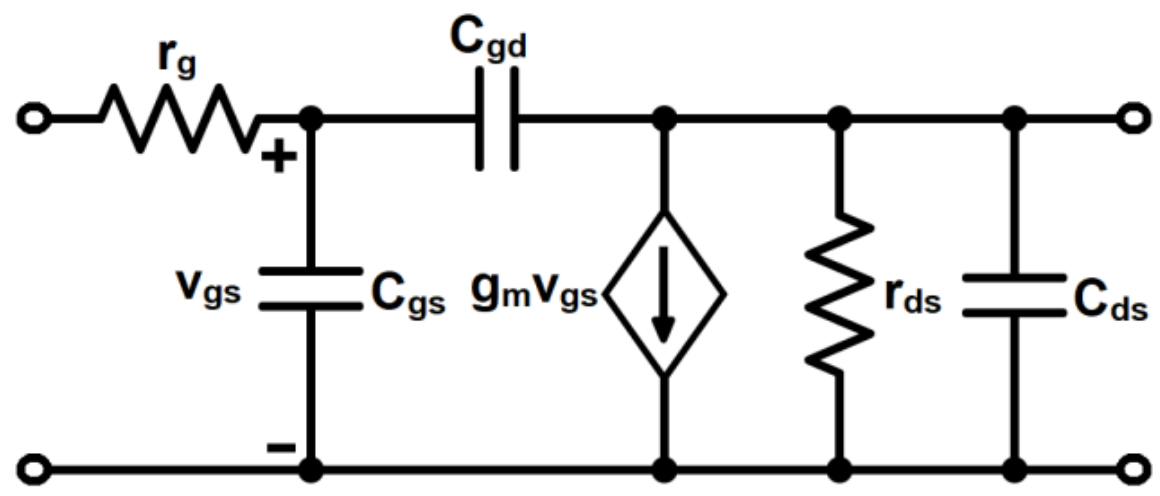

Figure 3.6: High-frequency small-signal equivalent circuit of a transistor.

$$
\begin{gathered}
C_{g s}=\frac{\operatorname{Im}\left(Y_{11}+Y_{12}\right)}{\omega} \\
C_{g d}=-\frac{\operatorname{Im}\left(Y_{12}\right)}{\omega} \\
C_{d s}=\frac{\operatorname{Im}\left(Y_{22}+Y_{21}\right)}{\omega} \\
g_{m}=\operatorname{Re}\left(Y_{21}\right) \\
r_{d s}=\frac{1}{\operatorname{Re}\left(Y_{22}\right)} \\
r_{g}=\frac{\operatorname{Re}\left(Y_{11}\right)}{\left(\operatorname{Im}\left(Y_{11}\right)^{2}\right.}
\end{gathered}
$$

Table 3.1: Computed small-signal equivalent circuit parameters (at $28 \mathrm{GHz}$ ) of the unoptimized and optimized $16 \mu \mathrm{m}$ unit cell.

\begin{tabular}{|c|c|c|}
\hline & $\begin{array}{c}\text { Unoptimized Unit Cell } \\
\mathbf{1 6} \boldsymbol{\mu \mathbf { m }}\end{array}$ & $\begin{array}{c}\text { Optimized Unit Cell } \\
\mathbf{1 6} \boldsymbol{\mu \mathbf { m }}\end{array}$ \\
\hline $\boldsymbol{g}_{\boldsymbol{m}}[\mathbf{m S}]$ & 21 & 22 \\
\hline $\boldsymbol{C}_{g s}[\mathbf{f F}]$ & 9.9 & 10.0 \\
\hline $\boldsymbol{C}_{\boldsymbol{d}}[\mathbf{f F}]$ & 2.7 & 1.6 \\
\hline $\boldsymbol{C}_{\boldsymbol{g} d}[\mathbf{f F}]$ & 5.0 & 5.0 \\
\hline $\boldsymbol{r}_{\boldsymbol{d s}}[\mathbf{\Omega}]$ & 262.9 & 252.3 \\
\hline $\boldsymbol{r}_{g}[\mathbf{\Omega}]$ & 10.2 & 8.6 \\
\hline
\end{tabular}


As can be seen from Table 3.1, while $C_{g d}$ remained the same for both layouts, the optimized unit cell layout resulted in a $15.7 \%$ lower gate resistance than the unoptimized unit cell layout. Furthermore, the optimized layout resulted in $42 \%$ lower $C_{d s}$ than the unoptimized layout attributed to the staircase drain/source routing. In order to see the effect of lower gate resistance on $f_{\max }, f_{\max }$ as a function of drain current density was plotted for both unit cells as shown in Figure 3.7. It can be seen that while peak $f_{\max }$ of both unit cells occurred at the same current density $(0.35 \sim 0.4 \mathrm{~mA} / \mu \mathrm{m})$, the peak $f_{\max }$ of the optimized unit cell was higher than that of the unoptimized unit cell by $17.6 \%$ (395.8 GHz to $336.5 \mathrm{GHz}$ ). This result clearly shows that $f_{\max }$ is sensitive to layout parasitics and hence it is critical to optimize the transistor cell layout to maximize $f_{\max }$. It may seem that the improvement is marginal as demonstrated with the unit cells in this section; however, it should be noted that the improvement in $f_{\max }$ becomes more evident as the size of the transistor cell grows.

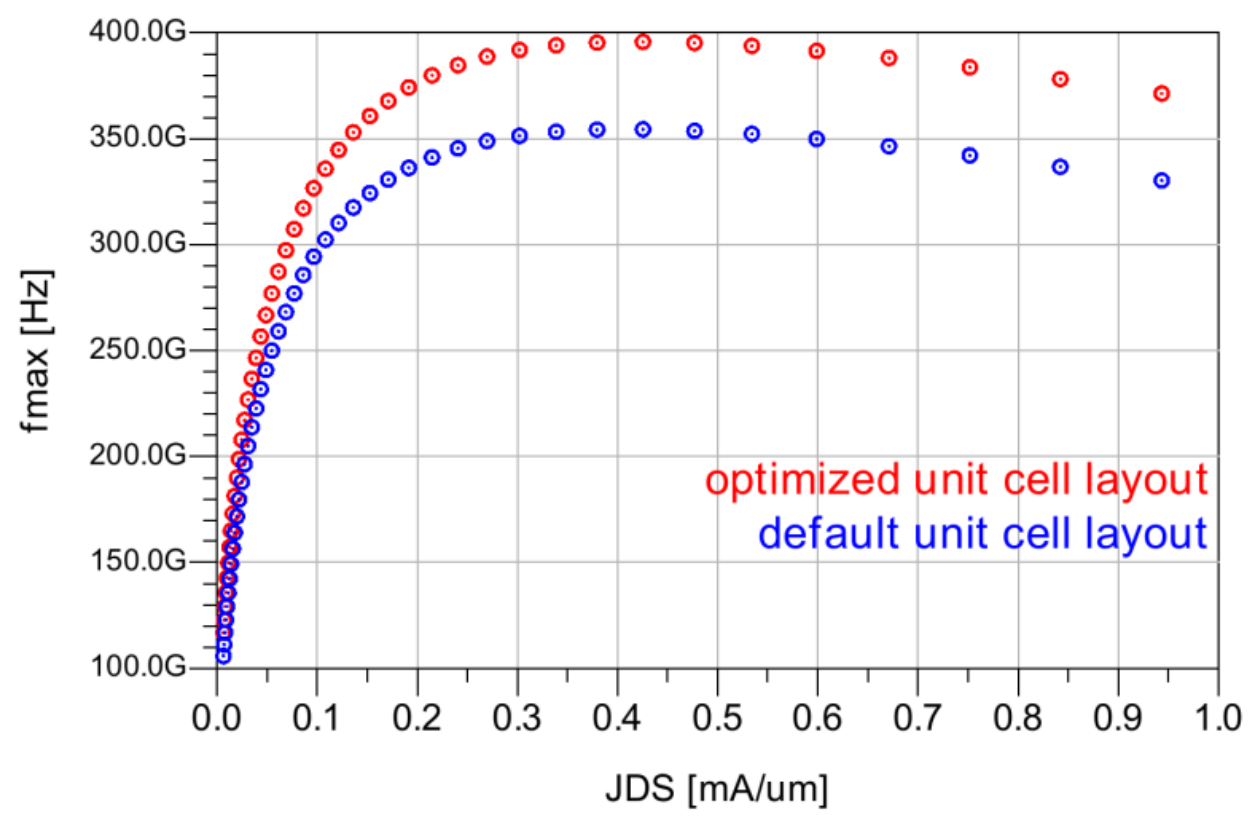

Figure 3.7: Extrinsic $f_{\max }$ versus drain current density for optimized and unoptimized $16 \mu \mathrm{m}$ unit cell. 


\subsubsection{Power Transistor Cell Layout}

A power transistor cell is realized by increasing the multiplicity of a unit cell and wiring the unit cells together. As shown in Figure 3.8, there are several different styles of power transistor cell layout, namely, in-line [11], round-table [38], and zipper [39].

In the in-line style, the multiplicity of a unit cell is increased by placing the unit cells consecutively in a direction perpendicular to the gate orientation. Then the gates, drains, and the sources of the unit cells are strapped together with long metal strips to form global gate, drain, and source nodes. Tapered lines are commonly used at the input and output of the power transistor to mitigate an abrupt impedance transformation. The global source node can be directly connected to the ground plane to realize a common-source configuration. The in-line style is very simple in terms of its layout complexity; however, it is inefficient in terms of area usage and heat distribution as its shape becomes very long for a large periphery transistor. Furthermore, due to its long shape, phase delays can occur at the gate fingers, which can result in PAE degradation of a PA.
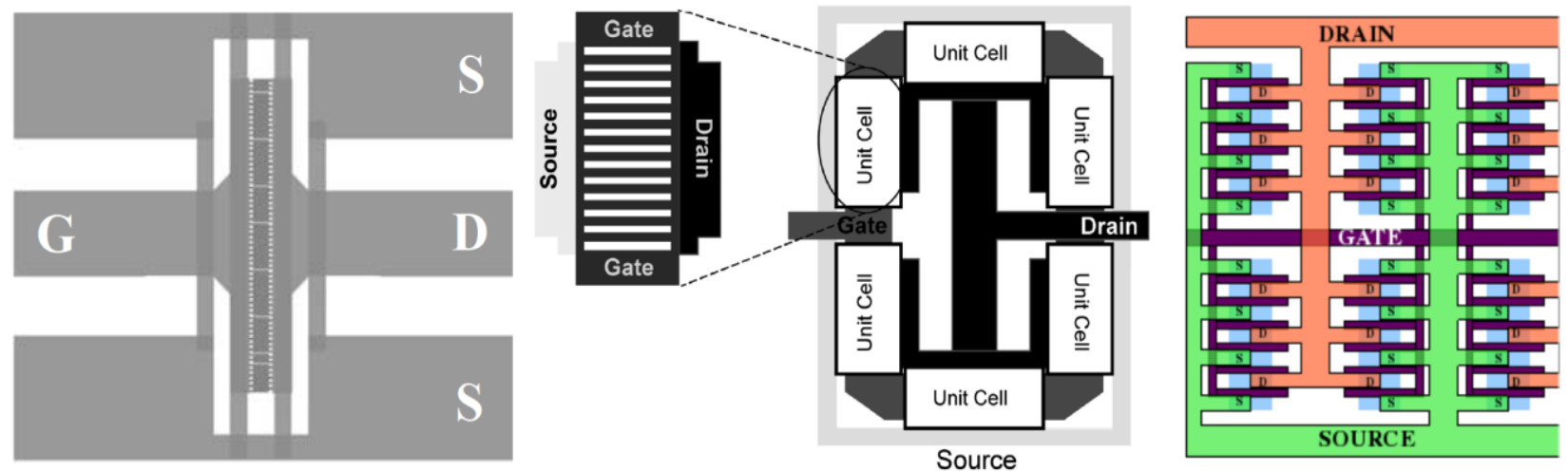

Figure 3.8: Different styles power transistor cell layout; in-line (left), round-table (middle), zipper (right) [11, 38, 39].

A better way to realize a power transistor cell is to wire the unit cells with a pattern or in the form of an array. For instance, in the round-table style, the multiplicity of a unit cell is 
increased by placing the unit cells in a circular manner. The gate contacts at the top and bottom of each unit cell facilitate connections with the adjacent unit cells. The global source and drain nodes are formed by wiring together the sources and drains of the unit cells at the outside and inside of the power transistor cell, respectively. It has been demonstrated that for the same size transistor, the round-table stlye results in a lower gate resistance than the in-line style [38]. Nevertheless, one critical drawback of the round-table style is that it requires the gates of the unit cells to be oriented both in the horizontal and vertical directions; hence, this style of layout cannot be used in processes where only one gate orientation is allowed.

In the zipper style, the multiplicity of a unit cell is increased by placing the unit cells in the form of an array. Then, the global gate, drain, and source nodes are formed by interconnecting the unit cells in a way that resembles a zipper. The zipper style is more area efficient and thermally robust than the in-line style, since the unit cells are arranged compactly in a square or rectangular shape; this allows the heat to be distributed more evenly and hence result in less cross-heating of the devices.

The zipper style was chosen for the power transistor cell layout used in this work, since only one gate orientation was allowed in this process, and even distribution of heat across the transistor cells was considered to be critical for not exacerbating the self-heating effect of the SOI devices. $256 \mu \mathrm{m}$ power transistor cell (which was found to be the periphery required to meet the output power requirement of the 3-stack PA designed in the next chapter) was realized by wiring 16 unit cells together as shown in Figure 3.9. C1 layer was used for interconnecting the unit cells, and the upper metal layers (UA to OB) were used for realizing the global nodes, since they have high current handling capability and low sheet resistance. The global gate and drain nodes were kept far apart to minimize the feedback capacitance $C_{g d}$, thereby reducing the 
likelihood of oscillation. Throughout the wiring process, multiple vias were used in order to reduce the equivalent via resistance and to be safe from electromigration; they were also used as a precaution against fabrication imperfection.

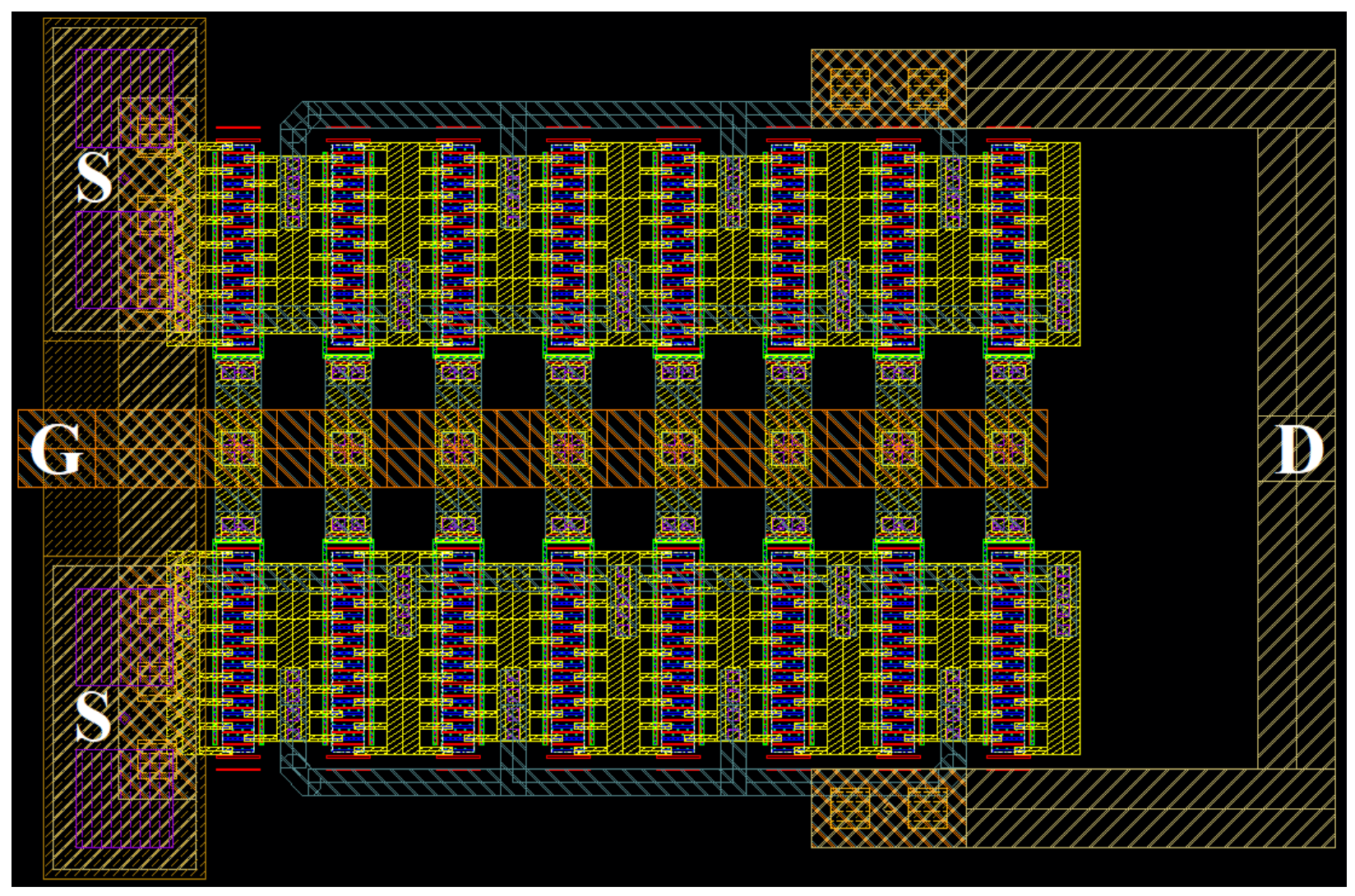

Figure 3.9: $256 \mu \mathrm{m}$ power transistor cell layout in "zipper" style.

As was done for the unit cell, PEX simulation was run on the power transistor cell layout, and the resulting PEX power transistor cell instance was used in the $f_{\max }$ simulation. Figure 3.10 shows $f_{\max }$ as a function of drain current density for the $256 \mu \mathrm{m}$ power transistor cell. It can be seen that while the current density where peak $f_{\max }$ occurs remained unchanged from that of the unit cell $(0.35 \sim 0.4 \mathrm{~mA} / \mu \mathrm{m})$, the peak $f_{\max }$ of the power transistor was reduced by $29.5 \%(395.8$ $\mathrm{GHz}$ to $279 \mathrm{GHz}$ ) due to the substantial increase in the layout parasitics. Table 3.2 shows the computed small-signal equivalent circuit parameters of the implemented $256 \mu \mathrm{m}$ power transistor cell. It can be seen that while the gate resistance decreased by $90 \%$ from that of the 
unit cell, the gate-drain capacitance increased by $1600 \%$, accounting for the significant reduction of $f_{\max }$.

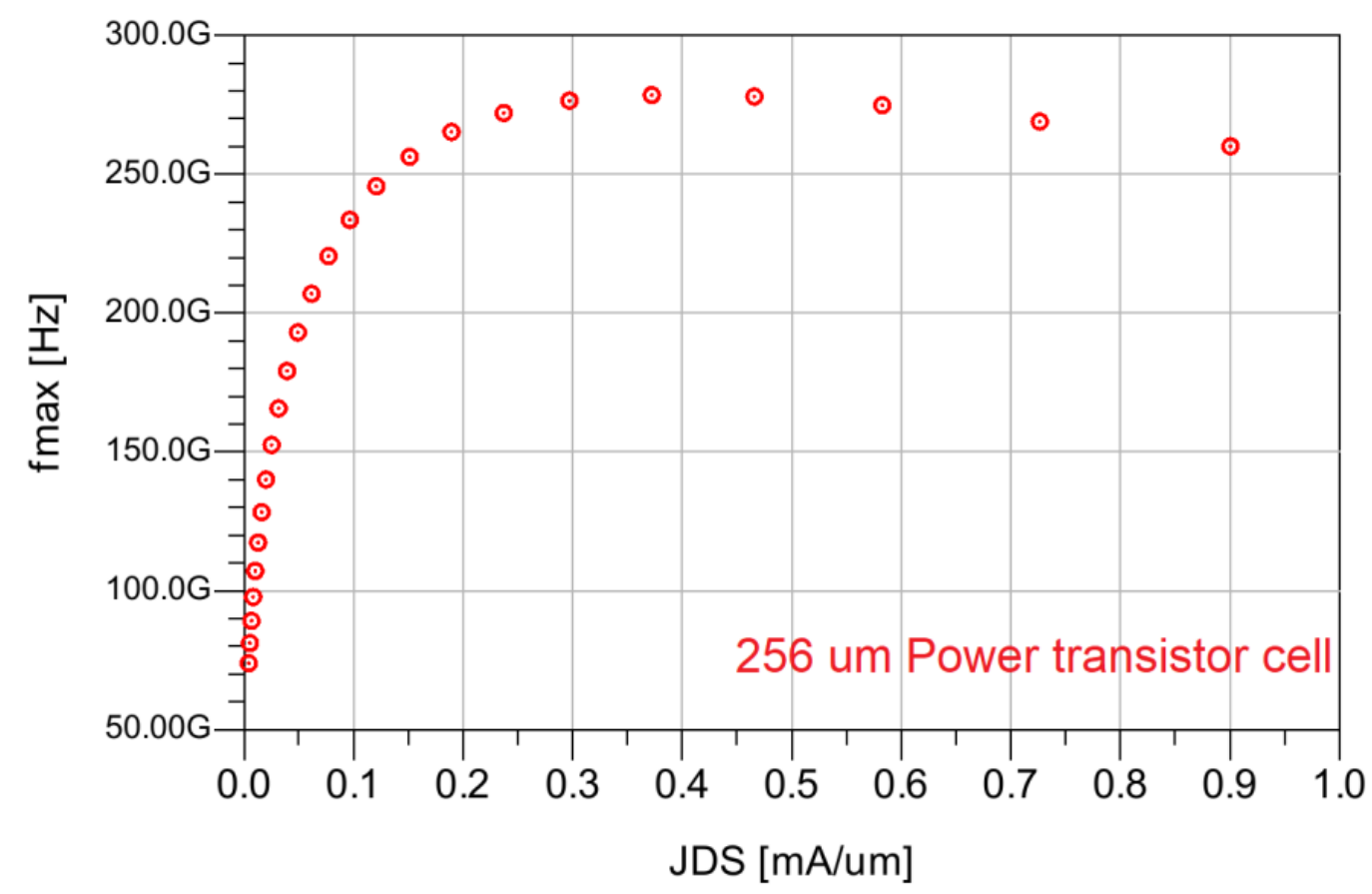

Figure 3.10: Extrinsic $f_{\max }$ versus drain current density for $256 \mu \mathrm{m}$ power transistor cell.

Table 3.2: Computed small-signal equivalent circuit parameters (at $28 \mathrm{GHz}$ ) of the $256 \mu \mathrm{m}$ power transistor cell

\begin{tabular}{|c|c|}
\hline & $\begin{array}{c}\text { Power transistor cell } \\
\mathbf{2 5 6} \boldsymbol{\mu \mathbf { m }}\end{array}$ \\
\hline $\boldsymbol{g}_{\boldsymbol{m}}[\mathbf{m S}]$ & 331.0 \\
\hline $\boldsymbol{C}_{g s}[\mathbf{f F}]$ & 163.0 \\
\hline $\boldsymbol{C}_{\boldsymbol{d}}[\mathbf{f F}]$ & 54.1 \\
\hline $\boldsymbol{C}_{g d}[\mathbf{f F}]$ & 84.6 \\
\hline $\boldsymbol{r}_{\boldsymbol{d s}}[\boldsymbol{\Omega}]$ & 16.0 \\
\hline $\boldsymbol{r}_{\boldsymbol{g}}[\boldsymbol{\Omega}]$ & 0.9 \\
\hline
\end{tabular}

\subsection{Summary}

In this chapter, the key RF/mmWave-optimized features of RF-SOI and PD-SOI devices were studied followed by the design and implementation of $16 \mu \mathrm{m}$ unit cell and $256 \mu \mathrm{m}$ power 
transistor cell in 45-nm RF-SOI process. The $f_{\max }$ of a unit cell was optimized by using unit gate width of $1 \mu \mathrm{m}$, double gate contacts, relaxed poly pitch, stacked-metal gate, and stair-case drain/source routing. For the $256 \mu \mathrm{m}$ power transistor cell layout, "zipper" style was used in order to have a compact layout and better heat distribution across the transistor cell. The peak extrinsic $f_{\max }$ of the $16 \mu \mathrm{m}$ unit cell and $256 \mu \mathrm{m}$ power transistor cell were found to be 395.8 $\mathrm{GHz}$ and $279 \mathrm{GHz}$, respectively. 


\section{Chapter 4}

\section{GHz 3-Stack Power Amplifier in 45-nm RF- SOI}

Transistor stacking is an effective way to achieve higher RF output power while overcoming the high knee voltage and low breakdown voltage limitations (i.e., gate-oxide and drain-source breakdown voltages) of advanced node RF-SOI devices. In 45-nm RF-SOI process, the floatingbody PD-SOI devices facilitate transistor stacking as they do not suffer from the body effect and have significantly low $\mathrm{S} / \mathrm{D}$ to the substrate parasitic capacitances (which translates to low parasitic capacitances at the intermediate nodes of the stack).Transistor stacking, being a practical alternative to the passive power combining, is more area-efficient than the passive power combining as the use of large on-chip output combiner can be eliminated. Furthermore, the higher input and output impedance of the stacked transistor cell make low-loss and wideband matching possible.

This chapter will begin with an overview of the transistor stacking technique, where its principle of operation will be studied. Then, the design and implementation of a Ka-band 3-stack PA will be presented with an emphasis on the layout-driven design method. This chapter will 
then conclude with EM-cosimulation results of the implemented PA chip and the measurement setup.

\subsection{Transistor Stacking Overview}

Transistor stacking refers to a series interconnection of transistors where common-gate-like amplifiers are stacked on top of a common-source amplifier as shown in Figure 4.1.

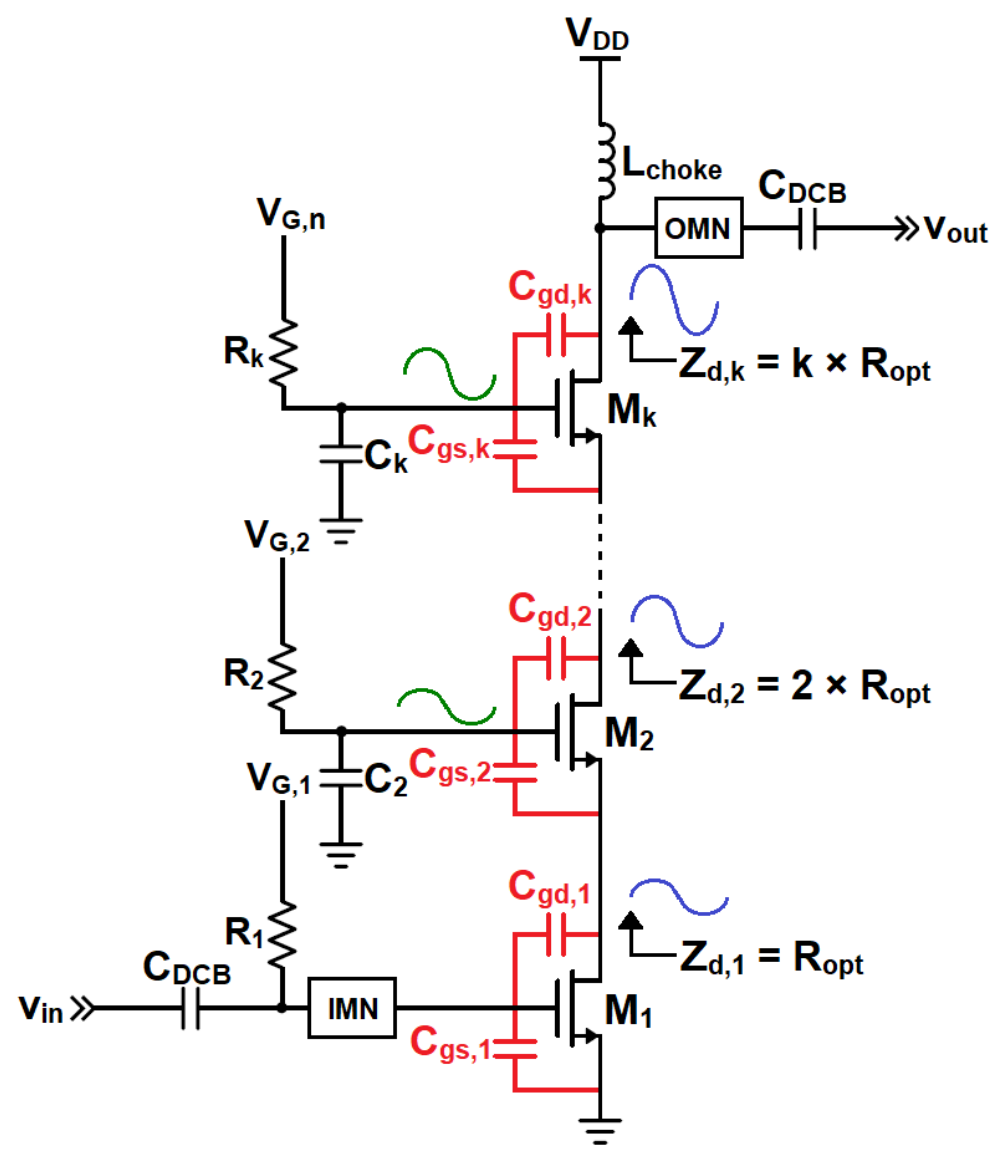

Figure 4.1: Basic configuration of an $N$-stack PA.

The stack topology enhances the voltage handling capability of the circuit and hence allows higher power supply voltage $V_{D D}$ to be used for the PA. For an $N$-stack topology comprised of $N$ identical transistors, $V_{D D}$ can be scaled to $N \times V_{D D \text {, max }}$ where $V_{D D \text {, max }}$ is the maximum power supply voltage allowed for a single transistor. However, the scaling of $V_{D D}$ is on the condition 
that the gate bias voltages $V_{G, n}(n=1,2, \ldots, N)$ are set correctly such that $V_{D D}$ is distributed equally among the individual transistors in the stack (i.e., $V_{D S, 1}=V_{D S, 2}=\ldots=V_{D S, N}$ ). With the equal DC drain-source voltage $V_{D S, n}$ across each transistor, the RF drain-source voltages of the individual transistors $v_{d s, n}$, must also be tuned to have equal magnitude and phase by loading the gates of the upper-stack transistors with the proper values of capacitance. Ultimately, the equal distribution of both DC and RF voltages allows the individual transistors to operate within the drain-source breakdown voltage (BVDSS) limit of a single transistor under a large-signal condition.

In the ideal lossless case, the in-phase combining of maximum $v_{d s, n, \max }$ (i.e., $v_{d s, 1, \max }+$ $\left.v_{d s, 2, \max }+\ldots+v_{d s, N, \max }\right)$ gives rise to $N \times\left(B V D S S-V_{k n e e}\right)$ voltage swing at the drain node of the top transistor (whose peak value can reach $N \times B V D S S$ in principle). If the RF drain currents of the individual transistors are constant, and they are in-phase, the theoretical maximum RF output power of an $N$-stack PA can be expressed as shown in (4.1) [41].

$$
P_{R F_{-} \text {out }}=\frac{N \cdot\left(B V D S S-V_{\text {knee }}\right) \cdot\left(I_{\max }-I_{\min }\right)}{8}
$$

It is evident from (4.1) that, in principle, by stacking $N$ transistors, the RF output power can be increased by a factor of $N$ from that of a single transistor. However, in reality, the output power is lower than the theoretical maximum value due to the losses in the transistors and non-ideal or out-of-phase drain-source voltage combining [40].

The maximum number of transistors that can be stacked is limited by the gate-oxide breakdown voltage of the transistors, the stability of the circuit, and the value of the top external gate capacitor [41]. It has been demonstrated, however, that practically no meaningful improvement in RF output power can be achieved beyond 3-stack [40]. As shown in Figure 4.2, if $0.5 \mathrm{~dB}$ loss per transistor is assumed, less than $0.5 \mathrm{~dB}$ improvement in RF output power can be 
achieved with the addition of $4^{\text {th }}$ transistor. It is also important to note that while higher stack results in a higher RF output power, corresponding PAE is lower due to the higher resistive losses in the transistors.

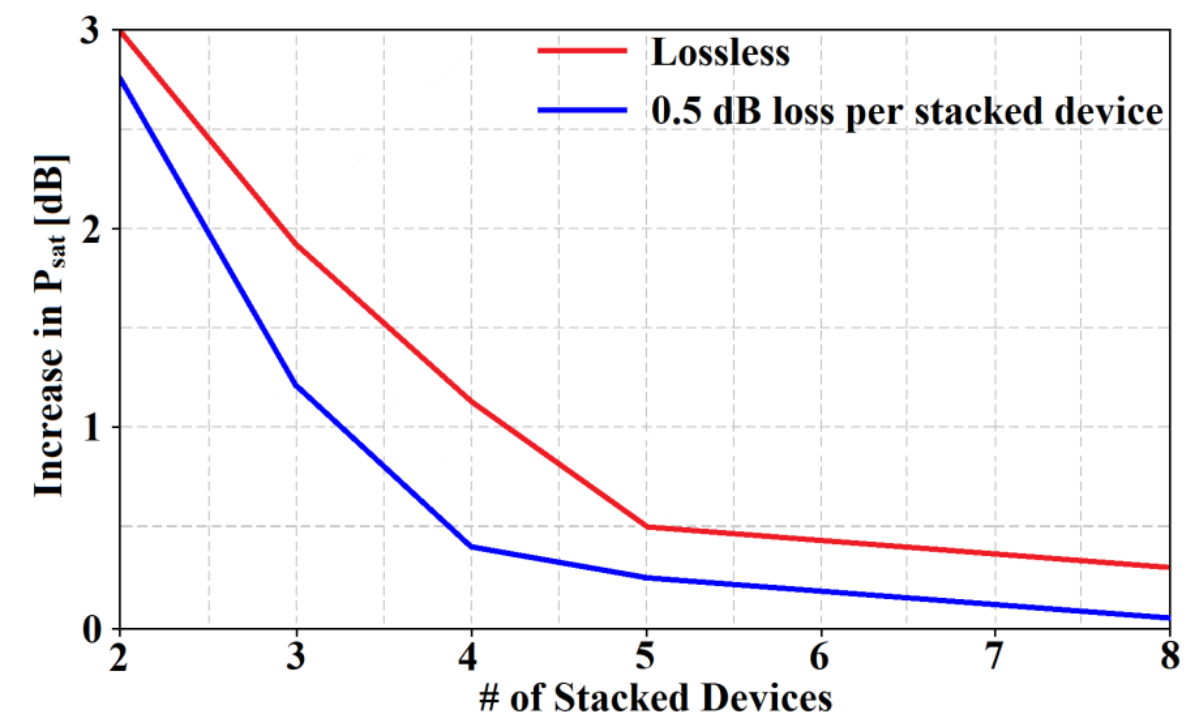

Figure 4.2: Achievable increase in the saturated output power from a common-source PA versus number of stacked devices [40].

\subsubsection{Determination of Gate Capacitance}

Stack topology differs from the cascode topology in that the gates of the upper-stack transistors are not grounded at the frequency of operation but loaded with finite external capacitors $C_{k}(k=2$, $3, \ldots, N)$. The finite impedance at the gate gives rise to finite RF voltage $v_{g, k}$ on the gates of the upper-stack transistors whose magnitude is determined by the voltage division across $C_{k}$, and the parasitic gate-source capacitance $C_{g s, k}$. It is important to note that in order for the gate voltage swing to increase correspondingly with the increase in the drain voltage swing as one moves up the stack, the values of $C_{k}$ need to decrease as one moves up the stack; this ensures the peak value of the instantaneous gate-drain voltage $v_{G D, k}$ is kept below the gate-drain breakdown voltage limit of a single transistor (assuming the RF gate and drain voltages are in-phase). 
In addition to controlling the RF gate voltages, $C_{k}$ also determines the impedance seen from the drain of $(k-1)^{\text {th }}$ transistor in the stack. Under the simplifying assumption that the smallsignal output resistance $r_{o}$ is infinite, and the parasitic drain-source capacitance $C_{d s}$ is zero, the impedance seen from the drain of $(k-1)^{\text {th }}$ transistor can be expressed as shown in (4.2) [40], where $Z_{d, k}$ is the impedance seen from the drain of $k^{\text {th }}$ transistor.

$$
Z_{d, k-1}=\frac{C_{g s, k}+C_{k}+C_{g d, k}\left(1+g_{m, k} Z_{d, k}\right)}{\left(g_{m, k}+s C_{g s, k}\right)\left(C_{g d, k}+C_{k}\right)}, k=2,3, \ldots, N
$$

In order to have maximum drain-source voltage swings across the individual transistors in the stack, the output nodes of the upper-stack transistors must be presented with scaled multiples of $R_{\text {opt }}$ (i.e., $2 R_{\text {opt }}, 3 R_{\text {opt }}$ ), where $R_{\text {opt }}$ is the real part of the optimum fundamental load impedance of a single transistor. Consequently, by setting $Z_{d, k-I}$ to $(k-1) \times R_{\text {opt }}$ and $Z_{d, k}$ to $k \times R_{\text {opt }}$ in (4.2) (which is a valid assumption for operating frequency $f_{o}$ much lower than $f_{T}$ ), and performing mathematical manipulation on (4.2), $C_{k}$ can be expressed analytically as shown in (4.3) [40].

$$
C_{k}=\frac{C_{g s, k}+C_{g d, k}\left(1+g_{m, k} R_{o p t}\right)}{(k-1) g_{m, k} R_{o p t}-1}, k=2,3, \ldots, N
$$

In summary, the proper selection of $V_{G, n}$, and $C_{k}$ values, and the linear scaling of $R_{o p t}$ allow the stack PA to achieve higher RF output power while ensuring the reliability of each transistors in the stack. The scaling of $R_{\text {opt }}$ is also advantageous in terms of output matching network design, since higher $R_{\text {opt }}$ reduces the impedance transformation ratio up to $50 \Omega$, hence simplifies the matching complexity.

\subsubsection{Intermediate Node Matching}

If $f_{o}$ is a significant fraction of $f_{T}$, there may be phase misalignments between the RF drain-source voltages of the individual transistors due to the substantial amount of inter-nodal parasitic 
capacitances that arise from the transistor cell layout and metal interconnections to the conductive silicon substrate. The inter-nodal parasitic capacitances, whose effect becomes more acute in proportion to the increase in frequency, cause $Z_{d, k}$ to have capacitive reactance, thereby negating the purely resistive approximation, which was valid for $f_{o}$ much lower than $f_{T}$. In addition to the phase misalignments of the RF drain-source voltages, the inter-nodal parasitic capacitances cause the RF current to leak through them (primarily through $C_{g s, k}$ ), which, in turn, reduces the amount of RF current delivered to the load. Consequently, the out-of-phase combining of the RF drain-source voltages and the reduced RF current delivered to the load results in lower RF output power, PAE, and small-signal gain of a PA.

The performance of an $N$-stack PA, which operates at a significant fraction of the transit frequency, can be optimized by correcting the phase misalignments in the RF drain-source voltages, so that they combine in-phase to give the highest voltage swing at the drain node of the top transistor. To this end, a matching element can be introduced at each intermediate node of the stack to resonate out the capacitive reactance present at that node. Figure 4.3 shows the three different intermediate node matching techniques that have been reported, namely, shunt capacitor, series inductor, and shunt inductor [40]. Since intermediate node matching was not used in the $N$-stack PA presented in this work, no further discussion will be made of these matching techniques. However, more details on these intermediate matching techniques and the analytical expressions for computing the matching component values can be found in [40]. The use of intermediate node matching has made it feasible to use the transistor stacking technique effectively even at every high mmWave frequencies. 


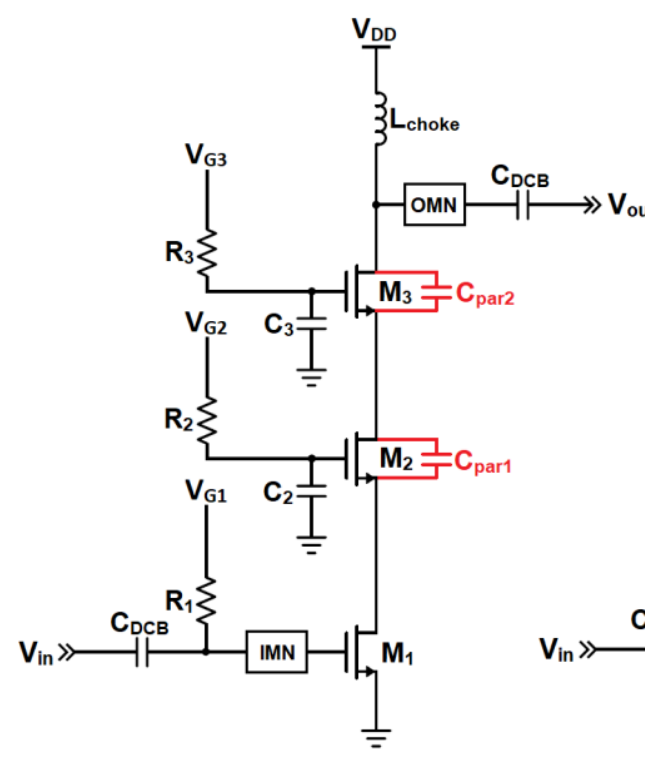

(a)

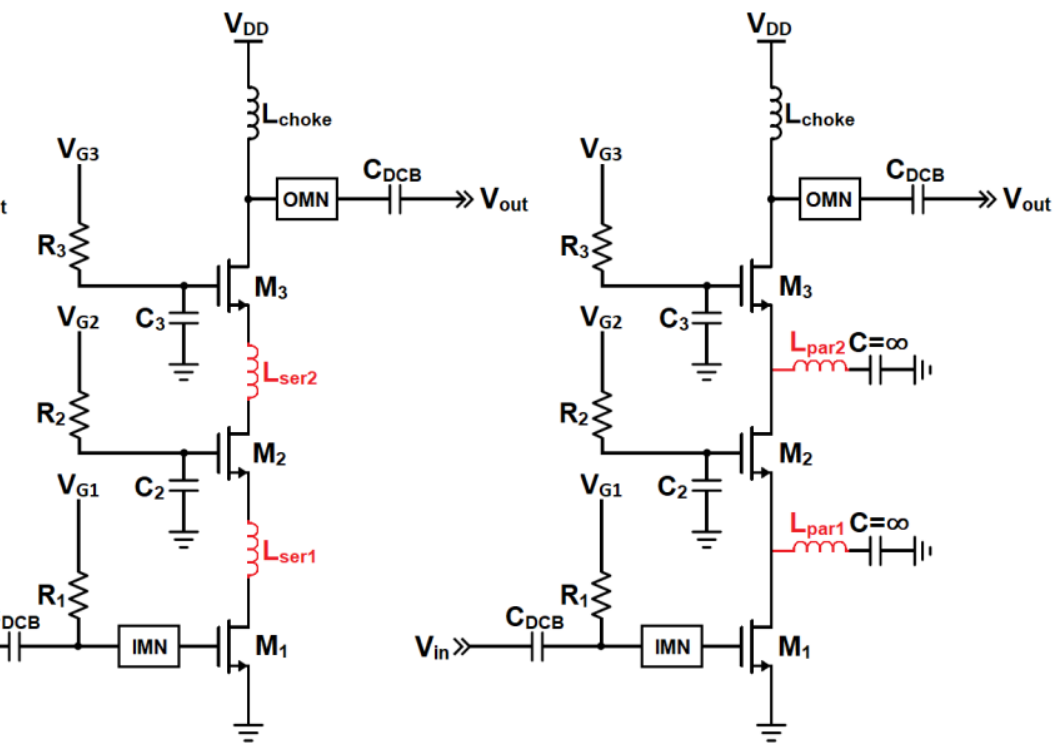

(b)

(c)

Figure 4.3: Intermediate node matching techniques (a) shunt capacitor (b) series inductor (c) shunt inductor.

\subsection{3-Stack PA Design in 45-nm RF-SOI}

The impact layout parasitics have on the performance of a PA becomes more pronounced in proportion to the increase in frequency and power; hence, overlooking their effects may result in unwanted performance degradation, frequency shift, and/or instability. Nevertheless, as the occurrence of these parasitics is inevitable, it is critical to capture their effects thoroughly by exploiting parasitic-extraction (PEX) and electromagnetic (EM) simulations, and incorporate them into the design.

In this section, the design and implementation of a Ka-band 3-stack PA will be presented with an emphasis on the layout-driven design method. In order to achieve the target output power of $20 \mathrm{dBm}$, the PA employs three instances of the $256 \mu \mathrm{m}$ power transistor cell that is implemented in Section 3.2.4 (which was sized for $20.5 \mathrm{dBm}$ saturated output power, leaving 0.5 $\mathrm{dB}$ margin for the losses in the output matching network). The PA operates from a 3.3V power 
supply, and the gates of the bottom, middle, and top transistors were biased at $0.55 \mathrm{~V}, 1.65 \mathrm{~V}$, and $2.75 \mathrm{~V}$, respectively. The gate bias voltages were chosen, such that each transistor in the stack operates at the optimum current density for maximum power gain, and each transistor has $V_{D S}$ of $1.1 \mathrm{~V}$ (i.e., $3.3 \mathrm{~V}$ power supply voltage distributed equally among the three transistors).

\subsubsection{Layout-driven Design and Implementation}

The layout-driven design method makes use of the SnP (n-port S-parameter) file of the physical layout that gets generated from EM simulations (which uses Integrand's EMX tool embedded within the Cadence Virtuoso environment). By doing so, the parasitics and distributed effects associated with the layout are fully taken into account in the design. In order to generate the $\mathrm{SnP}$ file of the die layout, the active and passive components other than the on-chip inductors and interconnects were removed from the layout; then, edge ports were placed at every component connection points. The edge ports create pins on the schematic instance of the SnP file so that the PEX transistor instances and the S2P instances of the capacitor layout can be wired to their corresponding pins in the EM-cosimulation test bench as shown in Figure 4.4.

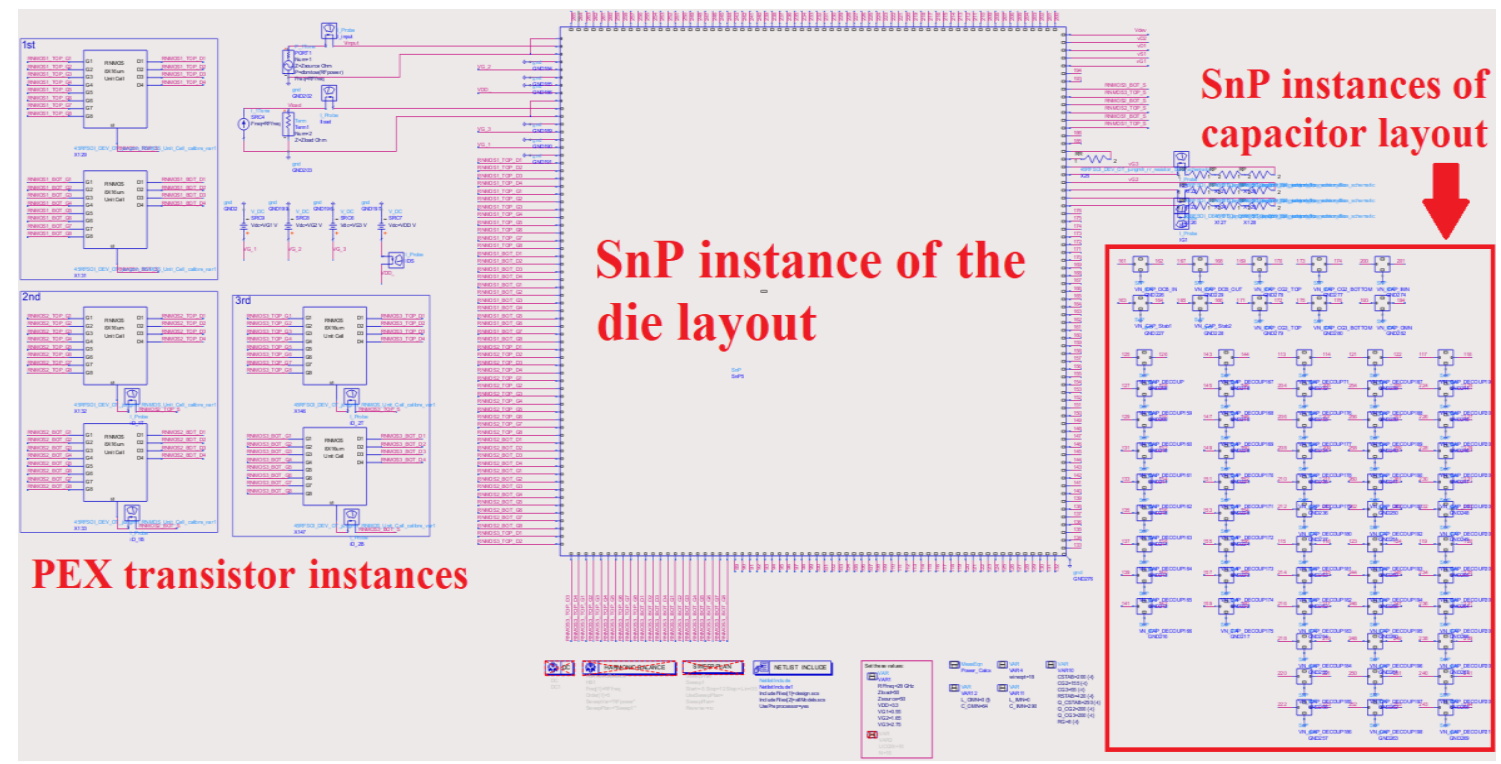

Figure 4.4: EM-cosimulation test bench of the 3-stack PA. 
The first step in the design was the floor planning of the die; the die area was determined by placing Ground-Signal-Ground (G-S-G) RF pads at the east and west of the die, and PPPPP type DC pads at the north and south of the die. Since $150 \mu \mathrm{m}$ pitch was required for both RF and DC pads, the minimum size of the die was more or less fixed. The floor plan of the die and the function of each pad are shown in Figure 4.5. It should be noted that edge ports were placed on all of the pads in order to capture the effects of ground inductance and the loading effects of the RF and DC pads. The edge ports were also used to create pins on the schematic instance of the die layout SnP file for source, load, and ground connections.

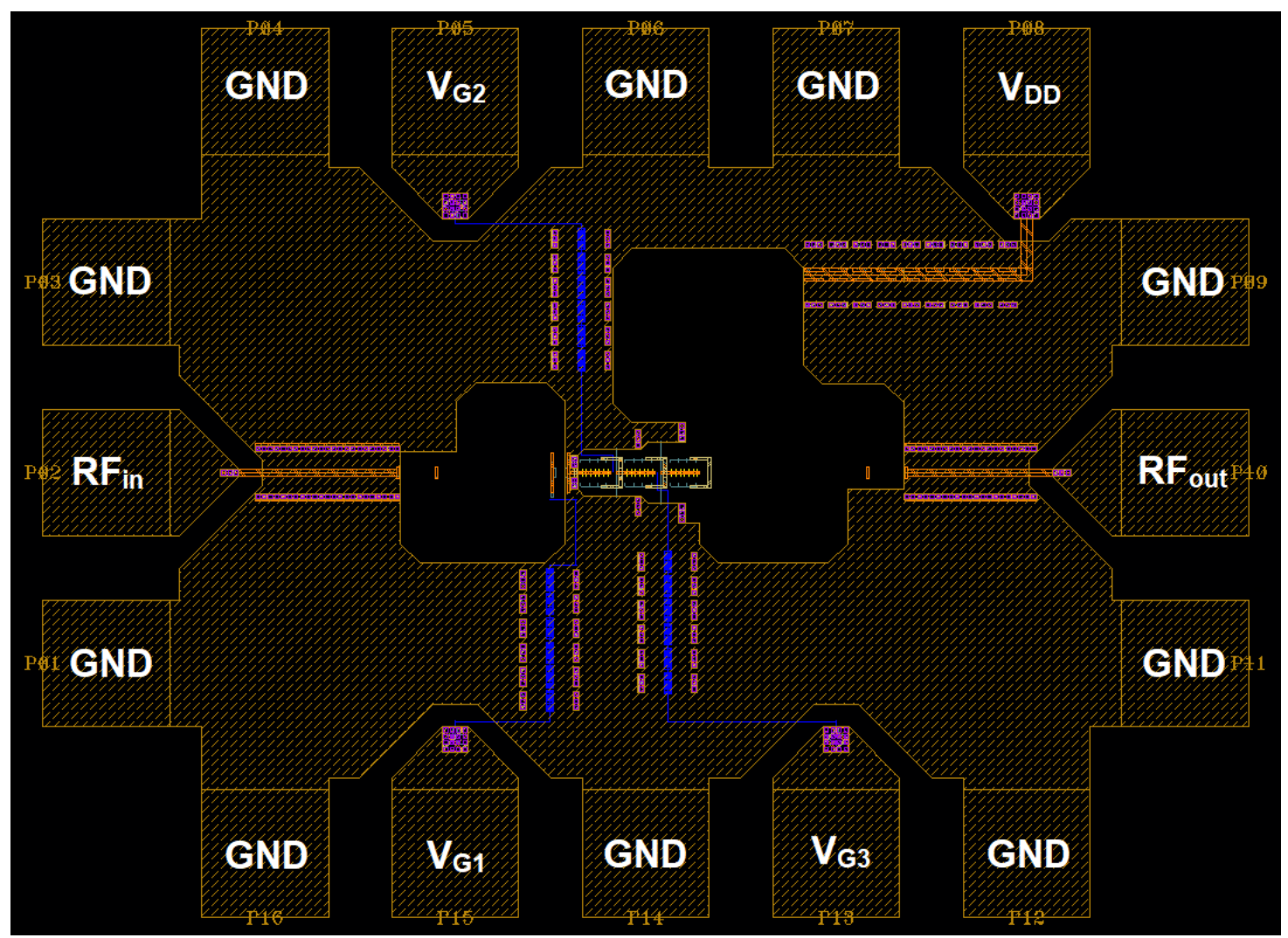

Figure 4.5: Floor plan of the PA chip with bias lines and no input and output matching networks.

In order to provide low impedance return paths for the RF currents, a global ground plane was realized on LD layer, which is thick aluminum, and the highest layer in the BEOL metal stack. As shown in Figure 4.6, the top surface of the die was flooded with LD layer except for 
areas where there are/will be on-chip inductors and transistor cells. The low impedance global ground plane on the top layer prevents fields from entering the substrate and ensures the ground connections of all the active and passive components are at the same reference potential. Furthermore, it reduces the gain degradation of the CS amplifier (bottom transistor), which occurs due to source degeneration.

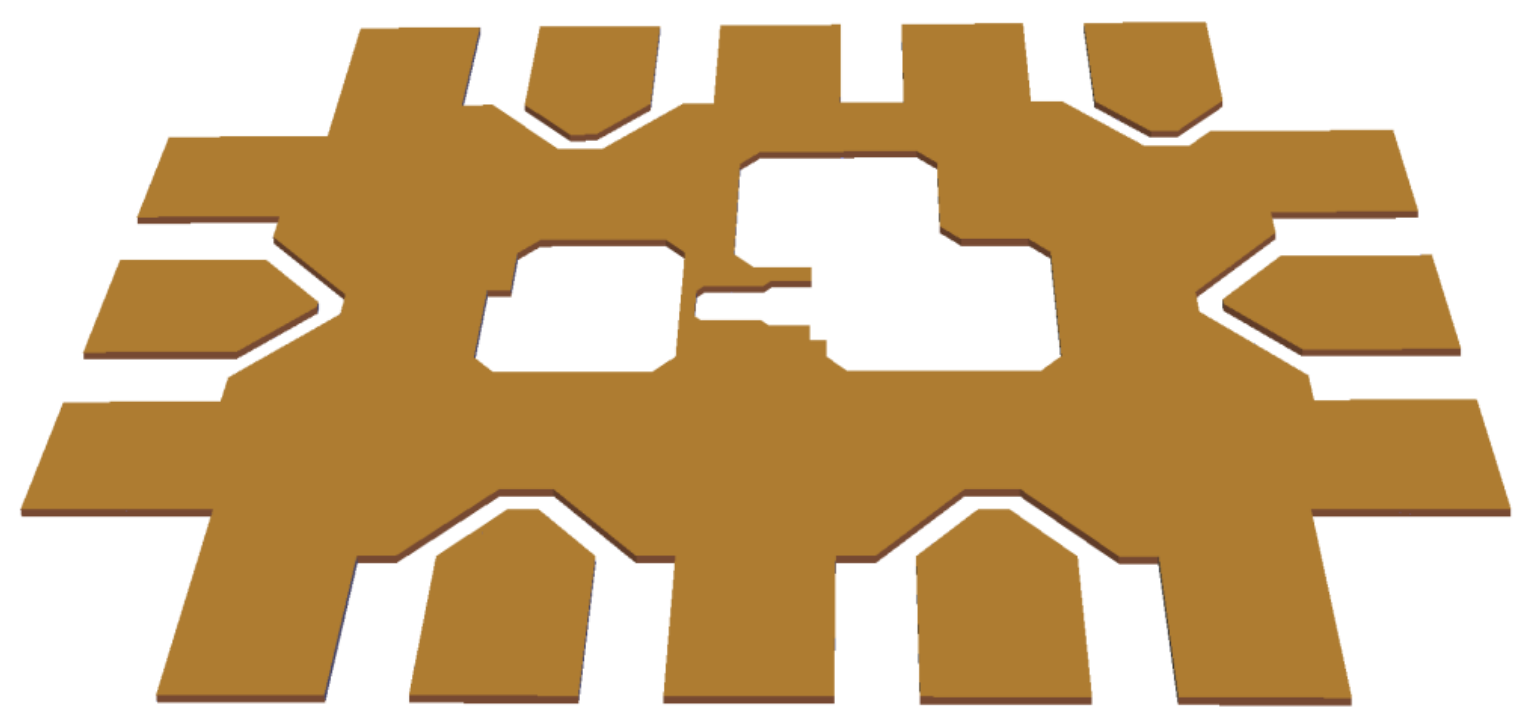

Figure 4.6: Global ground plane on LD layer.

The 3-stack topology was realized by placing three identical $256 \mu \mathrm{m}$ power transistors cells in the center of the die and interconnecting them with traces on OB layer. OB layer, which is thick copper, was chosen to minimize power loss and to provide sufficient current handling capability. It should be noted that the lengths of these interconnects were kept short to minimize the parasitic inductances. With the interconnects in place, the transistor cores and wiring up to C1 layer were removed, and only UA up to OB layers were retained in the transistor cell layout; then, as shown in Figure 4.7, edge ports were placed to create pins so that the PEX transistor instances (which contains the parasitic resistances and capacitances extracted from the polysilicon layer up to $\mathrm{C} 1$ layer in the transistor cell layout) can be used in the EM-cosimulation of the PA. 


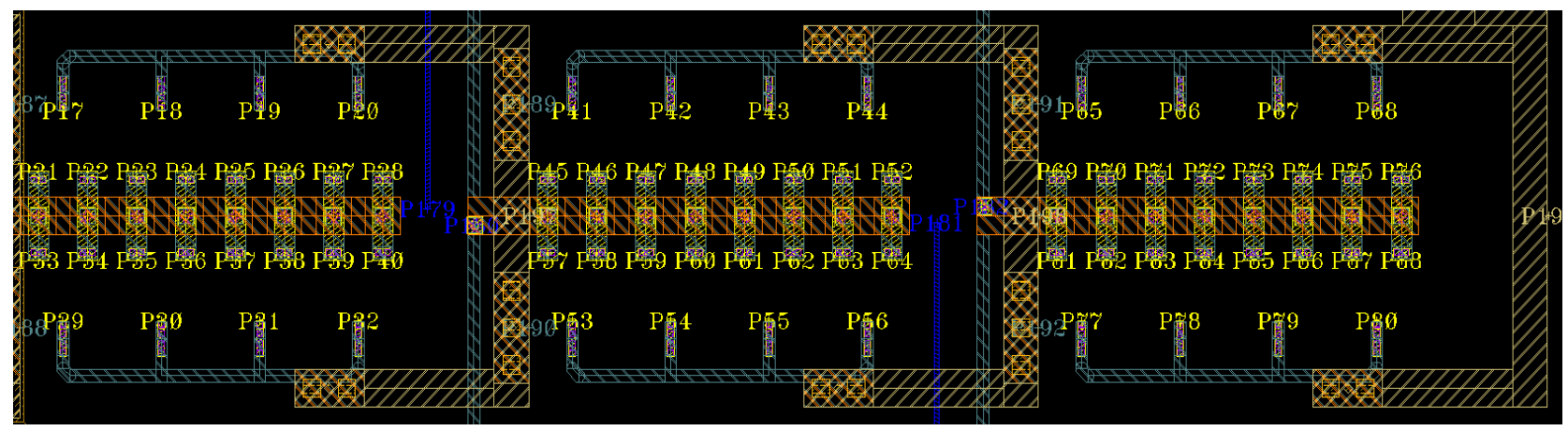

Figure 4.7: Placement of edge ports to capture the effects of the interconnect and via inductances, as well as the coupling between the interconnects (OB down to UA).

Since a large quiescent current flows in the drain bias line, it is critical to ensure that the bias line has high current handling capability to be safe from electromigration, and has low resistance to minimize the power supply voltage drop along the line. To this end, the drain bias line was implemented on OA layer (which is thick copper) with a line width of $10 \mu \mathrm{m}$. On the contrary, since negligible currents flow in the gate bias lines, the gate bias lines were implemented on M1 layer with a line width of $0.3 \mu \mathrm{m}$. Finally, the RF signal lines were implemented on OA layer with a line width of $5 \mu \mathrm{m}$ to have low insertion loss. Throughout the design, multiple or arrays of vias were used in order to reduce the equivalent via resistance and to be safe from electromigration. Furthermore, they were used as a precaution against fabrication imperfection, which can lead to non-functioning or failure-prone circuits. For supply decoupling, instead of using a single large-value capacitor, many $420 \mathrm{fF}$ decoupling capacitors were placed along the bias lines to realize a large capacitance $(5 \sim 7.5 \mathrm{pF})$; this approach was taken, since large-value capacitors have low self-resonant frequency (SRF). The AC reference point of the decoupling capacitors was set by connecting the ground terminal of the decoupling capacitors to the low impedance ground plane on LD layer using stacked vias. For the input and output DC block capacitors, $2 \mathrm{pF}$ was used in order to have a small series impedance at the frequency of operation. 
Table 4.1 shows the initially computed and final optimized values of the external gate capacitors. The initial values of the external gate capacitors were computed using (4.3) where $g_{m}$, $C_{g s}, C_{g d}$ values of the power transistor cell were obtained from Table 3.2. The $R_{\text {opt }}$ of a single power transistor was found using a load line analysis and $10.5 \Omega$ was used in the external gate capacitor computation. Based on the transient response of the PA, the initial external gate capacitor values were fine-tuned later using a CAD simulator such that each transistor in the stack has optimum $v_{d s}$ at the maximum input drive level.

Table 4.1: Computed and optimized values for the external gate capacitor.

\begin{tabular}{|c|c|c|}
\hline $\boldsymbol{k}$ & $\begin{array}{c}\text { Computed } \boldsymbol{C}_{\boldsymbol{k}} \\
{[\mathbf{f F}]}\end{array}$ & $\begin{array}{c}\text { Optimized } \boldsymbol{C}_{\boldsymbol{k}} \\
{[\mathrm{fF}]}\end{array}$ \\
\hline 1 & - & - \\
\hline 2 & 220 & 310 \\
\hline 3 & 91 & 110 \\
\hline
\end{tabular}

In order to keep the design symmetric, the external gate capacitor per stack was realized using two identical capacitors of value $0.5 C_{k} \mathrm{fF}$ as shown in Figure 4.8. As in the case of the supply decoupling capacitors, the AC reference point of the external gate capacitors was set by connecting the ground terminals of the capacitors to the low impedance ground plane using stacked vias. Furthermore, as was done for the transistor cells, edge ports were placed at the connection points of the capacitors so that the S2P instances of the capacitor layout can be used in the EM-cosimulation of the PA. 


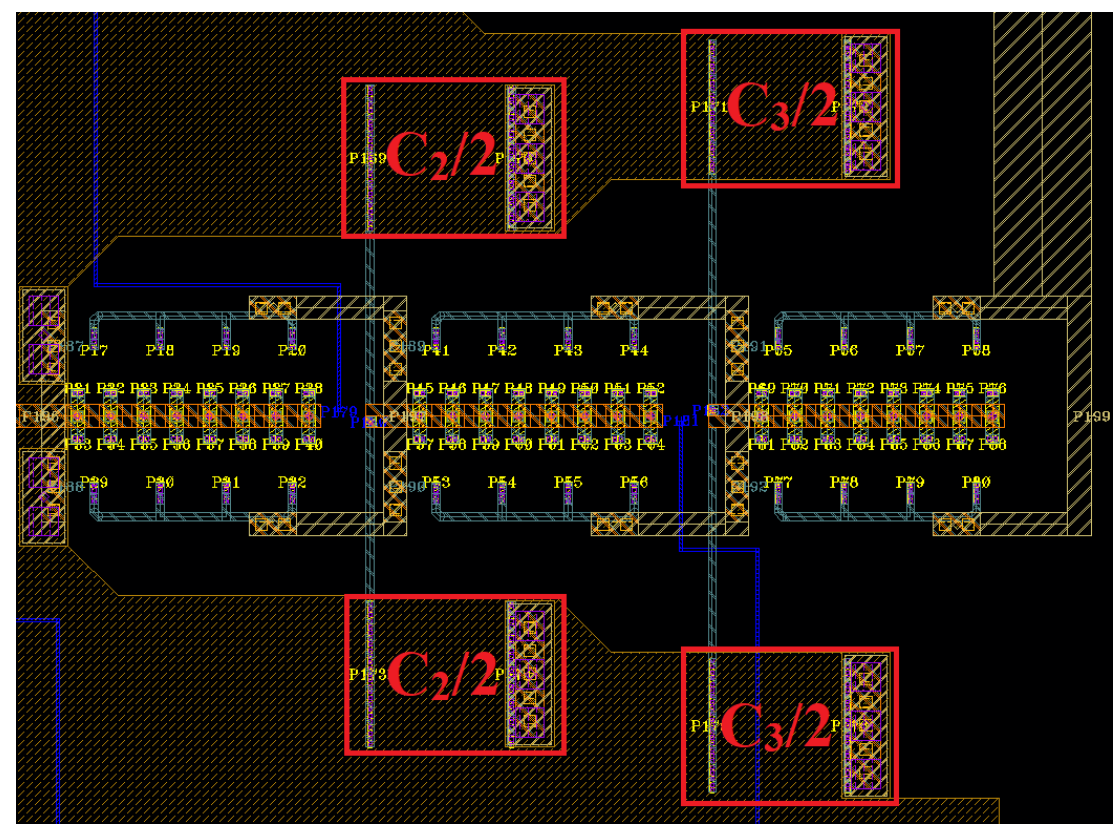

Figure 4.8: Realization of the external gate capacitor per stack using two identical capacitors of value $0.5 C_{k}$.

For all the capacitors in the design, VNCAP (Vertical Neutral Capacitor), which is a type of MOM (Metal-Oxide-Metal) capacitor was used; VNCAP makes use of the available BEOL metal layers and does not require additional process masks; hence, it is cheaper than the MIM (Metal-Insulator-Metal) capacitor, which requires additional process masks for the high-k dielectric layer. VNCAP was chosen over the MIM capacitor, not only because it offers higher $Q$ than the MIM capacitor for a given value of capacitance, but also because it offers layout convenience attributed to its perfectly symmetrical structure. Figure 4.9 shows a VNCAP made with interdigitated metal fingers on four consecutive layers. In order to achieve the highest $Q$ for a given value of capacitance, the length-to-width ratio of the capacitor layout was made close to 1. Furthermore, since the capacitor layout suffers from high frequency distributed effects, EM simulations were run on the capacitor layout to obtain the accurate dimensions that gives the desired capacitance value (while keeping the length-to-width ratio close to 1). The resulting S2P instances of the capacitor layout were used in the EM-cosimulation of the PA. 


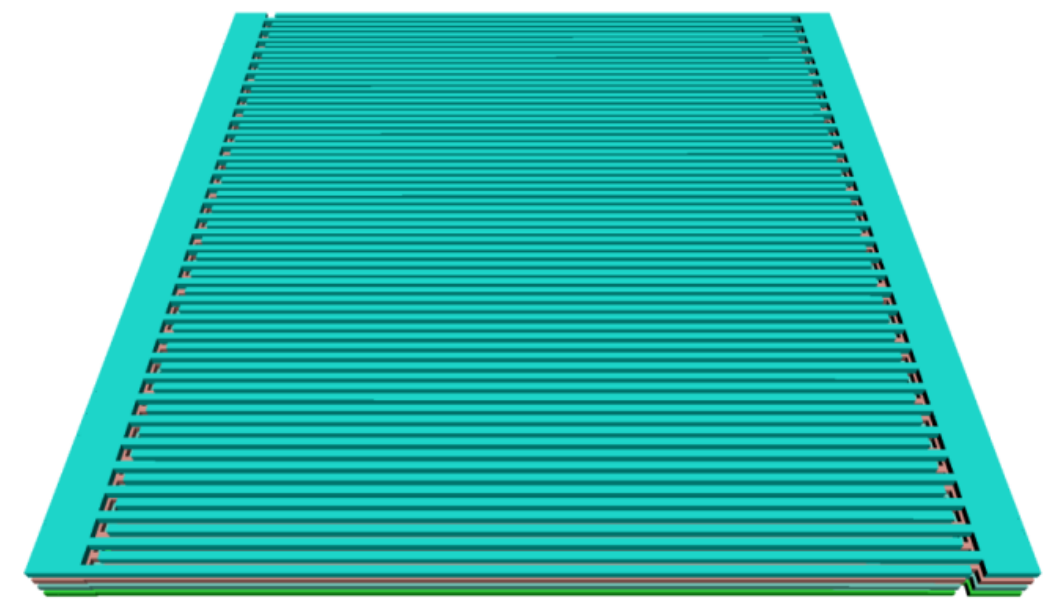

Figure 4.9: VNCAP made with four consecutive metal layers (M1 to C1).

Polysilicon resistors were used as gate bias resistors and an $R C$ stabilization element in the design. Polysilicon resistors are favored in $\mathrm{RF} / \mathrm{mmW}$ ave circuits as there is no distributed capacitance associated with it other than the shunt capacitance between the polysilicon and the substrate. In order to ensure the low-frequency stability of the PA (which requires suppressing the gain peaking at low frequency), a parallel $R C$ stabilization circuit was added in series with the gate of the CS amplifier. For the stabilization resistor and capacitor, $420 \Omega$ and $400 \mathrm{fF}$ were chosen as they were found to offer the best trade-off between stability and small-signal gain. With the $R C$ stabilization circuit in place, the PA was verified to be unconditionally stable from $\mathrm{DC}$ up to the $f_{\max }$ of the power transistor.

Lumped inductors were used for input and output matching, as well as for RF choke in the output bias network. Planar inductors with sufficiently high SRF were realizable on-chip, since the required inductance at $28 \mathrm{GHz}$ was small (up to a couple of hundreds of $\mathrm{pH}$ ). Nevertheless, since inductor structures occupy a significant portion of the die area compared to the transistor cells or other passive components, it was critical to have a compact inductor layout. To this end, a double-layer spiral inductor was realized by interconnecting the spirals on the two consecutive layers as shown in Figure 4.10. The double-layer spiral structure allows the 
realization of a given inductance in a compact area, since the mutual inductance of the two spirals adds to the inductance of the individual spiral [23].

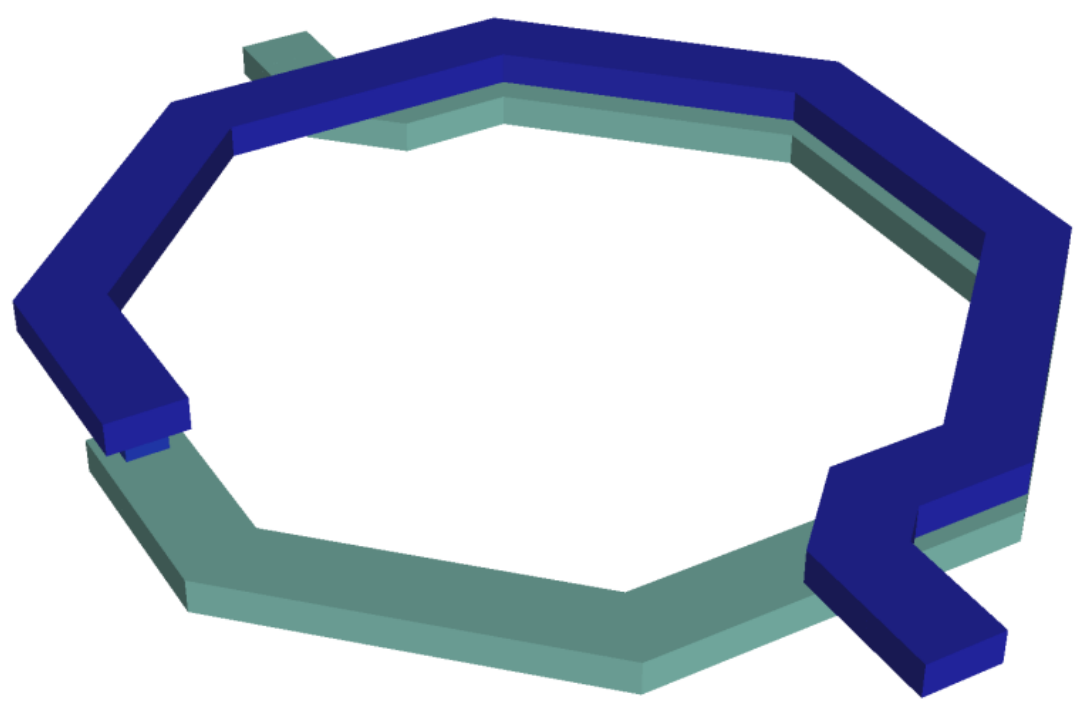

Figure 4.10: On-chip double-layer spiral inductor made with spirals on OA and OB layer.

The spirals were realized on OA, and OB layers, since these layers have low resistivity and are far away from the substrate (i.e., less losses due to substrate coupling). The patterned ground shield for preventing Eddy current was not needed, since 45-nm RF-SOI uses HR-substrate and the inductor structures were physically separated from the substrate by the BOX layer. The spirals were made in an octagonal shape to reduce the corner resistances, and the number of turns was kept to 1.5 to keep the inner area of the inductor hollow (thereby improving the $Q$ of the inductor). Furthermore, the line width and the area of the inductor were tuned to achieve high $Q$ at $28 \mathrm{GHz}$. Lastly, in order to reduce the capacitive coupling to the ground plane, which can cause a reduction in the inductance, peak $Q$, and SRF of the inductors, cutouts were made on the ground plane around the inductor structure. A distance of 5 to 6 line widths were kept between the edges of the inductor structure and the ground plane.

On-chip inductors suffer from high-frequency effects such as substrate coupling, interwinding/layer-to-layer capacitive coupling, skin effect, and proximity effect; hence, it is 
imperative to run EM simulations to obtain the accurate performance of the inductors. The EMsimulated performance of the on-chip inductors that are used in the design is summarized in Table 4.2. From the simulations of various sizes of double-layer spiral inductors, it was observed that for small-value inductors (i.e., up to a couple of hundreds of $\mathrm{pH}$ ), the typical $Q$ (at $28 \mathrm{GHz}$ ) and SRF that can be achieved were 20 30 and 60 140 GHz, respectively. On the other hand, for large-value inductors (i.e., close to $1 \mathrm{nH}$ ), the typical $Q$ and SRF that can be achieved were 15 20 and 30 40 GHz, respectively.

Table 4.2: Inductance, quality factor, and self-resonant frequency of the input and output matching and RF choke inductors.

\begin{tabular}{|c|c|c|c|}
\hline & $\mathbf{L}_{\text {IMN }}$ & L $_{\text {OMN }}$ & L RF Choke $_{\text {Ret }}$ \\
\hline Inductance [pH] (at 28 GHz) & 220 & 160 & 310 \\
\hline Q (at 28 GHz) & 25.5 & 29.5 & 23.3 \\
\hline SRF [GHz] & 103.7 & 132.7 & 57.8 \\
\hline
\end{tabular}

In order to design the input matching network of the PA, $50 \Omega$ signal source and load were connected to the RF input and output port of the PA, respectively, in the EM cosimulation test bench (as shown in Figure 4.4). Then, the impedances seen at the reference planes shown in Figure 4.11 were found with harmonic balance simulations. Finally, $Z_{\text {in_Gate }}$ was conjugately matched to $Z_{\text {in_RFin_PAD }}$ using a single-section low-pass L match. This matching scheme allowed the capacitive reactance of the input RF pad and the impedance of the RF signal lines to be effectively absorbed into the matching network. 


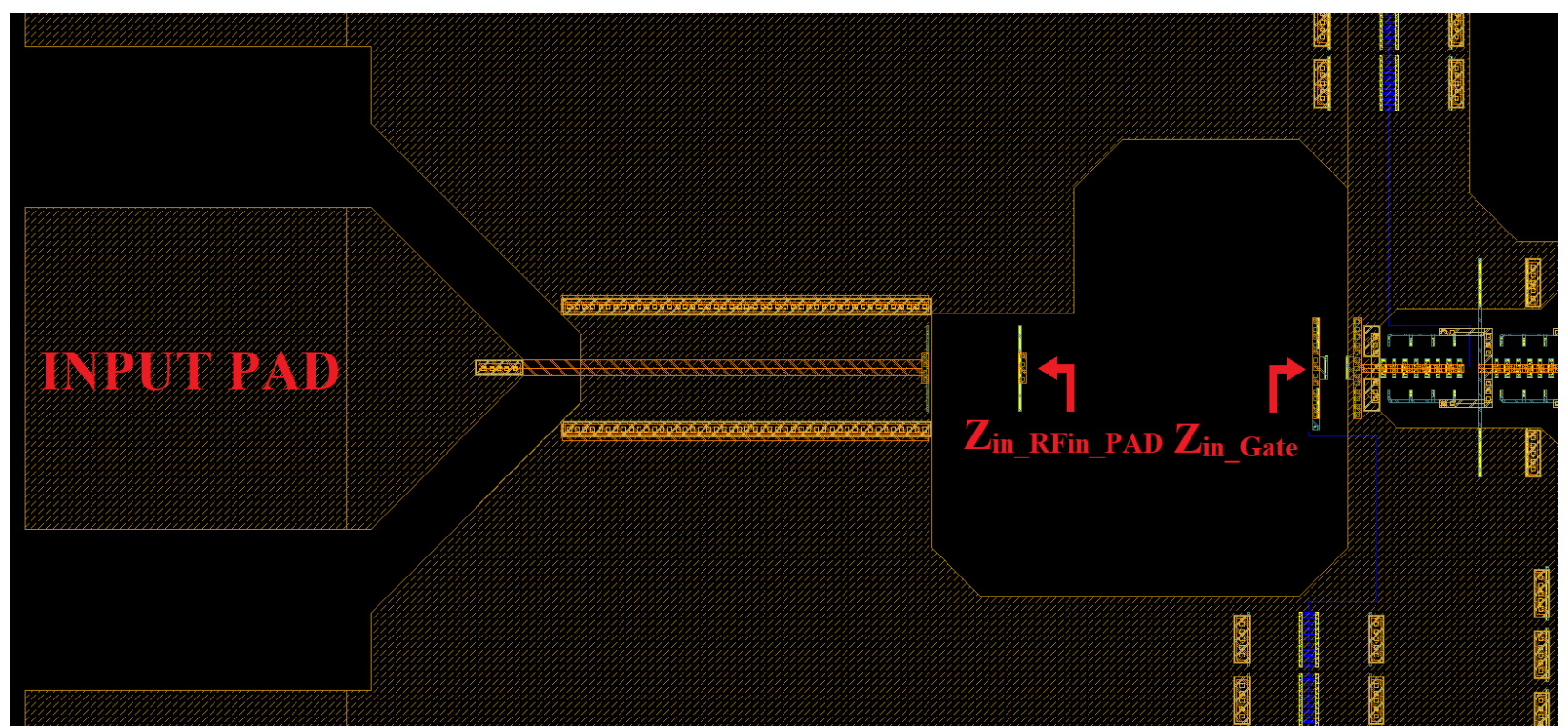

Figure 4.11: Impedance looking towards the RF input pad $\left(Z_{\text {in__RFin_PAD }}\right)$ and impedance looking into the input of the PA $\left(Z_{\text {in_Gate }}\right)$.

In order to design the output matching network of the PA, load-pull simulations were run with the input matching network in place. With a load tuner connected at the reference plane shown in Figure 4.12, the load impedance and the source power were swept to find the optimum load impedance that results in the highest PAE for $20 \mathrm{dBm}$ saturated output power. However, it was found that at $20 \mathrm{dBm}$ saturated output power, the RF drain-source voltages swing above the BVDSS limit of the device (2.2V for 40-nm PD-SOI devices [42]), and the gain compression is somewhat severe; hence, in order to ensure the reliability of the devices and to improve the linearity of the PA, the target output power was adjusted to $19.5 \mathrm{dBm}$. Figure 4.13 shows the PAE contours in $0.5 \%$ steps for $19.5 \mathrm{dBm}$ output power. It can be seen that the optimum load impedance is primarily resistive as a result of $f_{o}$ being much smaller than the $f_{T}$ of the power transistor $\left(f_{o}\right.$ is $28 \mathrm{GHz}$, and $f_{T}$ was found to be approximately $\left.260 \mathrm{GHz}\right)$. Furthermore, the real part of the optimum load impedance is approximately scaled multiples of $R_{\text {opt }}(\mathrm{i} . \mathrm{e} ., 3 \times 10.5 \Omega)$ as predicted by the theory. Although, $32.6+\mathrm{j} 8.7 \Omega$ was found to be the optimum load impedance 
that results in the highest PAE, the output of the stacked transistor cell was matched to $32.6+$ $\mathrm{j} 6.3 \Omega$ as it offered the best trade-off between PAE, output power, and linearity.

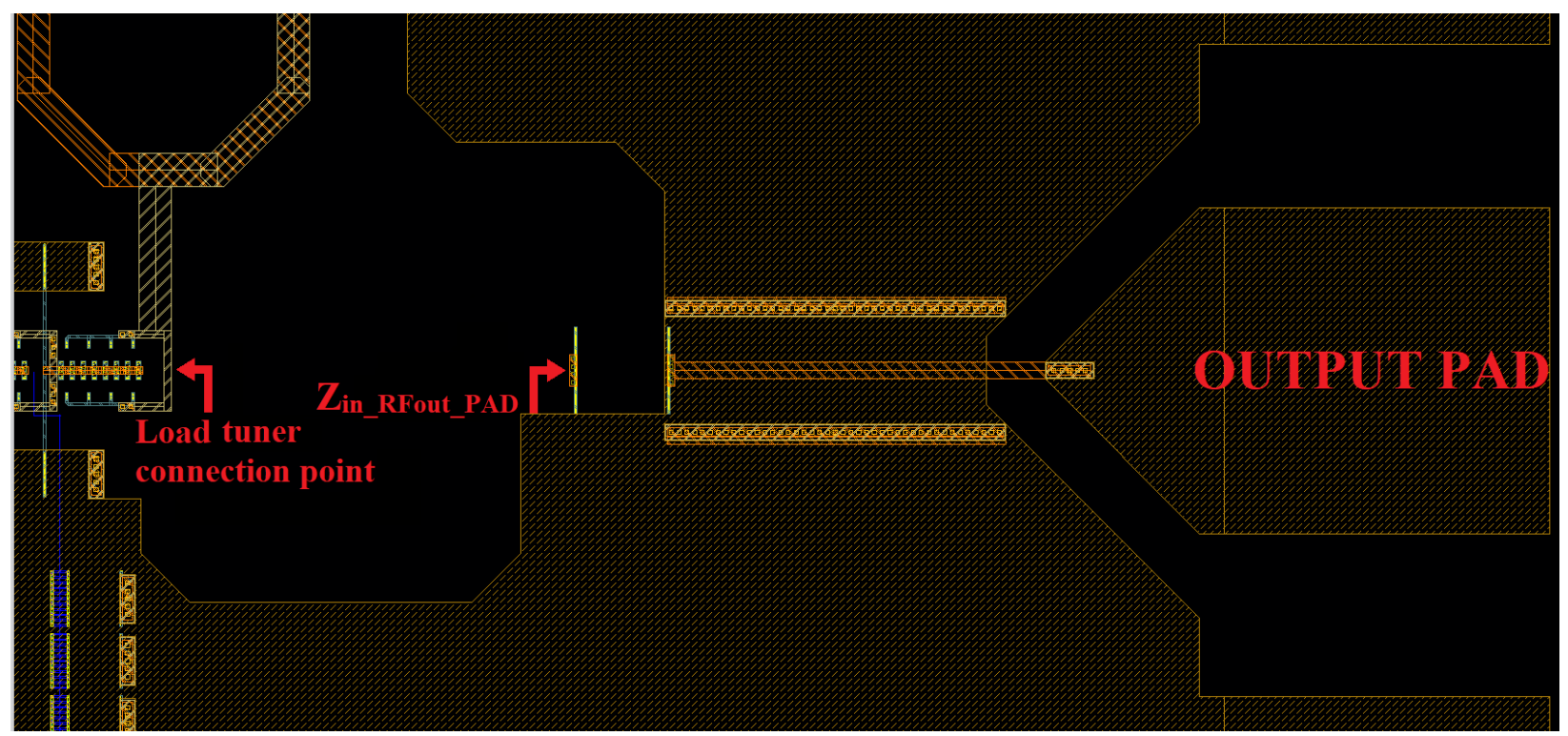

Figure 4.12: Load tuner connection point and the impedance looking towards the RF output pad $\left(Z_{\text {in_RFout_PAD }}\right)$.

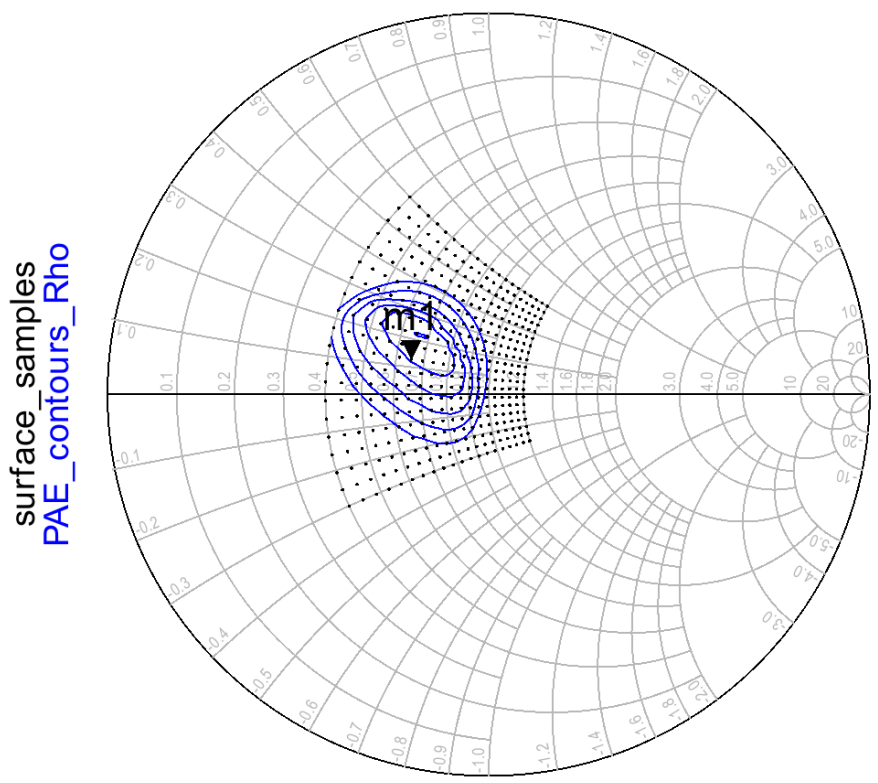

Maximum PAE and contour levels, \%:

32.517

32.000

31.500

31.000

30.500

30.000

\begin{tabular}{|c|}
\hline Zload_at_m1 \\
\hline $32.632+\mathrm{j} 6.316$ \\
\hline
\end{tabular}

Figure 4.13: Sampled load points and PAE contours in $0.5 \%$ steps for $19.5 \mathrm{dBm}$ output power. 
In the same way as was done for the input matching, the impedance seen at the reference plane shown in Figure 4.12 was found with harmonic balance simulations. Then, this impedance was matched to $Z_{\text {opt }}$ using a single-section low-pass $\mathrm{L}$ match. The low-pass matching network was used at the output to filter out the harmonic components that are generated by the nonlinearities of the transistor. Figure 4.14 shows the impedance trajectory from $Z_{\text {in_RFout } P A D}$ to $Z_{\text {opt }}$ where the loaded $Q$ of the matching network was found to be 0.68 .

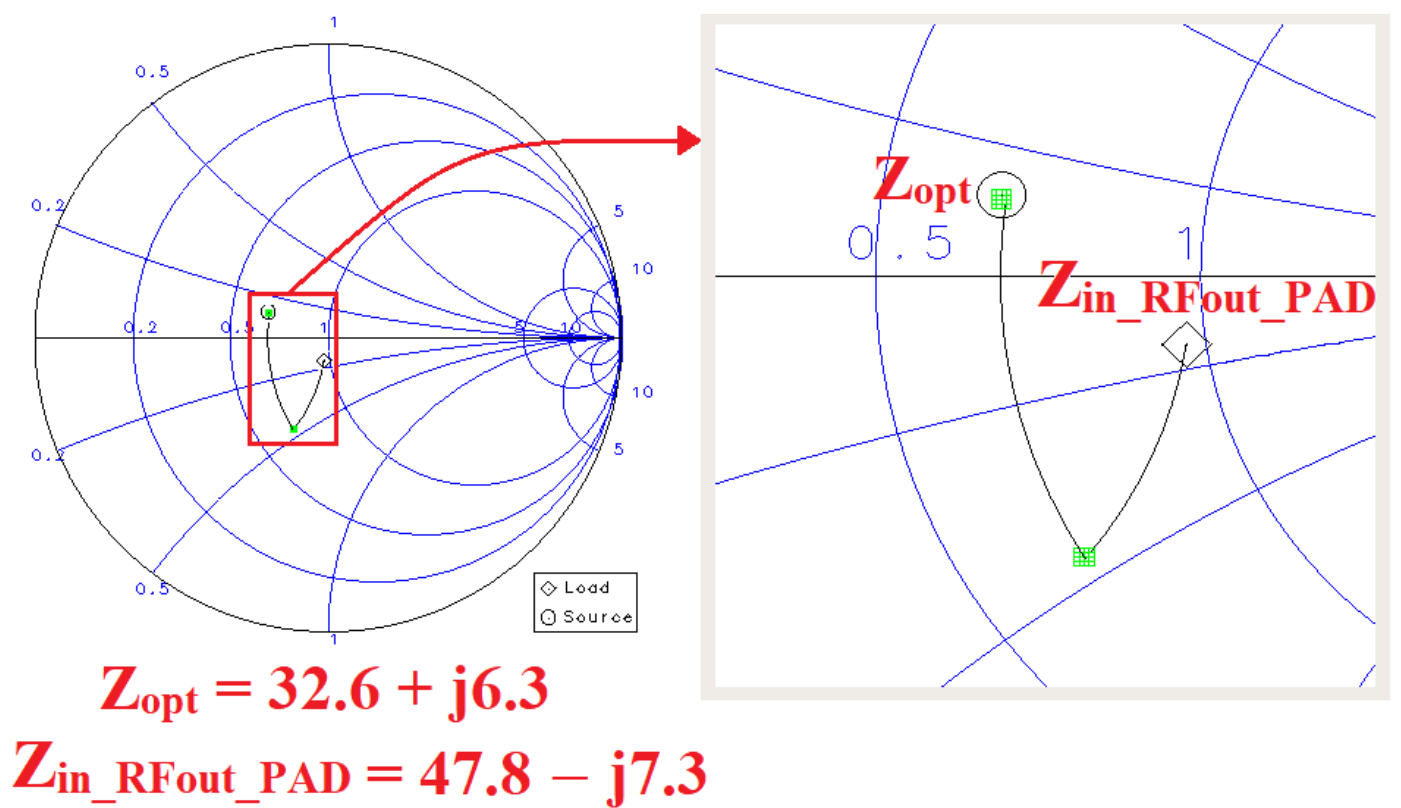

Figure 4.14: Impedance transformation from $Z_{\text {in_RFout } P A D}$ to $Z_{\text {opt }}$ at $28 \mathrm{GHz}$ (loaded $Q=0.68$ ).

Figure 4.15 and Figure 4.16 show the final schematic and layout implementation of the 3-Stack PA, respectively. The implemented PA chip occupies $0.66 \mathrm{~mm}^{2}(950 \mu \mathrm{m}$ by $700 \mu \mathrm{m})$ of die area including the pads. It should be noted that the intermediate node matching was not used as the EM-cosimulation results showed that the phase-misalignments between the drain-source voltages of the individual transistors were minimal. 


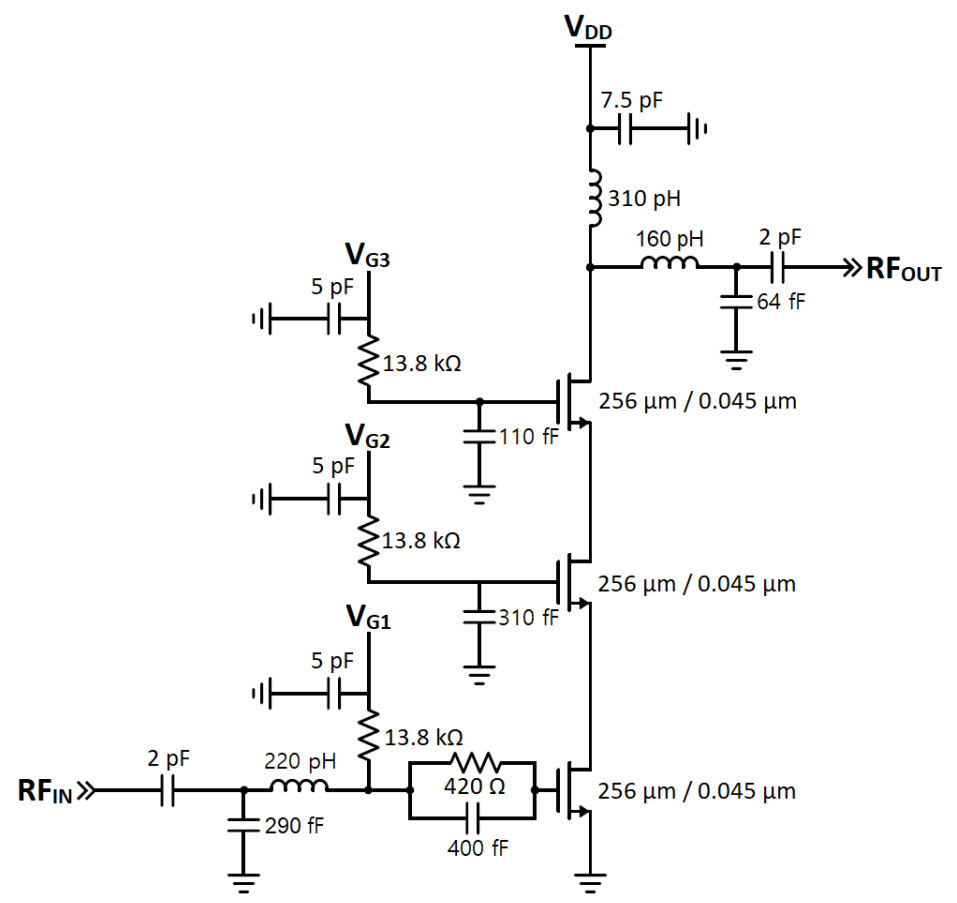

Figure 4.15: Designed single-ended 3-stack PA, $V_{D D}=3.3 \mathrm{~V}, V_{G 1}=0.55 \mathrm{~V}, V_{G 2}=1.65 \mathrm{~V}, V_{G 3}=$ $2.75 \mathrm{~V}$.

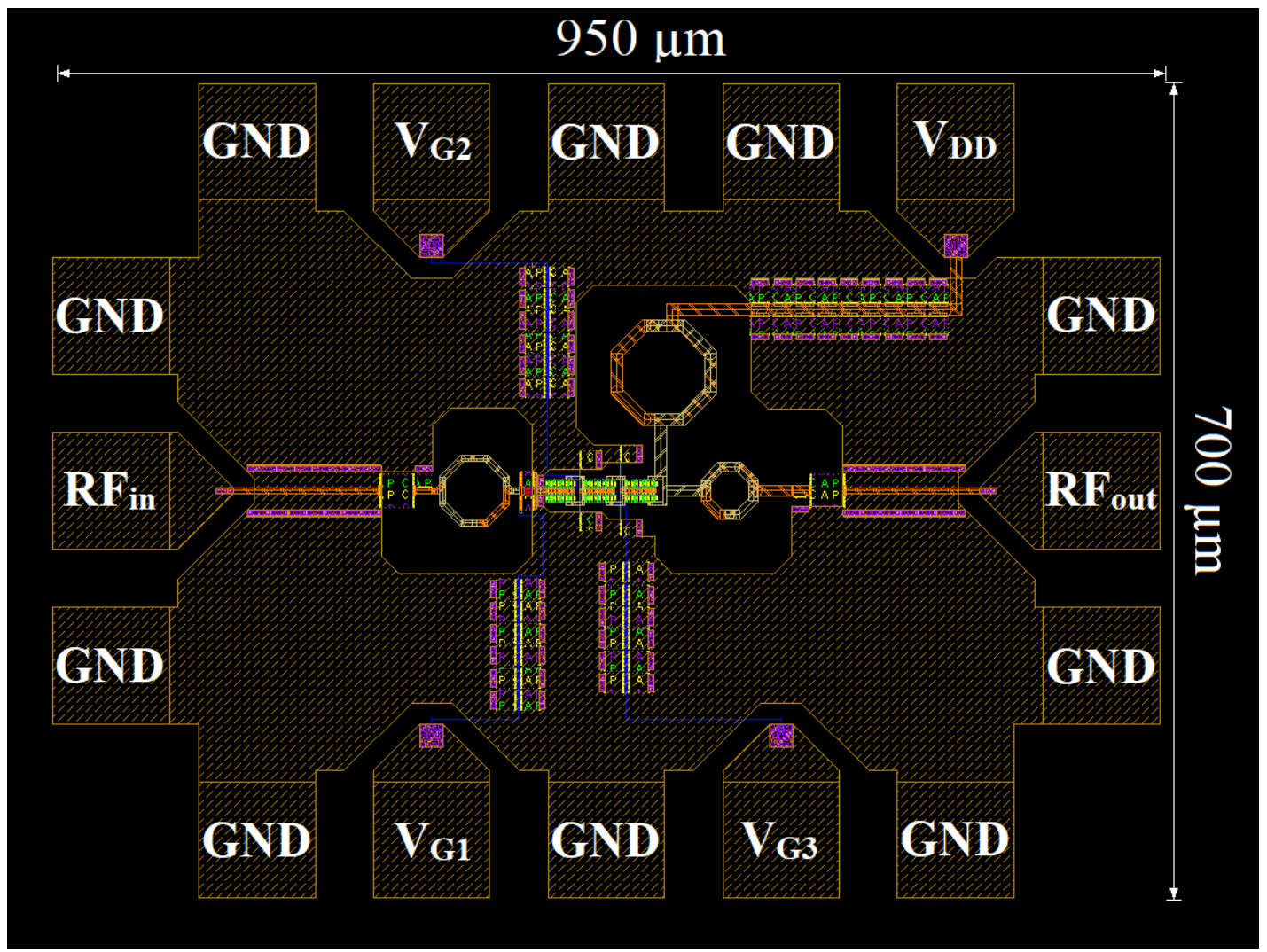

Figure 4.16: Layout implementation of the single-ended 3-stack PA $(950 \mu \mathrm{m} \times 700 \mu \mathrm{m})$. 


\subsection{EM-Cosimulation Results}

Figure 4.17 shows the small-signal S-parameter simulation result of the implemented PA. The PA achieves a maximum small-signal gain of $14.1 \mathrm{~dB}$ at $28 \mathrm{GHz}$ and a 3-dB gain bandwidth of 10.3 GHz (20.8-31.1 GHz), which corresponds to $36 \%$ fractional bandwidth. Furthermore, the PA achieves an input return loss of better than $10 \mathrm{~dB}$ over $27 \mathrm{GHz}$ to $29 \mathrm{GHz}$. Figure 4.18 to Figure 4.22 shows the large-signal performance of the PA under a continuous wave (CW) RF input. At the operating frequency of $28 \mathrm{GHz}$, the PA delivers a saturated output power $P_{\text {sat }}$ of $19.5 \mathrm{dBm}(89 \mathrm{~mW})$ and achieves a peak PAE of $31.8 \%$. It can be seen from Figure 4.21 that at the maximum input drive, the drain-source voltages of the individual transistors are operating within the BVDSS limit of a single device $(\mathrm{BVDSS}=2.2 \mathrm{~V})$ and they are all phase-aligned. Lastly, Figure 4.23 and Figure 4.24 show that the AM-PM variation at the saturated output power is about $1.7^{\circ}$, and the PA is unconditionally stable.

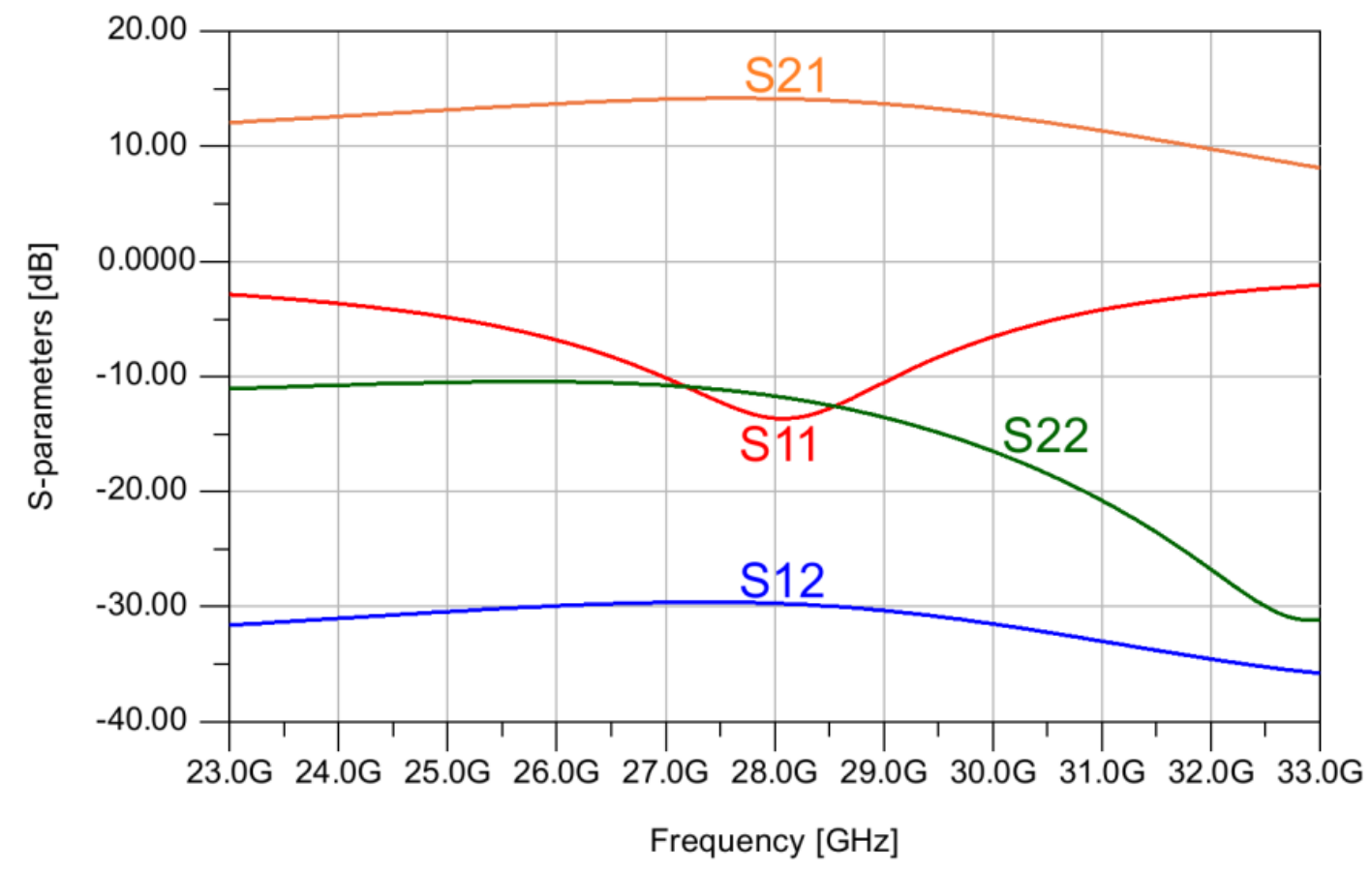

Figure 4.17: S-parameters versus frequency. 


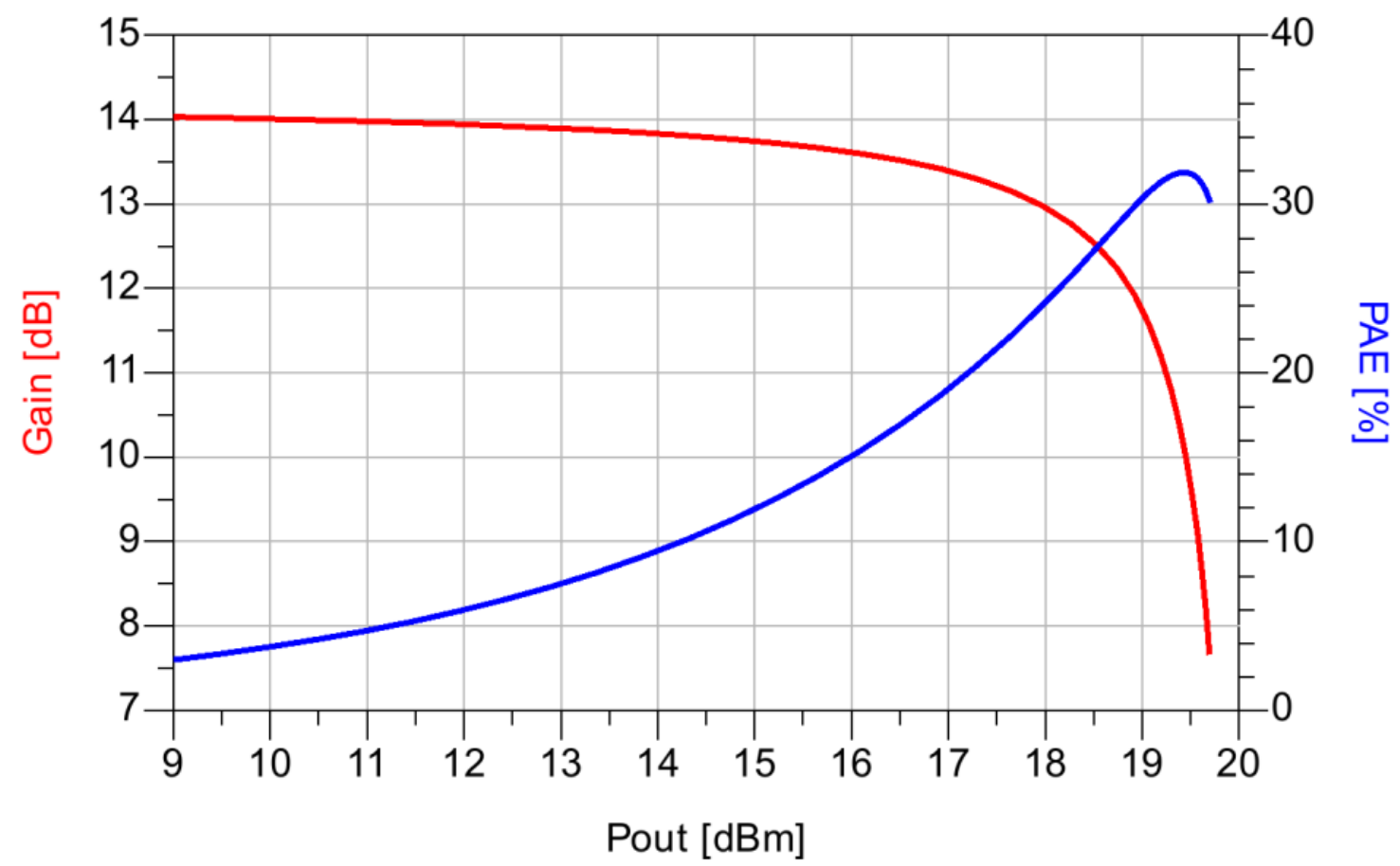

Figure 4.18: Gain and power-added efficiency versus output power at $28 \mathrm{GHz}$.

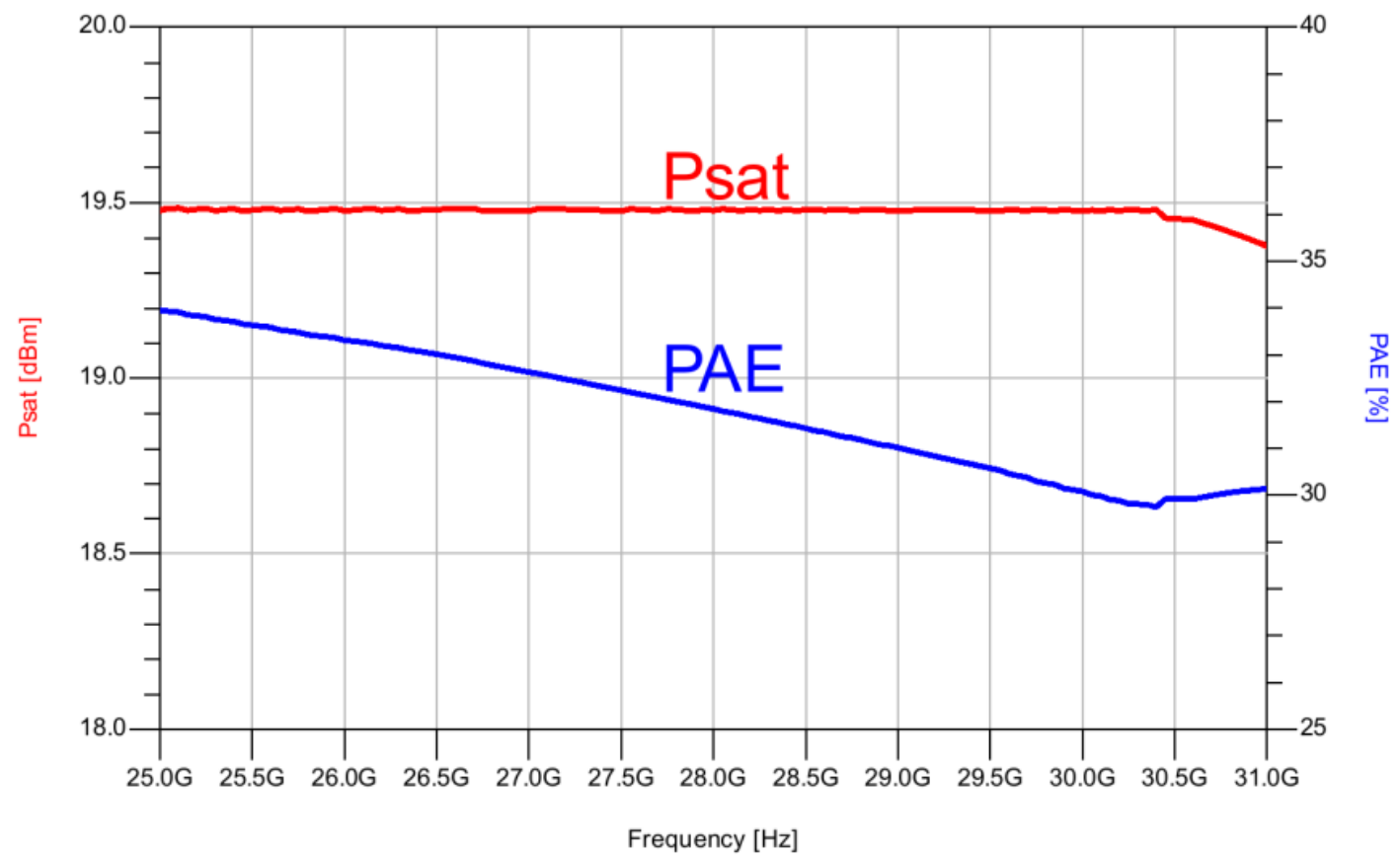

Figure 4.19: Power-added efficiency and saturated output power versus frequency. 


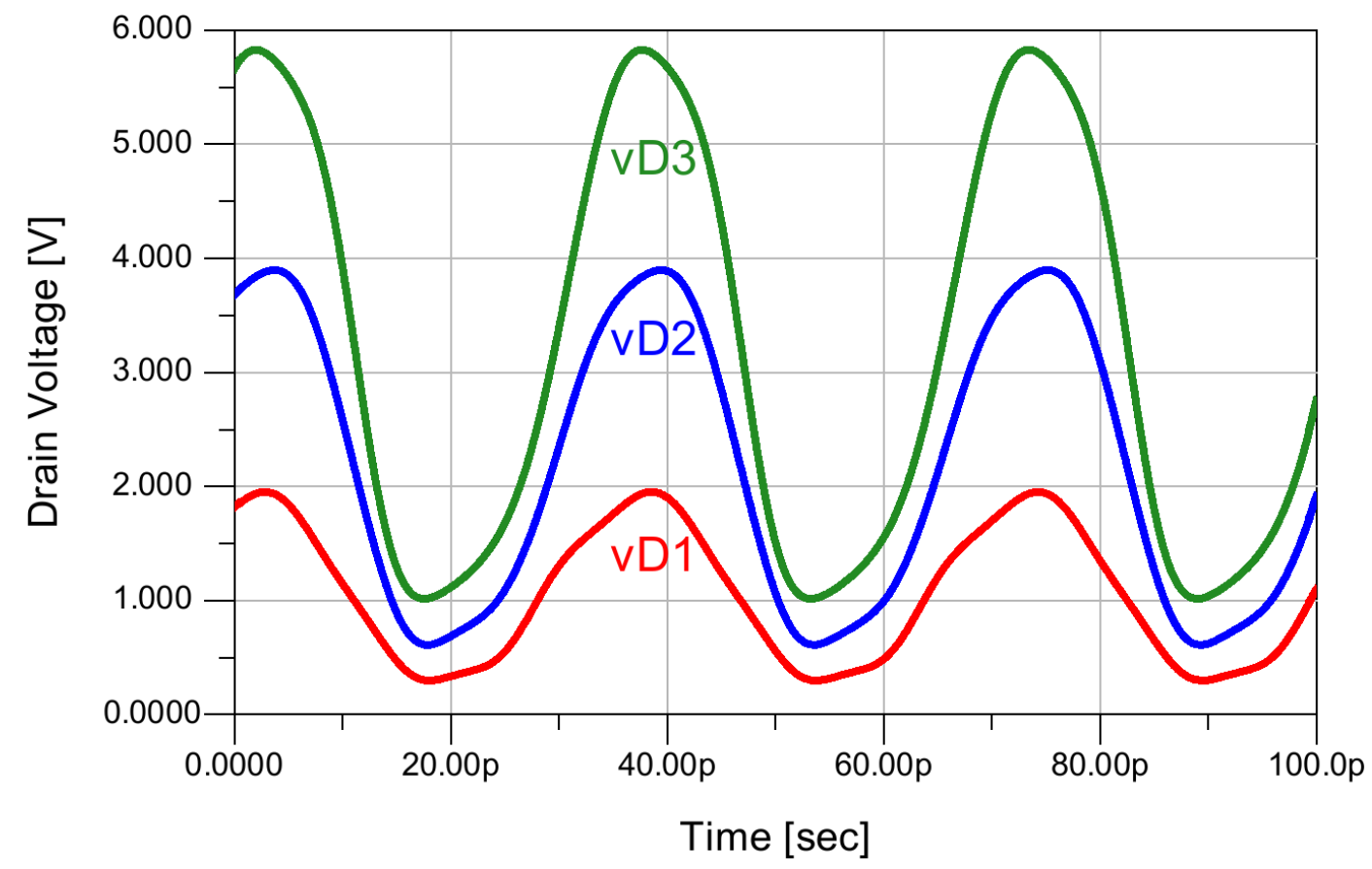

Figure 4.20: Drain voltages versus time.

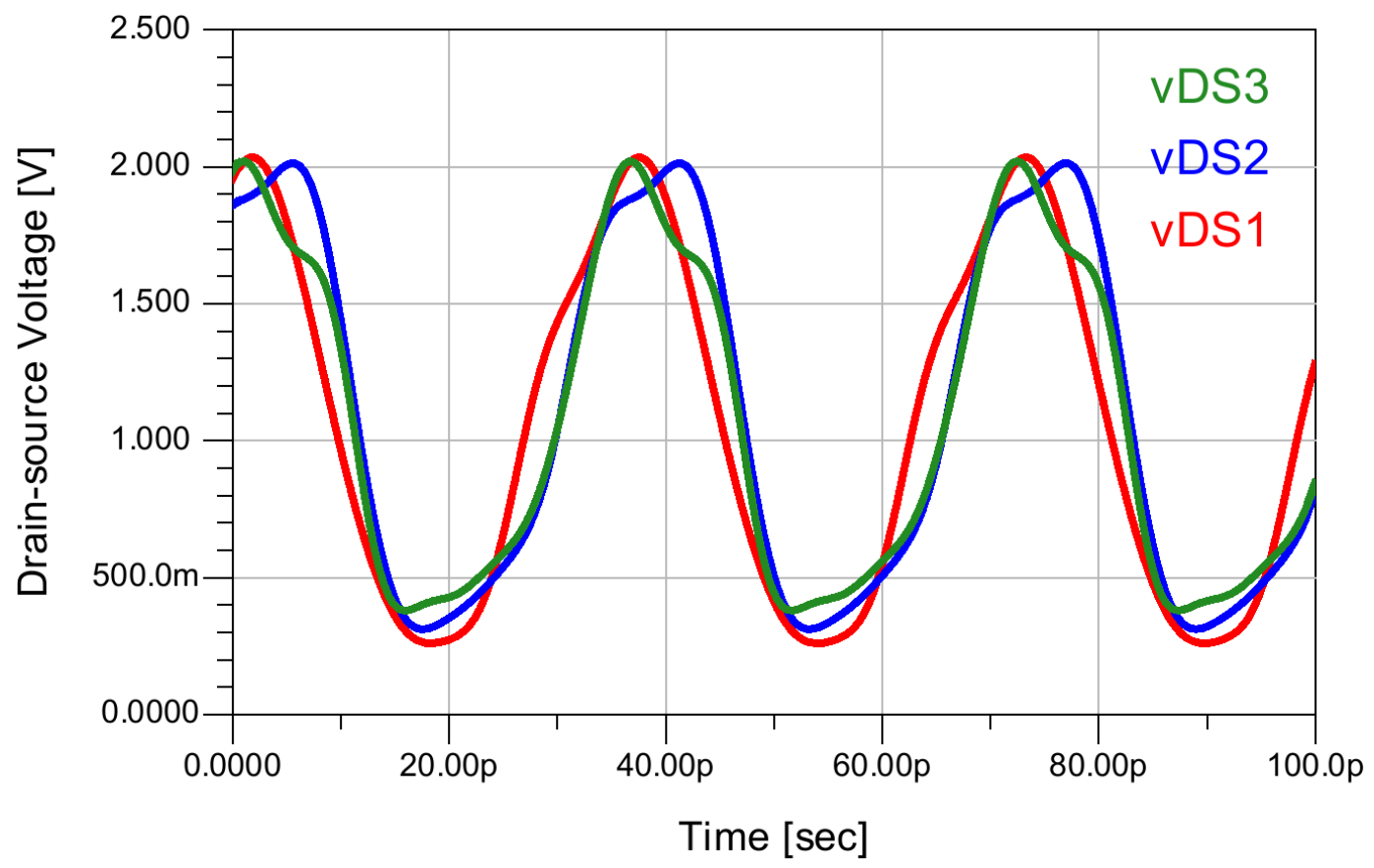

Figure 4.21: Drain-source voltages versus time. 
CHAPTER 4. 28 GHz 3-STACK POWER AMPLIFIER IN 45-nm RF-SOI

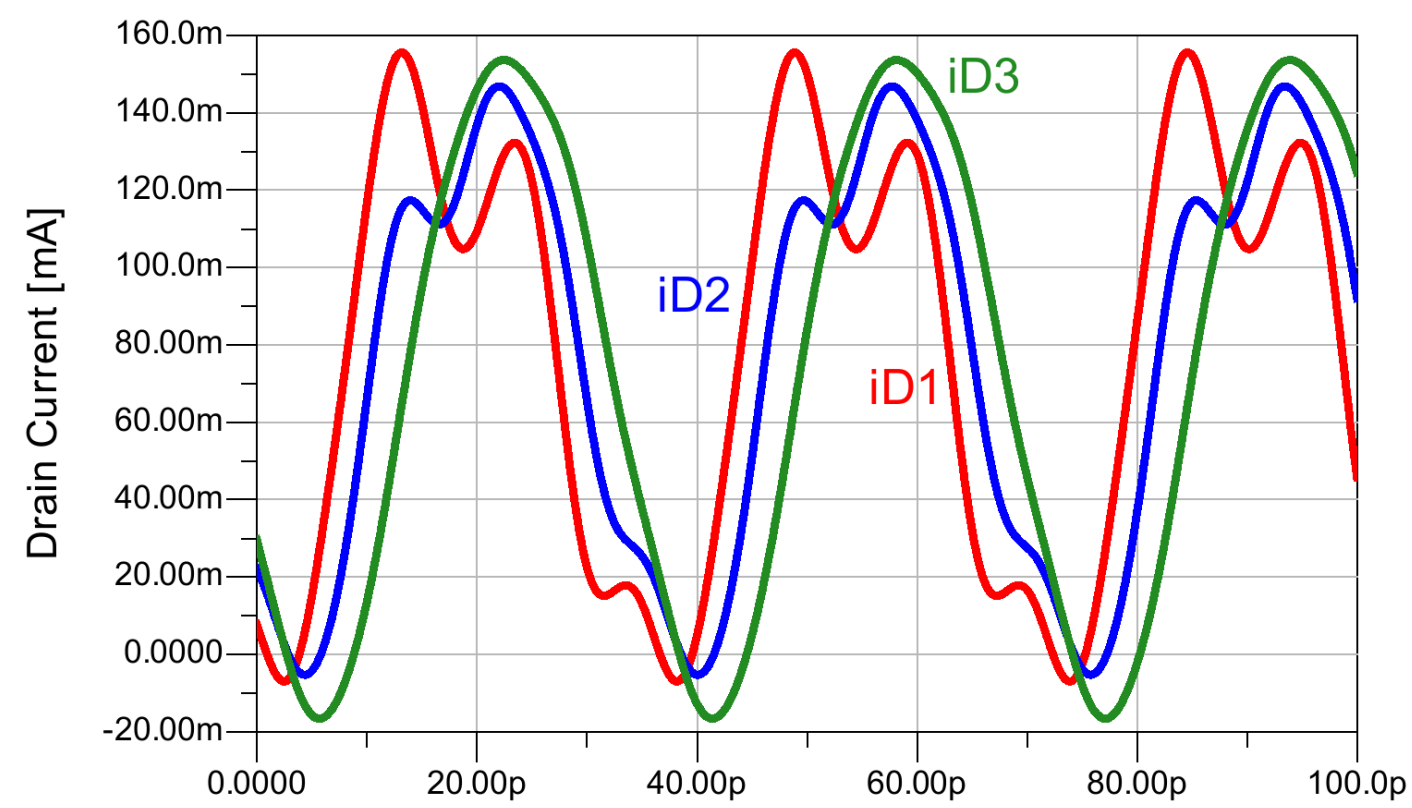

Figure 4.22: Drain currents versus time.

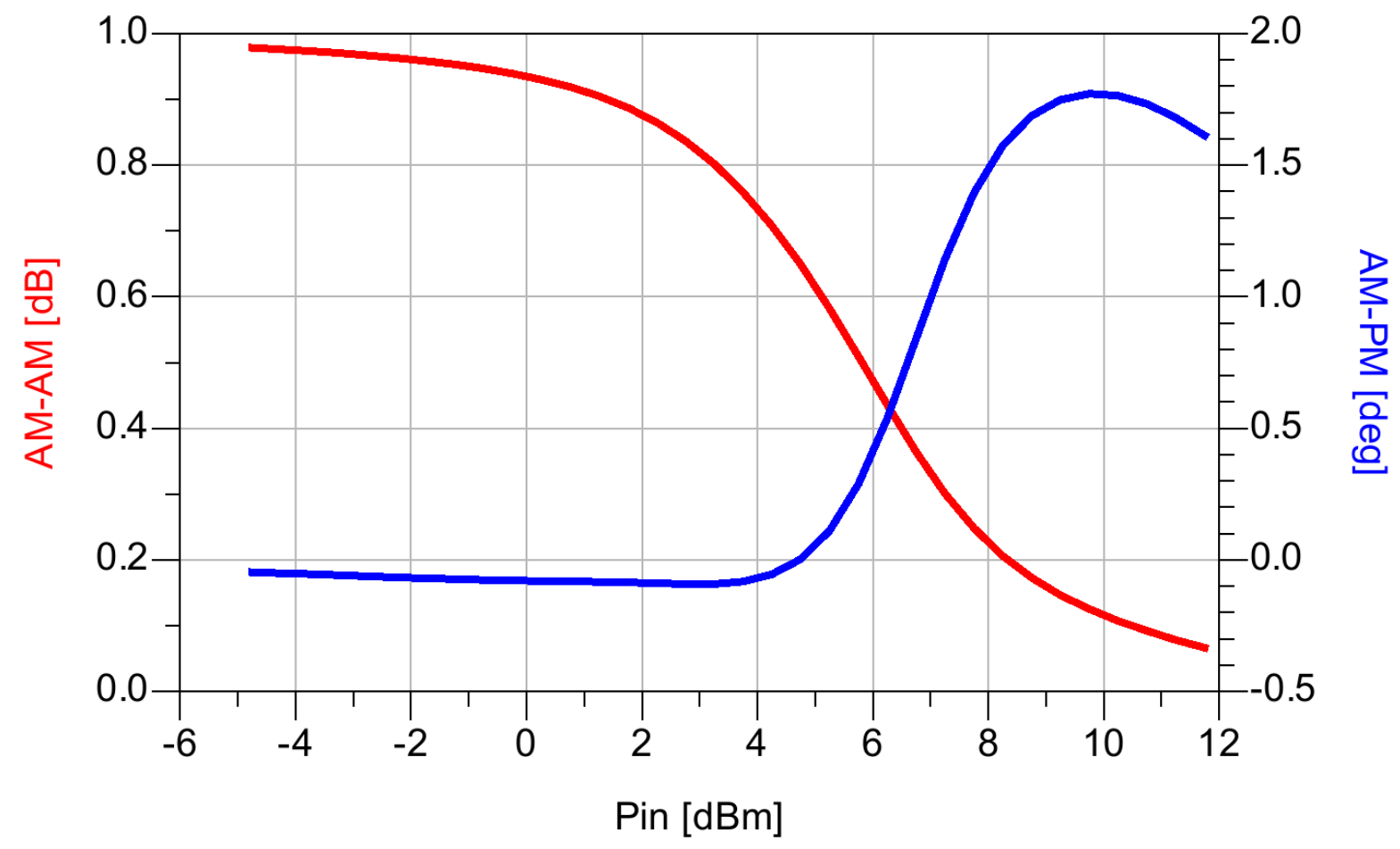

Figure 4.23: $\mathrm{AM}-\mathrm{AM}$ and $\mathrm{AM}-\mathrm{PM}$ conversion at $28 \mathrm{GHz}$. 


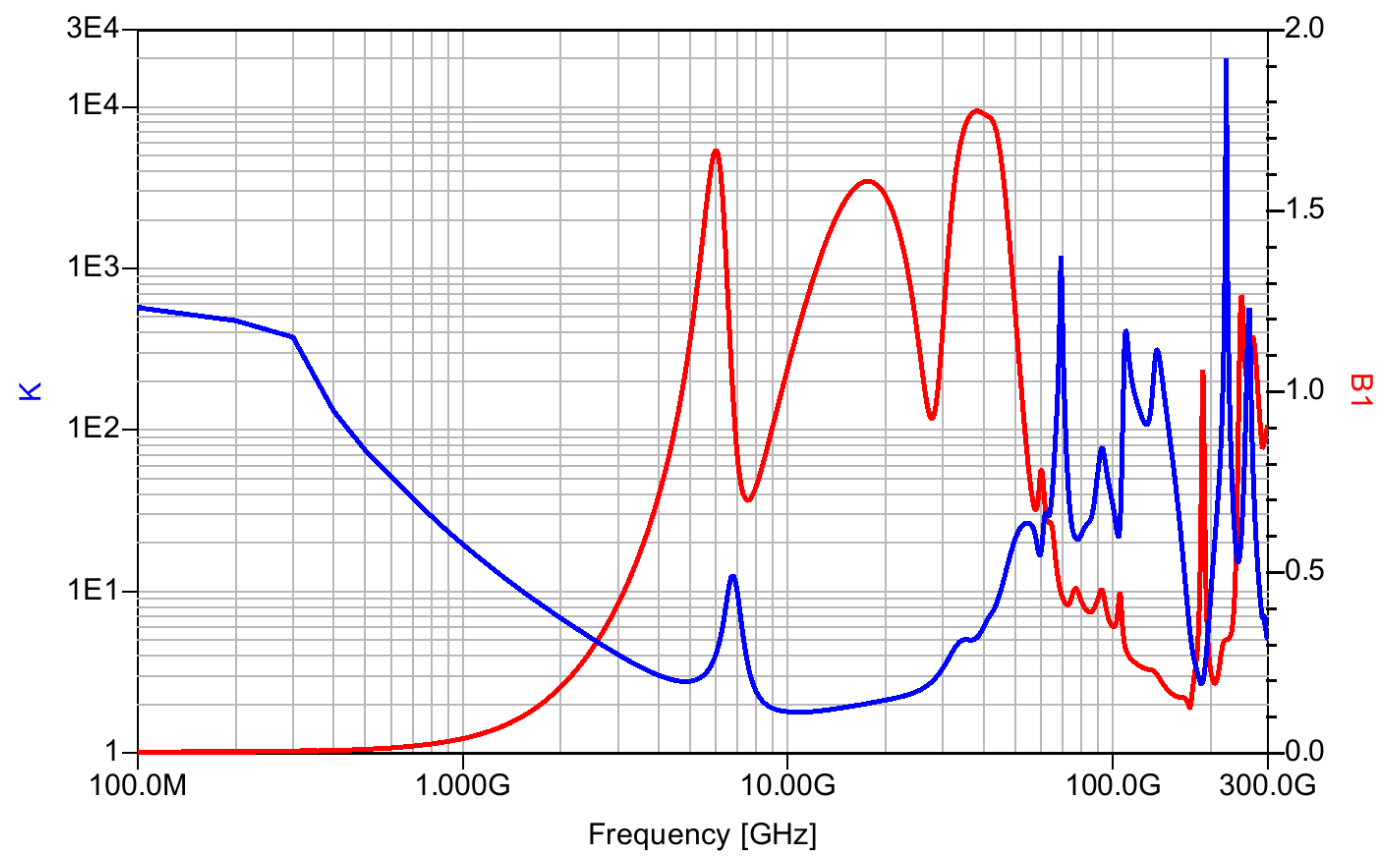

Figure 4.24: Stability factor $\mathrm{K}$ and $\mathrm{B}_{1}$ versus frequency.

The performance of the 3-stack PA is summarized in Table 4.3.

Table 4.3: Performance summary of the 3-stack PA.

\begin{tabular}{|c|c|}
\hline Specification & Achieved \\
\hline Operating Frequency $\left(f_{o}\right)[\mathrm{GHz}]$ & 28 \\
\hline Power Supply Voltage $\left(V_{D D}\right)[\mathrm{V}]$ & 3.3 \\
\hline 1-dB Compression Point $\left(P_{l d B}\right)[\mathrm{dBm}]$ & 18 \\
\hline Saturated Output Power $\left(P_{s a t}\right)[\mathrm{dBm}]$ & 19.5 \\
\hline Gain $[\mathrm{dB}]$ & 14.1 \\
\hline Peak PAE $[\%]$ & 31.8 \\
\hline DC Power Consumption $\left(P_{D C}\right)[\mathrm{W}]$ & 0.25 \\
\hline
\end{tabular}

\subsection{Measurement Setup}

Figure 4.25 shows the measurement setup for the fabricated PA chip. The measurements are to be performed on-wafer (in a $50 \Omega$ environment) using $150 \mu \mathrm{m}$ pitch G-S-G probes and $150 \mu \mathrm{m}$ pitch PPPPP DC probes. In order to shift the measurement reference plane to the device-undertest (DUT), calibration up to the probe tips are required using on-wafer short-open-load-thru (SOLT). 

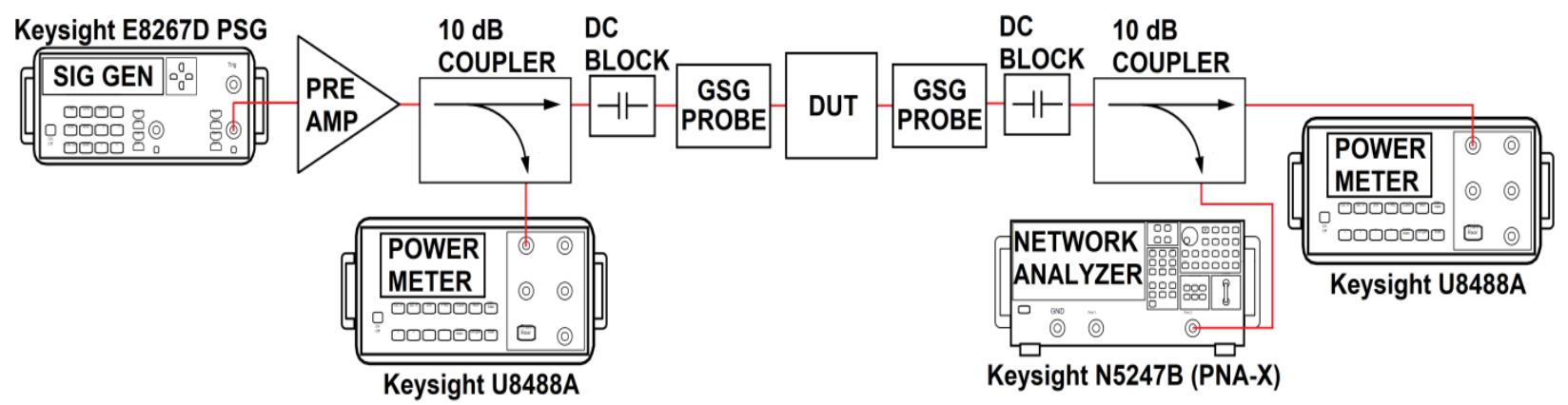

Figure 4.25: Large-signal measurement setup of the fabricated chip [40].

\subsection{Summary}

In this chapter, a fully integrated $28 \mathrm{GHz} 3$-stack PA was implemented using the $256 \mu \mathrm{m}$ power transistor designed in Chapter 3. Transistor stacking was exploited to achieve higher RF output power while overcoming the high knee voltage and low breakdown voltage limitations of the RF-SOI devices. The implemented PA operates from a 3.3V power supply, and under a continuous wave (CW) RF input, it delivers a saturated output power of $19.5 \mathrm{dBm}$ and achieves a peak power-added efficiency (PAE) and a power gain of $31.8 \%$ and $14.1 \mathrm{~dB}$, respectively. The PA chip occupies $0.66 \mathrm{~mm}^{2}(950 \mu \mathrm{m}$ by $700 \mu \mathrm{m})$ of die area including the pads. 


\section{Chapter 5}

\section{Conclusions and Future Work}

\subsection{Conclusions}

mmWave PAs for 5G handsets and small-cell radios are challenged to meet not only the RF performance requirements but also the non-RF performance requirements such as high integration capability, low power consumption, and the capability to offer a large-volume production at a low cost. In this regard, RF-SOI has emerged as an attractive candidate process that has a potential to meet both the RF and non-RF performance requirements of the $5 \mathrm{G}$ mmWave PAs. Consequently, the research work in this thesis aimed to assess the 45-nm RF-SOI process in terms of its feasibility of implementing 5G mmWave PAs for handsets and small-cell radios.

This thesis began by discussing the advantages and limitations of III-V and bulk CMOS processes in relation to $5 \mathrm{G}$ mmWave PAs. The literature review revealed that although the bulk CMOS is capable of achieving the RF output power level required for $5 \mathrm{G}$ mmWave PAs, the 
limited power supply voltage and the lossy on-chip passives forbid achieving the RF performance comparable to that of III-V counterparts.

This thesis then discussed how the shortcomings of the bulk CMOS are remedied in RFSOI to result in an improved RF performance of the circuit. The key features of RF-SOI, such as high $f_{T} / f_{\max }$ PD-SOI devices, high-resistivity substrate, and RF/mmWave-optimized BEOL metal stack were studied. Next, the design and optimization of transistor cell layout in 45-nm RF-SOI process were presented. The impact of layout parasitics (i.e., intrinsic and extrinsic gate resistances and parasitic capacitances) on the $f_{\max }$ of a transistor was discussed and several layout techniques that can be used to improve the $f_{\max }$ of a unit cell were introduced. Then, $256 \mu \mathrm{m}$ power transistor cell layout was implemented using the "zipper" style, which achieved a peak extrinsic $f_{\max }$ of $279 \mathrm{GHz}$.

Lastly, this thesis presented the design and implementation of a fully integrated $28 \mathrm{GHz}$ 3-stack PA. Transistor stacking was exploited to achieve higher RF output power while overcoming the high knee voltage and low breakdown voltage limitations of the RF-SOI devices. Furthermore, in order to reduce the risk of discrepancies between the simulated and measured performance of the PA chip, a layout-driven design method, which incorporates the layout parasitics into the design was adopted. Under a continuous wave RF input, the implemented PA achieved a saturated output power, peak power-added efficiency (PAE), and a power gain of $19.5 \mathrm{dBm}, 31.8 \%, 14.1 \mathrm{~dB}$, respectively at $28 \mathrm{GHz}$. The implemented PA chip occupied a die area of $0.66 \mathrm{~mm}^{2}$ (950 um by $\left.700 \mathrm{um}\right)$.

Unfortunately, due to the recent outbreak of trade war between the United States and China, there was a delay in the tapeout schedule, and the implemented PA chip is currently under fabrication. Also, a mistake was found in the design after the design was submitted for 
fabrication. Nevertheless, the fix for the mistake has been fully incorporated in the PA design presented in this thesis, and the presented EM-cosimulation results are based on the corrected version of the PA.

\subsection{Future Work}

The primary future work is to measure the fabricated PA chip upon its arrival and compare the measured results with the simulated results. Besides the measurement of the chip, the research work presented in this thesis can be further extended in the following area. The 3-stack linear PA implemented in this thesis can be leveraged to design and implement a fully integrated mmWave Doherty PA. The inherent problem with the conventional PAs, such as the one presented in this thesis, is that the peak PAE is achieved only at the saturated output power level, and the PAE declines rapidly from its peak value as the output power is backed-off from the saturated output power level; this implies that the conventional PAs will have low average PAE when they are subjected to modulated signals with high PAPR (as used in the modern wireless communication systems). The low average PAE is problematic as it results in low battery life for batterypowered devices and higher operating costs for the base stations. Furthermore, the low average PAE leads to increased heat-dissipation in the PA, which, in turn, degrades the performance and eventually the life span of the PA; this is particularly concerning for RF-SOI PAs as they already suffer from the self-heating effects. Consequently, maintaining high efficiency over a wide signal dynamic range becomes an important design requirement for modern PAs. The classical Doherty PA is comprised of an input power splitter; two PAs termed the "main" and "auxiliary," and an output combiner; the Doherty PA uses what is called "active load-modulation" to extend the region of high PAE over a wide range of output power (down to $6 \mathrm{~dB}$ back-off for a classical 
Doherty PA). In the bulk CMOS, the full integration approach was deemed rather unpractical as the high losses in the Doherty output combiner diminished the central PAE benefit of the Doherty PA. However, since high $Q$ on-chip passives can be realized in RF-SOI process, it would be worthwhile to investigate what sort of performance can be achieved with a fully integrated Doherty PA implemented in RF-SOI process. 


\section{List of References}

[1] International Telecommunication Union, "5G roadmap: challenges and opportunities ahead," July 2017 [Online]. Available: https://www.itu.int/en/ITU-

D/Conferences/GSR/Documents/GSR2017/IMT2020\%20roadmap\%20GSR17\%20V1\%202 017-06-21.pdf

[2] Skyworks Solutions, Inc., White Paper, "5G in Perspective: A Pragmatic Guide to What's Next,” Mar. 2017. [Online]. Available: https://www.skyworksinc.com/-/media/skyworks/ documents/products/2701-2800/5g-white-paper.pdf

[3] 5G Americas, “5G Spectrum Recommendations,” Aug. 2015. [Online]. Available: https://www.5gamericas.org/wpcontent/uploads/2019/07/4G_Americas_5G_Spectrum_Rec ommendations_White_Paper-2015.pdf

[4] T. S. Rappaport et al., "Millimeter-wave mobile communications for 5G cellular: It will work!" IEEE Access, vol. 1, pp. 335-349, May 2013.

[5] S. Rangan, T. S. Rappaport, E. Erkip, "Millimeter-Wave Cellular Wireless Networks: Potentials and Challenges," Proceedings of the IEEE, vol. 102, no. 3, pp. 366-385, Mar. 2014.

[6] D. Y. C. Lie, J. C. Mayeda, and J. Lopez, "A Short Survey on Recent Highly Efficient cmWave 5G Linear Power Amplifier Design," in Proc. IEEE $60^{\text {th }}$ International Midwest Symposium on Circuits and Systems (MWSCAS), Oct. 2017, pp. 1-4.

[7] D. Y. C. Lie, J. Tsay, T. Hall, T. Nukala, and J. Lopez, "High-Efficiency Silicon RF Power Amplifier Design - Current Status and Future Outlook,” in Proc IEEE International Symposium on Radio-Frequency Integration Technology (RFIT), Aug. 2016, pp. 1-4.

[8] GlobalFoundries, Inc., “45RFSOI: Advanced 45nm RF SOI Technology,” [Online]. Available: https://www.globalfoundries.com/sites/default/files/product-briefs/pb-45rfsoi.pd 
[9] Analog Devices, Inc., "RF Technology For the 5G Millimeter Wave Radio,” Nov. 2016. [Online]. Available: https://www.analog.com/media/en/technical-documentation/whitepapers/RF-Technology-for-the-5G-Millimeter-Wave-Radio.pdf

[10] S. Shakib, J. Dunworth, V. Aparin, and K. Entesari, "mmWave CMOS Power Amplifiers for 5G Cellular Communication," IEEE Communications Magazine, vol. 57, no. 1, pp. 98105, Jan. 2019.

[11] A. M. Niknejad and H. Hashemi, mm-Wave Silicon Technology $60 \mathrm{GHz}$ and Beyond, Springer, 2008.

[12] H. -T. Dabag, B. Hanafi, F. Golcuk, A. Agah, J. F. Buckwalter, and P. M. Asbeck, "Analysis and Design of Stacked-FET Millimeter-Wave Power Amplifiers," IEEE Transactions on Microwave Theory and Techniques, vol. 61, no. 4, pp. 1543-1556, Apr. 2013.

[13] GlobalFoundries, Inc., "RF SOI: Defining the RF-Digital Boundary for 5G," Jan. 2016. [Online]. Available: http://soiconsortium.eu/wpcontent/uploads/2016/01/GLOBALFOUNDRIES_RFSOI_5G_Tokyo_January_2016.pdf

[14] GlobalFoundries, Inc., "Delivering on the Promises of 5G: SOI RF Semiconductor Solutions for the Next Wave of Data," June 2017. [Online]. Available: http://soiconsortium.eu/wp-content/uploads/2017/02/GLOBALFOUNDRIES-SOIConsortium-Tokyo-June-2017-PRabbeni.pdf

[15] GlobalFoundries, Inc., “45nm RFSOI for microwave / mmWave,” Sept. 2015.

[16] K. Ning and J. F. Buckwalter, "A 28-GHz, 18-dBm, 48\% PAE Stacked-FET Power Amplifier with Coupled-Inductor Neutralization in 45-nm SOI CMOS," in 2018 IEEE BiCMOS and Compound Semiconductor Integrated Circuits and Technology Symposium (BCICTS), Oct. 2018, pp. 85-88.

[17] C. Li et al., "A High-Efficiency 5G K/Ka-Band Stacked Power Amplifier in 45nm CMOS SOI process Supporting 9Gb/s 64-QAM Modulation with 22.4\% Average PAE," in 2017 
Texas Symposium on Wireless and Microwave Circuits and Systems (WMCS), Mar. 2017, pp. $1-4$.

[18] N. Rostomyan, M. Ozen, and P. M. Asbeck, "Comparison of pMOS and nMOS $28 \mathrm{GHz}$ High Efficiency Linear Power Amplifier in 45 nm CMOS SOI,” in 2018 IEEE Topical Conference on RF/Microwave Power Amplifiers for Radio and Wireless Applications (PAWR), Jan. 2018. pp. 26-28.

[19] N. Rostomyan, M. Ozen, and P. M. Asbeck, “28 GHz Doherty Power Amplifier in CMOS SOI With 28\% Back-Off PAE," IEEE Microwave and Wireless Components Letters, vol. 28, no. 5, pp. 446-448, May 2018.

[20] M. Ozen, N. Rostomyan, K. Aufinger, and C. Fager, "Efficient millimeter wave Doherty PA design based on a low-loss combiner synthesis technique," IEEE Microwave Wireless Component Letters, vol. 27, no. 12, pp. 1143-1145, Dec. 2017.

[21] J. A. Jayamon, J. F. Buckwalter, and P. M. Asbeck, "Multigate-Cell Stacked FET Design for Millimeter-Wave CMOS Power Amplifiers," IEEE Journal of Solid-State Circuits, vol. 51, no. 9, pp. 2027-2039, Sept. 2016.

[22] P. Reynaert, Y. Cao, M. Vigilante, and P. Indirayanti, "Doherty techniques for 5G RF and mm-wave Power Amplifiers," in Proc. 2016 International Symposium on VLSI Technology, Systems, and Application (VLSI-TSA), Apr. 2016, pp. 1-2.

[23] J. W. Rogers and C. Plett, Radio Frequency Integrated Circuit Design, $2^{\text {nd }}$ ed. Artech House, 2003.

[24] G. Hueber and A. M. Niknejad, Millimeter-Wave Circuits for $5 G$ and Radar, Cambridge University Press, 2019.

[25] M. K. Kazimierczuk, RF Power Amplifiers, Wiley, 2008.

[26] B. Razavi, RF Microelectronics, $2^{\text {nd }}$ ed. Prentice Hall, 2011.

[27] G. G. Shahidi, "SOI technology for the GHz era," IBM Journal of Research and Development, vol 46, no. 2/3, pp. 121-131, Mar. 2002. 
[28] J. -P. Raskin, "SOI technology: an opportunity for RF designers?," in Proc. 2014 International Symposium on VLSI Technology, Systems, and Application (VLSI-TSA), Apr. 2014, pp. 1-2.

[29] GlobalFoundries, Inc., “45nm RFSOI for microwave / mmWave,” Sept. 2015

[30] B. M. Tenbroek, M. S. L. Lee, W. Redman-White, R. J. T. Bunyan, and M. Uren, "SelfHeating Effects in SOI MOSFET's and Their Measurement by Small Signal Conductance Techniques," IEEE Transactions on Electron Devices, vol. 43, no. 12, pp. 2240-2248, Dec. 1996.

[31] W. Wei, O. K. Jensen, and J.H. Mikkelsen, "Self-heating and Memory Effects in RF Power Amplifiers Explained Through Electro-Thermal Modeling," NORCHIP, pp. 1-4, Nov. 2013.

[32] SOI Industry Consortium, "Fully Depleted (FD) vs. Partially Depleted (PD) SOI," May 2008. [Online]. Available: https://soiconsortium.org/2008/05/14/fully-depleted-fd-vspartially-depleted-pd-soi/

[33] I. D. Robertson and S. Lucyszyn, RFIC and MMIC Design and Technology, The Institution of Engineering and Technology, 2001.

[34] O. Inac, M. Uzunkol, and G. M. Rebeiz, "45-nm CMOS SOI Technology Characterization for Millimeter-Wave Applications," IEEE Transactions on Microwave Theory and Techniques, vol. 62, no. 6, pp. 1301-1311, June 2014.

[35] U. Gogineni, J. A. del Alamo, and C. Putnam, "RF Power Potential of $45 \mathrm{~nm}$ CMOS technology," in 2010 Topical Meeting on Silicon Monolithic Integrated Circuits in RF Systems (SiRF), Jan. 2010, pp. 204-207.

[36] S. Lee, B. Jagannathan, S. Narasimha, A. Chou, N. Zamdmer, J. Johnson, R. Williams, L. Wagner, J. Kim, J. O. Plouchart, J. Pekarik, S. Springer, and G. Freeman, "Record RF performance of 45-nm SOI CMOS Technology," in 2007 IEEE International Electron Devices Meeting, Dec. 2007, pp. 255-258.

[37] J. Zhan, "Millimeter-wave transistor device," U.S. Patent US8653564B1, Feb. 2014. 
[38] B. Heydari, M. Bohsali, E. Adabi, and A. M. Niknejad, "Millimeter-Wave Devices and Circuit Blocks up to $104 \mathrm{GHz}$ in $90 \mathrm{~nm}$ CMOS," IEEE Journal of Solid-State Circuits, vol. 42, no. 12, pp. 2893-2903, Dec. 2007.

[39] S. Pornpromlikit, H. -T. Dabag, B. Hanafi, J. Kim, L. E. Larson, J. F. Buckwalter, and P. M. Asbeck, “A Q-Band Amplifier Implemented with Stacked 45-nm CMOS FETs,” in 2011 IEEE Compound Semiconductor Integrated Circuits Symposium (CSICS), Oct. 2011, pp. 14.

[40] H. -T. Dabag, B. Hanafi, F. Golcuk, A. Agah, J. F. Buckwalter, and P. M. Asbeck, “Analysis and Design of Stacked-FET Millimeter-Wave Power Amplifiers," IEEE Transactions on Microwave Theory and Techniques, vol. 61, no. 4, pp. 1543-1556, Apr. 2013.

[41] J. Cui, S. Helmi, Y. Tang, and S. Mohammadi, "Stacking the Deck for Efficiency: RF-to Millimeter-Wave Stacked CMOS SOI Power Amplifiers," IEEE Microwave Magazine, vol. 17, no. 12, pp. 55-69, Dec. 2016.

[42] C. Li, A. Kumar, X. Tian, N. Cahoon, M. Boenke, D. Wang, A. Joseph, H. Wang, G. Rebeiz, "Ka Band FEM Design Comparison with 45nm RFSOI CMOS and High Performance SiGe BiCMOS," in 2018 IEEE International Conference on Solid-State and Integrated Circuit Technology (ICSICT), Nov. 2018, pp. 1-4.

[43] E. Takeda, N. Suzuki, T. Hagiwara, "Device Performance Degradation Due to Hot-Carrier Injection at Energies below the $\mathrm{Si}_{-} \mathrm{SiO}_{2}$ Energy Barrier," in 1983 International Electron Devices Meeting, Dec. 1983, pp. 1-4.

[44] International Telecommunication Union, "Recommendation ITU-R M.2083-0: IMT Vision - Framework overall objectives of the future development of IMT for 2020 and beyond," Sep. 2015 [Online]. Available: https://www.itu.int/dms_pubrec/itu-r/rec/m/R-REC-M.20830-201509-I!!PDF-E.pdf

[45] SOI Industry Consortium, "Questions and Answers on Fully Depleted SOI Technology (for next generation CMOS nodes)," Apr. 2010 [Online]. Available: https://www.soitec.com/media/documents/8/file/soiconsortium_fdsoi_qa.pdf 